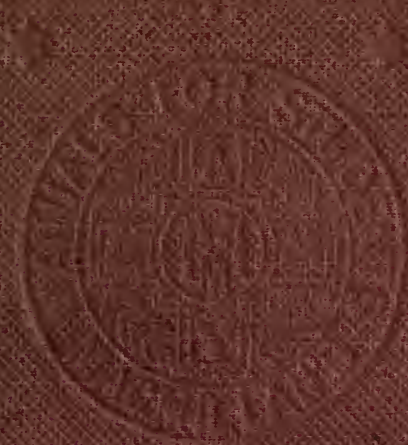



Digitized by the Internet Archive in 2007 with funding from Microsoft Corporation 




\section{ELEMENTARY PRACTICAL BACTERIOLOGY}


2. 


\section{A COURSE \\ OF \\ ELEMENTARY PRACTICAL \\ BACTERIOLOGY \\ INCLUDING \\ BACTERIOLOGICAL ANALYSIS AND \\ CHEMISTRY}

BY

A. A. KANTHACK, M.D., M.R.C.P.

LECTURER ON PATHOLOGY AND BACTERIOLOGY, AND CURATOR OF THE MUSEUM, ST. BARTHOLOMEW'S HOSPITAL

AND

J. H. DRYSDALE, M.B., M.R.C.P.

CASUALTY PHYSICIAN, ST. BARTHOLOMEW'S HOSPITAL; LATE DEMONSTRATOR OF BACTERIOLOGX, UNIVERSITY COLLEGE, LIVERPOOL

\section{R \\ Zlonon}

MACMILLAN AND CO.

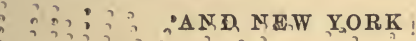

$1895, \therefore \circ$ 



\section{PREFACE}

BACTERIology is an essential branch of Pathology, of such importance that it is gradually becoming a necessary, if not a compulsory, element in the study of Medicine. As a matter of fact, most Faculties and Universities granting a Diploma or Degree in Public Health demand three months' practical instruction in Bacteriology. It is only by patient laboratory work that Bacteriology can be taught in such a manner as to serve any useful purpose.

One of the chief difficulties in the practical instruction which has presented itself to us has been the want of a suitable handbook for the laboratory. The student has felt this want as much as his teachers. He cannot be expected to dig the necessary information out of large and voluminous text-books, which, moreover, are often deficient in all practical details. At St. Bartholomew's Hospital we have been in the habit of giving out slips with full directions about the work to be done from day to day. This method entails a certain amount of disagreeable 
trouble on the part of the lecturer, and finds only partial favour with the student.

We have therefore ventured to collect and put together the slips and notes in the form and shape of a small handbook, which, though originally intended for use at the laboratories of St. Bartholomew's Hospital, we hope may supply a want felt elsewhere. The work is divided into Lessons, and the book is arranged in three parts, of which Parts I. and II. (Elementary Bacteriology and Bacteriological Analysis) encompass three months' work, as required for the Diploma of Public Health at London and Cambridge. Naturally, we have adhered to our own system of teaching, which represents that used for some years at the Pathological Laboratories of Cambridge University and St. Bartholomew's Hospital. A few explanatory remarks, therefore, appear necessary.

At St. Bartholomew's Hospital we invariably begin with an Elementary Course (Part I.), which extends over five to six weeks (three lessons a week). All media are supplied, so that the simpler methods of work, inoculations, cultivations, staining, etc., may be quickly acquired and the student may become acquainted with the general principles. The rest of the term is then devoted to Bacteriological Analysis (Part II.), which includes the preparation of the various media, and the analysis of air, water, food, etc., the examination of filters, disinfectants, etc. As far as possible we have given full directions, but these Lessons are not intended to supplant the demonstrator, nor do 
they pretend to cover the whole ground of Practical Bacteriology. In three months it is impossible to teach or to learn much. Hence we have attempted to make the Lessons as representative as possible, and to introduce the student to the more important analytical studies and problems. Parts I. and II. are especially arranged to suit the requirements of candidates for one or other Diploma in Public Health.

We have always described methods which we use ourselves, and in all cases we have given frankly and without reserve what we consider to be the quickest or the best method. Every laboratory has its own ways and means, its "short cuts," and we might almost say its "secrets." We have divulged all our own, not because we flatter ourselves that there are no better methods, but because we have found from practical experience that most methods depend in their execution on some small detail which is frequently withheld from the beginner. These so-called "laboratory tips," which are acquired through experience and practice, are often omitted in descriptions of special methods, because they seem unimportant, or possibly too precious. Methods which require acquaintance with glass-blowing, or which recommend themselves readily enough to old hands but seem unsuited to the inexperienced, have been purposely left out.

We confess at once that the methods described in these Lessons for the most part are not our own. They have been carried away from the various laboratories in which 
it has been our privilege to work. Often, no doubt, they have been somewhat modified or rearranged for the purpose of instruction. We feel, however, that we must give expression to our indebtedness to Dr. E. Klein for many hints and methods which were legitimately acquired by us from his laboratory. Many, if not most, of the useful methods of Bacteriological Analysis current in this country have emanated thence, and we therefore do not hesitate to acknowledge gratefully all the assistance which consciously or unconsciously we have derived from that liberal source.

The third part of this little book forms, so to speak, an appendix to the two previous parts, and comprises an introduction to Bacteriological Chemistry for those who are desirous of devoting some of their time to advanced investigation and research work. Bacteriological Chemistry is yet a young and unfledged science, even more unsatisfactory than Physiological Chemistry. We have therefore selected a few points in the shape of exercises which are either of classical importance or essential for research.

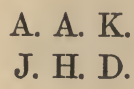

LoNDon, 31st December 1894. 


\section{CONTENTS}

\section{PART I \\ GENERAL BACTERIOLOGY}

\section{LESSON I}

Methods of Inoculation-

PAGE

(1) Agar-Agar Streak Cultures . . . . . . 3

(2) Action of Sunlight on the Bacillus pyocyaneus . . 4

(3) Action of Sunlight on the Bacillus prodigiosus . . 4

(4) Action of Oxygen on the Bacillus prodigiosus . . 5

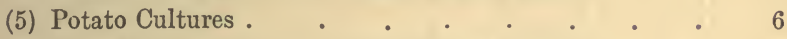

(6) Agar-Agar Cultures of the Bacillus fluorescens . $\quad 6$

(7) Gelatine Streak Cultures . . . . . 6

(9) $\}$ Gelatine Stab Cultures • . . . . . . 6,7

\section{LESSON II}

TyPes of Micro-organisms .

Staining of Mrcro-organisms with Simple Basic Aniline DYes-

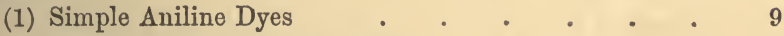

(2) Preparation of Cover-glass Films . • • • . 9

(3) Staining of Films . • • • • . 10 


\section{LESSON III}

Staining of Micro-organisms (continued)-

PAGE

Löffler's Methylene-Blue .

Examination of Moulds-

Aspergillus niger

Hanging Drop Cultures-

(a) Streptococcus pyogenes

(b) Bacillus filamentosus .

Examination of Bacteria in a. Hanging Drop-

Motility of Micro-organisms

\section{LESSON IV}

Staining of Hanging Drop Cultures-

(a) Löffler's Methylene-Blue . .

(b) Gram's Method : . . . . . . 17-19

Gram's Method . . . . . . . . . 17-19

Staining of Pus-

(a) Löffler's Methylene-Blue . . . . . . 20

(b) Gram's Method . . . . . . . . 20

Inoculations with the Bacillus ANthracis-

Potato; Broth ; Agar-Agar ; Gelatine . . . . 21

Curduing Ferment . . . . . . . . . . 21

\section{LESSON V}

Bacillus ANthracis (continued)-

Mode of Growth on Various Media . . . . . 22

Hanging Drop Cultures . . . . . . . 22

Asporogenous Cultures-

(a) Carbolic Acid Broth . . . . . . . . 22

(b) Heat . . . . . . . . . . 23 
Acid Formation by Virulent and Attenuated Bacilli. 23 Methods of Attendation . . . . . . . 23

Staining of the Bacillus Anthracis-

(a) Löffler's Methylene-Blue . . • • . . 23

(b) Gentian-Violet . . . . . . . . 23

(c) Gram's Method . . . . . . . . 24

\section{LESSON VI}

Bacillus Anthracis (continued)-

Hanging Drop Cultures (Staining) • • • • • 25

Asporogenous Cultures (Staining) • . • . . 25

Impression Specimens - • • • • • 26

Examination of Fresh Tissues by Means of Films-

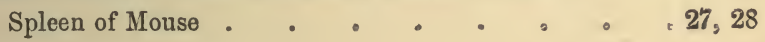

\section{LESSON VII}

Bacrllus Anthracrs (continued)-

Plate Culture Method . . . . . . . 29, 30

Staining of Spores . . . . . . . . 30-32

Ehrlich's, Löffler's, or Ziehl's Fuchsine • . • 30-32

Separation of Hay Bacillus out of Hax Infusion • 33

\section{LESSON VIII}

Bacillus Anthracis (concluded)-

Impression Specimens (Gelatine Plates) . . . . 34

Staining of Tissues in Frozen Sections-

(a) Löfller's Methylene-Blue . . . . . 35

(b) Methylene-Blue and Eosine . . . . . 35, 36

(c) Methylene-Blue and Picrocarmine . . . 36

(d) Gram's Method . . . . . . . . . . . 37 


\section{Cholera-}

Inoculation of Gelatine, Agar-Agar, and Broth with Various Vibrios . 37,38 Staining of Flagella (Van Ermengem)-

(a) Bacillus of Typhoid Fever . . . . . . 38, 39

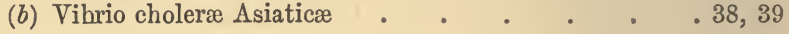

\section{LESSON IX}

Cholera (continued)-

Examination of Vibriones choleræ Asiaticæ, Finkler-Prior, and Metchnicovi in Cultures . . . . . 40

Staining of Vibrios-

(a) Aniline Gentian-Violet . . . . • . 40

(b) Carbol Fuchsine . . . . . . . 40

SPIRILlum RUBRUM-

(a) Staining . . . . . . . . . . . 41

(b) Gelatine Stab Cultures . . . . . . . 41

Hanging Drop Cultures of Vibrio choleræ Asiaticæ . 41

Cholera Vibrios in the Animal Body-

(a) Peritoneal Fluid of Guinea-Pig . . . . . 41

(b) Separation of Vibrios by Plate Cultures . . . 42, 43

Action of Sunlight on the Vibrio Cholerze Asiaticze • 43

\section{LESSON X}

Cholera (concluded)-

Examination of Plates . . . . . . . 44

Impression Specimens . . . . . . . 44

Varieties of Choleraic Vibrios . . . . . . . 44,45 
Actinomycosis-

PAGE

Cladothrix nivea $\quad$. $\quad$. $\quad$. . . . 45

Celloidin Sections of Actinomycosis . • • . . . 45, 46

Mycetoma . . . . . . . . . 46

LESSON XI

Pyogenic Cocci一

Cultures in Broth, Agar-Agar, and Gelatine . . . 47, 48

Hanging Drop Cultures . . . . . . . 48

Staining of Cultures . . . . . . . 48

Staining of Carbolised Pus . . . . . . 48, 49

Staining of Fresh Pus . . . . . . 49

\section{LESSON XII}

Pyogenic Cocci (concluded)-

Staining of Hanging Drops . . . . . 50

Staining in Frozen Sections-

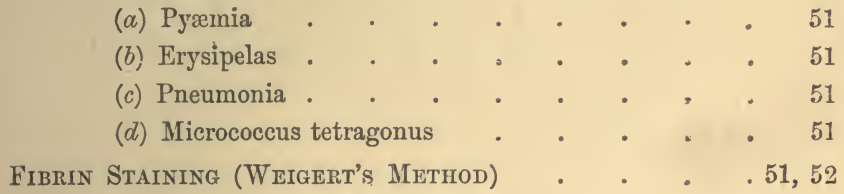

\section{LESSON XIII}

TYPHOID FEVER-

Cultures of the Bacillus of Typhoid Fever. . . . . 53, 54

Cultures of the Bacterium coli commune . . . . . .53, 54

Shake Cultures . . . . . . . . 54

Varieties of the Bacterium coli commune . . . . . 54, 55

Typhoid Spleen (Frozen Sections) . . . . . 55 
Pneumococcus : Staining of Capsule . . . . 55

Staining of Carbolised Pneumonic Sputum . . . 56

Diphtheria-

Cultures of Diphtheria Bacillus _ . . . . . . . . 56,57

Staining of Cover-glass Films . . . . . . 57

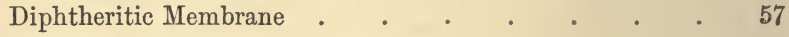

Hanging Drop Cultures . . . . . . $\quad$. 57

LEProsy-

Staining of Frozen Sections _ . . . . . . 57,58

\section{LESSON XIV}

Tuberculosis-

Staining of Fresh Tubercular Sputum . . . 59-61

Staining of Carbolised Sputum . . . . . . . 61,62

Staining of Frozen Sections of Tuberculous Tissues-

(a) Quick Method . . . . . . . 62,63

(b) Slow Method . . . . . . . .63,64

Staining of Paraffin Sections of Tuberoulous Tissues-

(a) Slow Method . . . . . . . . .64,65

(b) Quick Method . . . . . . . . 65

Staining of Paraffin Sections of Diphtheritic Membrane-

(a) Eosine Methylene-Blue . . . . . 65

(b) Weigert's Fibrin Method . . . . . 66

\section{LESSON XV}

Tuberculosis (concluded) -

(a) Mammalian Tubercle (Pure Cultures) . . . . 67

(b) Avian Tubercle (Pure Cultures) . . . . . . . 67 
Glanders-Horse's Luxg (Paraffin Sections)-

(a) Slow Method

(b) Quick Method . . . . . . . . . . . . . . . . . 68

Tetands Bacilli .

Actinomycosis (PARAfFin Sections)-

(a) Weigert's Fibrin Method . . . . . . 69

(b) Differential Staining of Clubs . . . . . 70

Pyamic Spleen (Paraffin Sections)-

Gram's Method .

Phagocytosis-

(a) Hanging Drop . . . . . . . . . . . . . 71,72

(b) Stained Specimens . . . . . . . . . 72, 73

(c) Local Immunity and General Infection . . . 73, 74

(d) Effect of Heat on Phagocytosis . . . . . 74

\section{LESSON XVI}

Phagocytosis (concluded)-

(a) Heated Frog . . . . . . . . . 75

(b) Anæsthetised Frog . . . . . . . 76

(c) Phagocytosis in a Hanging Drop . . . . . 76, 77

Chemiotaxis . . . . . . . . 77 
PART II

\section{BACTERIOLOGICAL ANALYSIS}

\section{LESSONS I AND II}

Cleaning and Sterilisation of Glass-

PAGE

(1) Cleaning of New Test-Tubes . . . . . 81

(2) Cleaning of Used Test-Tubes . . . . . 82

(3) Cleaning of Flasks and Beakers : . . . . 82,83

(4) Sterilisation . . . . . . . . 83

Preparation of Nutrient Media-

(1) Beef Broth . . . . . . . . . 83-85

(2) Glycerine Broth . . . . . . . . 85

(3) Grape-Sugar Broth . . . . . . . 85

(4) Meat Infusion . . . . . . . . . . 86

(5) Gelatine . . . . . . . . . . . . . . . . . . 86

(6) Grape-Sugar Gelatine . . . . . . . . 87

(7) 25 Per Cent Gelatine . . . . . . . . . . 87,88

(8) Carbolic Acid Gelatine . . . . . . . 88

(9) Peptone Solution . . . . . . . . . . 88,89

(10) Potato Tubes . . . . . . . . 89

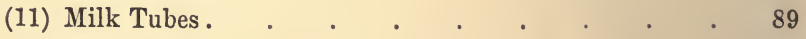

(12) Agar-Agar . . . . . . . . . . . . . 90, 91

(13) Glycerine Agar-Agar . . . . . . . . 91

(14) Grape-Sugar Agar-Agar . . . . . . . . 91, 92

(15) Blood Serum Tubes . . . . . . . . . 92 


\section{LESSON III}

EXAMINATION OF WATER-

PAGE

I. Quantitative Examination.
A. Tap Water-
(a) Plate Culture Method .
(b) Roll Tubes.
$.95,96$
B. Distilled Water .
C. Tank Water . . . . . . . 96
D. Effect of Sunlight on Water . . . . . $\quad$. 97

\section{LESSON IV}

Examination of Water (continued)-

II. Qualitative Examination.

A. Bacillus of Enteric Fever and Bacterium coli commune 98-100

\section{LESSON V}

Examination of Water (concluded)-

II. Qualitative Examination (concluded).

B. Vibrio choleræ Asiaticæ-

I. Peptone Method . . . . . . 101-103

II. Gruber's Method . . . . . . 103

III. Agar-Agar Plates . . . . . . . 103, 104

IV. Gelatine Plates . . . . . . 104

\section{LESSON VI}

Examination of MrLK-

I. Quantitative Examination . . . . . . 105

II. Qualitative Examination-

(1) Bacillus of Enteric Fever . . . . . . 106 
(2) Bacterium coli commune

(2) Bacterium coli commune . . . . . . 106

(3) Streptococcus pyogenes . . . . . . . . 106

(4) Bacillus diphtheriæ . . . . . . . . 106

(5) Bacillus tuberculosis (Van Ketel's Method) . . 107, 108

\section{LESSON VII}

Examination of Air and Dust-

I. Plate Culture Method . $\quad$. $\quad$. . . 109

II. Aspiration through Broth . . . . . 110

III. Filtration through Sugar . . . . . . 110,111

\section{LESSON VIII}

Examination of Air and Dust (concluded)-

IV. Anaërobic Germs in Air and Dust .

Examination of Soll-

A. Surface Soil-

(a) Aërobic Germs

(b) Anaërobic Germs

B. Tetanus and Malignaut Edema Bacilli-

(a) Growth in an Exhausted Flask . . . . 116

(b) Growth in Hydrogen . . . . . . . 116-118

(c) Fractional Separation of Tetanus Bacilli . . . . 118, 119

\section{LESSON IX}

Examination of Decomposing Meat-

A. Putrefaction-
(a) Aërobic Putrefaction
(b) Anaërobic Putrefaction . . . . . . . 120,121
(c) Sterilisation and Putrefaction . . . . . 121-122 
B. How to Examine a Sample of Unsound Meat . . 122

C. Trichina spiralis . . . . . . . . 123

D. Cysticercus . . . . . . . . . 123

E. Psorosperms . . . . . . . . . 124

Examination of Ice Creams-

(a) Quantitative Examination

(b) Qualitative Examination-

Bacillus of Enteric Fever . . . . . 125

Bacterium coli commune . . . . . 125

\section{LESSON $\mathrm{X}$}

Examination of Antiseptics and Disinfectants--

A. Method of Testing Antiseptics . . . . . 126

B. Methods of Testing Disinfectants-

(1) Koch's Method-

(a) Carbolic Acid . . . . . . . . 128

(b) Mercuric Chloride . . . . . . . 128

(2) Sternberg's Method . . . . . . . . 129

C. Disinfectant Action of Gases-
(a) Sulphur Dioxide
129,130
(b) Chlorine . . . . . . . . . . 131
(c) Ammonia . . . . . . . . . . 131

\section{LESSON XI}

Examination of an Animal dead of a Bacterial Disease-

(a) Anthrax . . . . . . . . . 132

(b) Pyocyaneus septicæmia . . . . . . . 132

(c) Cholera Asiatica . . . . . . . . . 132

Methods of Hardening and Embedding-

(a) Quick Method. . . . . . . . 133, 134

(b) Slow Method . . . . . . . . . . 134, 135 


\section{LESSON XIL}

Testing of Filters-

PAGE
A. Filter-paper .
136
B. Berkefeld Filter . . . . . . . . 136, 137
C. Effect of Use on a Berkefeld Filter . . . . . 137, 138

\section{PART III}

\section{BACTERIOLOGICAL CHEMISTRY}

\section{LESSON I}

Preparation of Metabolic Products of Micro-organisms-

(a) Sterilisation by Heat . . . . . . 141

(b) Sterilisation by Filtration . . . . . . 141

(c) Sterilisation by Heat and Filtration . . . . . 141, 142

(d) "Intracellular" Poisons . . . . . . 142

Test for Nitrous Acid in Cultures . . . . 143

\section{LESSON II}

Proteïnes-

(a) Bacillus pyocyaneus. . . . . . . . 144, 145

(b) Bacillus prodigiosus. . . . . . . 145

(c) Precipitation by Alcohol . . . . . . . 145, 146

Bacterial Extracts . . . . . . . . . 146 


\section{LESSON III}

Bacterial Pigments-

PAGE

(a) Bacillus pyocyaneus . . . . . . . 147, 148

(b) Bacillus prodigiosus . . . . . . . . . 149

\section{LESSON IV}

Peptones

Albumoses-

Proto-albumose . . . . . . . . 151

Deutero-albumose . . . . . . . . 151

\section{LESSON V}

Albumoses (concluded)-

Separation of Albumoses

$.152-154$

\section{LESSON VI}

Diphtheria Albumoses-

(a) From Cultures. . . . . . . . 155-157

(b) From Diphtheria Spleen . . . . . . 157

\section{LESSON VII}

Diphtheria Toxine-

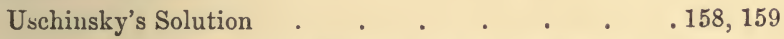

Action of Magnesium or Ammonium Sulphate on SulPHATE OF QUiNiNe . . . . . . . 160 


\section{LESSON VIII}

Ferments and Enzymes-

(a) Ferments and Enzymes in Yeast

(b) Action of Chloroform on Ferments and Euzymes . 162, 163

(c) Action of Moist Heat on Enzymes . . . . . 163, 164

(d) Separation of Enzymes . . . . . . . 164 (1) Barth's Method . . . . . . 164,165

\section{LESSON IX}

Ferments and Enzymes (concluded)-

(d) Separation of Enzymes (concluded)-

(2) Von Brücke's Method . . . . . . 167, 168

(e) Proteolytic Ferments of Micro-organisms . . . 168

(1) Bacillus prodigiosus . . . . . . . . 168

(2) Bacillus pyocyaneus . . . . . . . 168

(3) Vibrio Finkler-Prior . . . . . . 168

(4) Vibrio choleræ Asiaticæ . . . . . 168

(f) Tryptic Enzymes of Bacillus prodigiosus or Bacillus pyocyaneus . . . . . . . . 169-171

\section{LESSON X}

PTOMAÏNES-

(a) Cadaverine

(b) Putrescine

. $172-174$

. 172-174 


\section{PART I \\ GENERAL BACTERIOLOGY \\ LESSONS I-XVI}

5

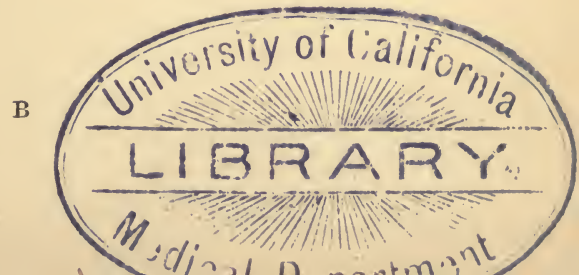



$38^{\circ} \mathrm{C}$. will show a copious white growth, while the other tube will show a pink or red growth. Now keep both tubes at $20^{\circ} \mathrm{C}$. The growth originally kept at $38^{\circ} \mathrm{C}$. may gradually develop pigment, but at times it has permanently lost its chromogenic properties.

(2) Inoculate two sloped agar-agar tubes with a single loop of a broth culture of Bacillus pyocyaneus (eighteen hours old), distributing the material over the entire surface of the agar-agar.

Expose one tube to the light and keep the other at the ordinary temperature of the room protected from the light.

Examine them on successive days : the tube exposed to the light shows restricted growth, or perhaps no growth at all, in any case limited production of pigment, while the other tube will show a copious growth, the culture medium at the same time becoming bright green. Light, therefore, has an inhibitory action on the growth and activity of the Bacillus pyocyaneus, which is more marked the longer the rays of the sun are allowed to act, and the less material is used for inoculation.

(3) Repeat the experiment with the Bacillus prodigiosus. Inoculate two sloped agar-agar tubes with a single loop of a pigmented broth culture of Bacillus prodigiosus (about a week old), distributing the material over the entire surface of the agar-agar.

Keep one tube opposite a sunny window at the ordinary temperature of the room, and the other at the same temperature but protected from the light. 
Growth will take place in both tubes, but the tube exposed to light will show less pigment than the tube kept in the dark. ${ }^{1}$

(4) The Bacillus prodigiosus requires oxygen for its pigment production.

(a) Draw out a fine capillary pipette and fill its bulb with a small quantity of a fresh broth culture of the Bacillus prodigiosus (eighteen hours old). This is best done in the following manner.

(b) Fuse both ends of the freshly made pipette, which is naturally sterile, and thrust it through the loosened cottonwool plug into the culture fluid, breaking its point by pushing it against the bottom of the tube.

(c) As the bulb of the pipette cools the liquid is gradually sucked up. When the pipette is full, withdraw it and again fuse the broken end.

(d) Now sterilise the other end by passing it several times quickly through the flame and push it through the loosened cotton-wool plug into a stab gelatine tube.

(e) When it has cooled down sufficiently, thrust it through the gelatine against the bottom of the tube in order to break the end of the pipette.

( $f$ ) Then withdraw it into the centre of the gelatine and apply gentle warmth to the bulb, until a minute drop of the liquid exudes.

(g) Now allow the bulb to cool again, and when the liquid has run back, withdraw the pipette. In this manner,

1 This is contrary to the experience of some observers, who state that the intensity of pigmentation varies directly with the amount of light supplied. 
provided the pipette was fine enough, the deeper part of the gelatine only is inoculated.

Keep the tube at the ordinary temperature of the room exposed to diffused light.

Gradually a whitish growth appears in the depth of the gelatine, which slowly extends to the surface and eventually breaks through. It will now quickly develop its typical red pigment.

(5) Inoculate a potato tube with Aspergillus niger by rubbing the material thoroughly over the slanting surface. Keep the tube in the cool incubator at $20^{\circ} \mathrm{C}$.

Examine after forty-eight hours: a white mould will be found on the surface of the potato. Later on a few black spots will appear on the white surface, and after a few weeks the whole growth will be black.

(6) Make an agar-agar streak culture of the Bacillus fluorescens and keep the tube at $20^{\circ} \mathrm{C}$.

Examine next morning: a copious growth has developed on the surface of the agar-agar and the culture medium has assumed a light green tint.

(7) Make a gelatine streak culture of Torula alba and keep the tube at $20^{\circ} \mathrm{C}$.

Examine after forty-eight hours : there is no liquefaction of the culture medium.

(8) Make a gelatine stab culture of Sarcina lutea and keep it at $20^{\circ} \mathrm{C}$.

Examine after forty-eight hours: there is a slight yellow growth with commencing liquefaction of the gelatine. 
Examine two weeks later: the gelatine is completely liquefied but clear, the yellow culture having sunk to the bottom of the tube.

(9) Make a gelatine stab culture-

(a) with Staphylococcus pyogenes aureus;

(b) with Staphylococcus cereus flavus, and keep the two tubes at $20^{\circ} \mathrm{C}$.

Examine after forty-eight hours: Staphylococcus pyogenes aureus liquefies gelatine, rendering it at the same time turbid.

Staphylococcus cereus flavus does not liquefy it. 


\section{LESSON II}

Types of Micro-organisms-Microscopic Examination of Bacteria, stained and unstained-Staining of Micro-organisms with Simple Basic Aniline Dyes-Preparation of Cover-glass Films.

\section{Types of Micro-organisms}

Examine microscopically the broth cultures supplied.

Cultures supplied-

1. Streptococcus pyogenes.

2. Staphylococcus pyogenes aureus.

3. Bacillus filamentosus.

4. Torula alba.

5. Sarcina lutea.

(a) Clean a cover-glass (No. 1, $\frac{5}{8}$ in. by $\frac{5}{8}$ in.) with alcohol, and pass it through the flame in order to sterilise it.

(b) Under aseptic precautions with a platinum loop remove a drop of the culture and place it on the centre of the cover-glass.

(c) Gently press the cover-glass on a clean slide and examine the specimen with a $\frac{1}{12}$ in. oil immersion, using a narrow diaphragm and the concave mirror.

Make sketches of the various specimens. 


\section{Staining of Micro-organisms with Simple Basic}

\section{Aniline Dyes}

Examine the same organisms after staining with ordinary aniline dyes.

Prepare staining solutions-

(1) To a watch-glass containing distilled water add two to three drops of a filtered concentrated alcoholic solution of fuchsine.

(2) To a watch-glass containing distilled water add two to three drops of a filtered concentrated alcoholic solution of gentian-violet.

(3) Into a watch-glass filter a little concentrated aqueous solution of methylene-blue.

(a) Clean cover-glasses as before and transfer a drop of the broth culture to the centre of the cover-glass, and with the platinum needle spread it uniformly over the surface.

(b) Allow the film to dry in the air, covering it up with a watch-glass so as to protect it from the dust.

(c) When dry, pass the cover-glass three times through the flame, holding it in a pair of forceps with the smeared. surface upwards.

(d) Now place it in a solution of 20 per cent acetic acid for five to ten minutes, by which means the ground substance is removed and cleared up.

(e) Wash the acetic acid off with distilled water, and dry the cover-glass between folds of filter-paper. ${ }^{1}$

${ }^{1}$ It is not always necessary to clear the ground substance with acetic acid. 
( $f$ ) Once more dry the film in the air and pass it through the flame.

(g) Now stain the specimen by floating it with the film surface downwards on the staining solution.

For Streptococcus pyogenes and Torula alba use fuchsine.

For Bacillus filamentosus use methylene-blue.

For the Staphylococcus pyogenes aureus and Sarcina lutea use gentian-violet.

Leave the films in the stain for some time (two to five minutes), then wash in water and dry between folds of filter-paper; clean the unsmeared surface, mount in water, and examine under a high power and Oc. 4, using no diaphragm, and the plane reflector. ${ }^{1}$

If the specimen is successful, float it off the slide, dry it again, and mount it permanently in xylol balsam. Examine it with a $\frac{1}{12}$ in. oil immersion.

Make drawings.

If the specimen is not sufficiently stained, place it once more in the staining fluid, and proceed as above.

If the specimen is overstained, it is better to prepare a fresh one. Weak acetic acid $\left(\frac{1}{1000}\right)$, however, may be used to decolourise it.

Micro-organisms vary greatly in regard to their affinity for dyes, and, again, some dyes stain more quickly than others. Thus aqueous methylene-blue hardly ever over-

${ }^{1}$ Stained specimens should always be examined without a diaphragm, with an Abbé condenser and a plane reflector; unstained specimens with a narrow diaphragm and a concave reflector without an Abbé condenser. 
stains, while fuchsine frequently does so. It is impossible to give definite rules as to how long a film should be left in the stain. It is safest to examine the specimen off and on in water, and if it be understained, to continue the process of staining.

Sarcinae should be stained with very dilute solutions, since they easily overstain. 


\section{LESSON III}

Staining of Bacteria (continued)-Löffler's Methylene-blue-Examination of Moulds-Hanging Drop Cultures.

\section{Löffler's Methylene-blue}

Prepare and stain cover-glass films of the various cultures made during the previous lesson.

(1) Bacillus prodigiosus : agar-agar culture.

(a) On a clean cover-glass place a small drop of sterile distilled water. With a sterilised platinum needle remove a trace of the culture, and, mixing it with the drop of water, spread it uniformly over the cover-glass.

(b) Allow the film to dry in the air, and then pass the cover-glass three times through the flame and proceed as before (vide p. 9), staining the film with methylene-blue.

If necessary, clear the film with acetic acid (20 per cent).

Instead of the ordinary aqueous solution of methyleneblue Löffler's methylene-blue may be used with advantage. This is one of the best and most certain of staining solutions, and gives almost always good results. It is prepared in the following manner :- 
Concentrated alcoholic solution of methylene-blue, 3 vols.

Caustic potash solution $1: 10,000,10$ vols.

A convenient method of staining is to keep the staining solution in a wide-necked glass-stoppered pot, and to hold the film in the staining solution with a pair of forceps, gently moving it about for one or two minutes. Then transfer it to water and wash off the superfluous stain.

(2) Staphylococcus pyogenes aureus : liquefying gelatine culture.

(a) With a loop remove a little of the liquefied gelatine and spread it carefully over a clean cover-glass.

(b) Allow the film to dry in the air, and then pass it three times through the flame.

(c) Remove the gelatine by means of acetic acid in the usual manner, or by floating the film on warm water for five to ten minutes.

(d) Stain with Löffler's methylene-blue, wash in water, dry with blotting-paper, and mount in Canada balsam.

Examine with $\frac{1}{12}$ in. oil immersion.

(3) Torula alba : non-liquefying gelatine streak culture.

Proceed in exactly the same manner as in the case of Bacillus prodigiosus (vide p. 12).

Stain with aqueous gentian-violet or aqueous fuchsine solution or with Löfller's methylene-blue.

Examine with $\frac{1}{12}$ in. oil immersion.

\section{Examination of Moulds}

Aspergillus niger : potato culture. 
(a) With a platinum loop remove a little of the culture and place it on a clean slide.

(b) Add a drop or two of caustic potash (1-5 per cent solution $)^{1}$ and allow this to act for five to ten minutes.

(c) With filter-paper soak up as much of the caustic potash as possible.

(d) Mount in Farrant's solution, and examine with a low and a high power, using a narrow diaphragm.

Make a drawing of the fungus.

\section{Hanging Drop Cultures}

Make hanging drop cultures of

(1) Streptococcus pyogenes.

(2) Bacillus filamentosus.

(a) Take four clean hollow-ground slides. With a brush paint a ring of vaseline around the periphery of the hollow chambers.

(b) Sterilise four clean cover-glasses by passing them three times slowly through the flame.

(c) Place the sterilised cover-glasses on a sterilised piece of wire gauze on a tripod and cover it over with a glass globe sterilised by washing it thoroughly with corrosive sublimate $1: 1000$. The wire gauze is easily sterilised by heating it to redness over a Bunsen flame.

(d) With a sterilised platinum loop place a drop of sterile broth on the centre of each cover-glass, after allowing the cover-glass to cool.

1 Instead of caustic potash a solution of 50 per cent alcohol, to which a few drops of ammonia have been added, may be used. 
(e) With a straight sterilised platinum needle inoculate two of the drops with a minimal trace of the Streptococcus broth culture, and two with a minimal trace of Bacillus filamentosus.

$(f)$ Now carefully place each cover-glass on the vaseline ring around the hollow on the slide, the drop facing, of course, downwards.

(g) Gently press the cover-glass down on the vaseline, so as to completely shut off all air from the chamber.

(h) Label the slides and place them in a moist chamber and keep the latter at $38^{\circ} \mathrm{C}$.

Examine the hanging drops after twenty-four hours with a high power, using a narrow diaphragm. In the one case there will be a pure culture of streptococci, in the other a pure culture of filamentous bacilli.

\section{Examination of Bacteria in a Hanging Drop}

To study the motility of micro-organisms, hanging drops should be made.

(a) Take an ordinary clean slide.

(b) With a cork-borer cut a small ring $\frac{3}{8}$ to $\frac{4}{8}$ in. in diameter out of filter-paper, four to eight layers thick.

(c) Trim the perforated filter-paper so as to fit the slide, moisten it with water, and place it on the centre of the slide.

(d) With a sterilised platinum loop place a drop of a fresh broth culture of the Bacillus filamentosus on the centre of a clean sterilised cover-glass. 
(e) Place the cover-glass drop downwards over the central perforation of the moistened filter-paper.

Examine the drop with a high power, using a narrow diaphragm.

Focus for the margin of the drop, and then move the specimen till a bacillus appears in the field.

Now place a drop of oil on the cover-glass, and examine with a $\frac{1}{12}$ in. oil immersion.

Distinguish between true and Brownian movements. 


\section{LESSON IV}

Staining of Hanging Drop Cultures-Gram's Method of StainingStaining of Pus-Curdling Ferment.

\section{Staining of Hanging Drop Cultures}

Bacillus filamentosus.

(a) Carefully remove the cover-glass and allow the drop culture to dry in the air.

(b) Wipe off as much of the vaseline as possible.

(c) Pass the film three times through the flame.

(d) Carefully stain in Löfler's methylene-blue for two to three minutes.

(e) Wash very gently in water.

(f) Dry the film between folds of filter-paper, and when it is quite dry mount in xylol balsam.

Examine it with a high power, and then with $\frac{1}{12}$ in. oil immersion. Observe the beautiful network of filaments.

\section{Gram's Method of Staining}

Streptococcus pyogenes.

Stain the hanging drop cultures of Streptococcus pyogenes by this method. 
Solutions required :-

(a) Aniline Gentian-Violet is prepared in the following manner :-

(a) First make aniline water by shaking up $4 \mathrm{cc}$. of aniline oil with 100 cc. of distilled water for one to two minutes.

(b) Filter the resulting emulsion through filter-paper moistened with distilled water.

(c) To 100 cc. of aniline water add 11 cc. of a concentrated alcoholic solution of gentian-violet.

Shake the mixture thoroughly and always filter it before use.

This solution does not keep well, and should therefore be prepared in small quantities.

( $\beta$ ) Gram's Iodine Solution :-

Iodine crystals

Potassium iodide

Distilled water
1 gramme

2 grammes

$300 \mathrm{cc}$.

( $\gamma$ ) Absolute Alcohol.

Method of staining :-

(a) Prepare the hanging drop culture of the Streptococcus, as described before for Bacillus filamentosus, and when the film is dry and has been passed through the flame, place it first in alcohol for one to two minutes.

(b) Without drying it, transfer it to the aniline gentianviolet, and leave it in the stain for half a minute to one minute.

(c) Soak up the superfluous stain with blotting-paper.

(d) Now place it in Gram's solution of iodine for half a minute to one minute. 
(e) Soak up the superfluous iodine solution, and gently wash in absolute alcohol, until no more stain comes away.

$(f)$ Quickly wash the alcohol off in water, and dry the film between folds of blotting-paper.

(g) When the cover-glass is quite dry, mount it in xylol balsam.

Examine with a high power, and then with $\frac{1}{12}$ in. oil immersion: long chains of Streptococci will be seen, stained dark violet.

Prepare Cover-glass Films of a Liquefied Culture of Staphylococcus Pyogenes Aureus, and Stain by Gram's Method.

(a) Prepare the film and pass it through acetic acid in the usual manner (vide p. 9).

(b) Wash off the acetic acid with water, dry the film and pass it three times through the flame.

(c) Now place the film in absolute alcohol for one to two minutes, and stain with aniline gentian-violet, and continue as above with Gram's solution, alcohol, etc.

\section{Staining of Pus}

(1) With methylene-blue.

(2) By Gram's method.

(a) With a platinum loop smear a thin film of pus on two clean cover-glasses.

(b) Allow the films to dry in the air, and then pass them three times through the flame, keeping the smeared surface upwards. 
(c) Place the films in 20 per cent acetic acid for three to five minutes.

(d) Wash them in water, dry them between folds of blotting-paper, and pass them again through the flame. The films are now ready for staining.

(1) Löfler's methylene-blue.

(a) Place one film in the stain for two to five minutes.

(b) Wash in water, dry and mount in xylol balsam.

Examine with $\frac{1}{12}$ in. oil immersion: observe the leucocytes and cocci (Staphylococci or Streptococci, or both; many probably in the leucocytes).

(2) Gram's method.

(a) Place the other film in absolute alcohol for one to two minutes.

(b) Stain it in aniline gentian-violet for one to two minutes.

(c) Remove the superfluous stain with blotting-paper.

(d) Now place in Gram's solution of iodine for half a minute, until the specimen turns black.

(e) Soak up the superfluous iodine solution.

$(f)$ Wash in alcohol till the film is almost colourless.

(g) Pass quickly through a diluted alcoholic solution of eosine, which stains the leucocytes and ground substance pink.

(h) Wash in water, dry and mount in xylol balsam.

Examine with a high power, and then with $\frac{1}{12}$ in. oil immersion : the pus cocci are stained dark violet, and also the chromatine of the nuclei; the ground substance and protoplasm of the leucocytes are pink. 


\section{Prepare Cultures of Bacillus Anthracis}

From the agar-agar culture supplied inoculate-

(1) a potato tube;

(2) a broth tube;

(3) an agar-agar tube (streak);

(4) a gelatine tube (stab).

Place (1) (2) and (3) in the warm incubator, and (4) in the cool incubator.

Examine after forty-eight hours : observe the characteristic growths in the broth and gelatine. The latter is slowly liquefied.

\section{Curdling Ferment}

Some micro-organisms will coagulate milk, others will not.

Inoculate four milk tubes with-
(1) Bacillus fluorescens;
(2) Bacterium coli commune;
(3) Bacillus anthracis ;
(4) Bacillus prodigiosus.

Place the inoculated tubes in the warm incubator.

Examine the tubes after forty-eight hours: the milk in (1) and (2) will be coagulated, that in (3) and (4) will not. 


\section{LESSON V}

Bacillus Anthracis-Mode of Growth on Various Media-Asporogenous Cultures-Acid Formation by Virulent Anthrax Bacilli-Methods of Attenuation-Impressions (Sloped Gelatine)-Methods of Staining.

\section{Bacillus Anthracis}

(1) Examine the tubes inoculated with Bacillus anthracis and note the mode of growth on the various media.

(2) Prepare a streak culture of Bacillus anthracis on neutral litmus agar-agar, and place it in the warm incubator.

Examine it after forty-eight hours, and notice the change in the colour of the litmus. The colour gradually assumes a reddish tint on account of the acid produced by the bacillus.

(3) Prepare two hanging drop cultures of Bacillus anthracis (vide p. 14), and place them in the warm incubator.

(4) Inoculate a broth tube containing carbolic acid (1:1000), and keep it at $38^{\circ} \mathrm{C}$.

Examine it microscopically a week later : no spores will be found (asporogenous growth). 
(5) Inoculate a broth tube and place it in a water incubator at $42.5^{\circ} \mathrm{C}$.

Examine it microscopically a week later : no spores will be found, and the bacillus, moreover, is attenuated and less virulent.

(6) Inoculate two neutral litmus agar-agar tubes: one with ordinary virulent bacilli and the other with the attenuated bacillus.

The latter produces less acid.

(7) (a) Inoculate a tube containing a little sterilised $\cdot 75$ per cent saline solution with three platinum loops of an agar-agar culture of Bacillus anthracis.

(b) Shake the inoculated tube vigorously, and from it, with a platinum loop, inoculate a sloped gelatine tube, thoroughly streaking the surface of the culture medium.

(c) Place the tube in the cool incubator, and when small colonies appear they are to be worked up by means of impression specimens (vide infra p. 26).

(8) Staining of Bacillus anthracis.

(a) Prepare cover-glass specimens in the usual manner-

(1) of a broth culture of Bacillus anthracis;

(2) of a gelatine culture;

(3) of an agar-agar culture.

(b) Stain films-

(a) With Löffler's methylene-biue after having cleared with acetic acid.

$(\beta)$ With gentian-violet after having cleared with warm water. 
(y) According to Gram's method.

Examine with a high power and also with $\frac{1}{12}$ in. oil immersion: bacilli and spores will be found, the spores remaining unstained.

(9) Prepare a hanging drop of a broth culture of the Bacillus anthracis, and examine it with $\frac{1}{12}$ in. oil immersion. The Bacillus anthracis is not motile. 


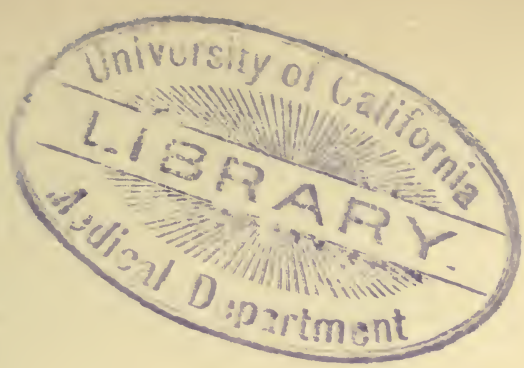

LESSON VI

Bacillus Anthracis (continued)-Hanging Drop Cultures-Methods of Staining Impressions-Examination of Fresh Tissues by means of Cover-glass Films-Staining of Leucocytes for Eosinophile Granules.

\section{Bacillus Anthracis (continued)}

(1) ExAmine the litmus agar-agar tube inoculated with Bacillus anthracis, and note the gradual disappearance of the blue or purple colour.

(2) Examine the hanging drop cultures with a high power.

\section{Make a drawing.}

Stain (a) one of them with Löffler's methylene-blue in the usual manner (vide p. 13); (b) the other according to Gram's method.

(3) Prepare a cover-glass specimen of the culture in carbolic acid broth and stain it with Löffler's methyleneblue, or according to Gram's method.

Note the absence of spores.

(4) Inoculate a litmus agar-agar tube from the broth culture kept at $42.5^{\circ} \mathrm{C}$., and another litmus agar-agar tube from an ordinary broth culture grown at $38^{\circ} \mathrm{C}$. 
Place the former in the water incubator at $42.5^{\circ} \mathrm{C}$., and keep the latter at $38^{\circ} \mathrm{C}$.

Compare them after forty-eight to eighty hours and notice the difference in the tint of the litmus. The latter or more virulent culture decolourises the agar-agar more rapidly.

\section{Impression Specimens}

(a) Examine the sloped gelatine tubes (vide Lesson V. 7, p. 23) with a magnifying-glass for colonies of anthrax bacilli.

(b) If found, rapidly dip the gelatine tube into boiling water, having previously removed the cotton-wool plug. This momentary heating will free the gelatine from the sides of the tube.

(c) Slide the gelatine block out on to a cool black glass plate with the culture surface upwards, and trim the sides of the gelatine block with a sharp scalpel dipped in methylated spirit.

(d) Select small superficial colonies and gently press a clean cover-slip down over them.

(e) Now carefully remove the cover-glass with the impression of the colonies adhering to its under surface.

(f) Gently warm the cover-glass and then pass it three times through the flame.

(g) Stain with Löffler's methylene-blue, or according to Gram's method, and mount.

Examine under a high power, and make a drawing of the impression. 
Examination of Fresh Tissues by means of Cover-Glass Films

Examine the spleen of the white mouse dead of anthrax, for the presence of Bacillus anthracis, by means of cover-glass specimens.

(a) Pin the animal out on a small board washed with sublimate solution $\left(\frac{1}{1000}\right)$.

(b) Moisten the hair on the abdomen and chest with methylated spirit.

(c) Carefully reflect the skin of the abdomen and chest.

(d) Heat a small glass rod to redness in the blow-pipe, and rub the surface of the abdomen with it, especially along the linea alba, and on each side along the costal arches.

(e) With a sterilised pair of forceps and scissors carefully open the abdominal cavity in the middle line, make two transverse cuts along the costal arches, and throw the flaps outwards.

$(f)$ With a fresh pair of sterilised forceps and scissors remove a small piece of the swollen spleen and gently smear it over a clean cover-glass. Prepare four films in this way.

(g) Allow them to dry in the air, and then pass them three times through the flame.

(h) Stain them according to the following methods :-

(1) In methylene-blue without previously passing the film through acetic acid.

(2) First pass the film through acetic acid in the usual manner and then stain with Löfler's methylene-blue. 
(3) Stain a film with eosine and methylene-blue.

(a) Dip the cover-glass for a few seconds in a widenecked pot containing 5 per cent solution of eosine in 50 per cent alcohol.

(b) Wash it in water, and dry it with blotting-paper.

(c) Pass it three or four times through the flame, and now stain quickly in Löffler's methylene-blue.

This method stains the eosinophile granules of the leucocytes and also brings out the red corpuscles.

(4) Stain a film according to Gram's method, first passing the specimen through acetic acid. Counterstain with eosine.

In each case mount in xylol balsam and examine with $\frac{1}{12}$ in. oil immersion.

Note the absence of spores and the relation of the bacilli to the leucocytes. 


\section{LESSON VII}

Bacillus Anthracis (continued)-Plate Cultures-Staining of SporesSeparation of Bacillus Subtilis from Hay Infusion.

\section{Bacillus Anthracis (continued)}

ExAMrNe the litmus agar-agar cultures of Bacillus anthracis made on the last occasion.

Note the difference in tint of the two tubes.

\section{Plate Culture Method}

(a.) Take a broth culture of Bacillus anthracis (fortyeight hours old) and three gelatine tubes.

(b) Liquefy the gelatine tubes in the warm incubator.

(c) Shake the broth culture, and from it inoculate the first gelatine tube with three platinum loops, and label it I.

(d) From I., after shaking, inoculate the second gelatine tube with three platinum loops, and label it II.

(e) From II. similarly inoculate the third tube, and label it III.

(f) Take three sterilised Petri's capsules and place them on moist filter-papers, and label them I. II. and III.

(g) Now heat the mouths of I. II. and III. in the flame and allow them to cool. 
(h) Pour out each gelatine tube, after gently shaking it, into the corresponding capsule.

(i) Allow the gelatine to set in a uniform layer, and when set, place the capsules in the cool incubator.

Examine on the next occasion: colonies will appear on each plate, naturally most numerously on plate I. and least on plate III. (vide infra p. 34).

\section{Staining of Spores}

Bacillus filamentosus, or anthracis, or megatherium (agaragar or potato cultures).

Solutions required :-

(a) Ehrlich's, Löffler's, or Ziehl's fuchsine.

( $\beta$ ) Acid alcohol : alcohol 97 cc., hydrochloric acid 3 cc.

( $\gamma$ ) Löffler's methylene-blue.

Ehrlich's fuchsine: Aniline water 100 cc.

Concentrated alcoholic solution of fuchsine $11 \mathrm{cc}$.

This staining solution does not keep well, and therefore only small quantities should be made at a time.

Löfler's fuchsine: Aniline water $100 \mathrm{cc}$.

Fuchsine crystals 4 to 5 grammes.

1 per cent solution of caustic soda 1 cc.

Shake till dissolved (about half an hour). 
Ziehl's fuchsine: 5 per cent aqueous solution of carbolic acid $100 \mathrm{cc}$.

Concentrated alcoholic solution of fuchsine $11 \mathrm{cc}$.

All these solutions must be filtered before use.

First Method.

(a) Prepare a film in the usual manner and pass it three times through the flame.

(b) Filter some of the fuchsine solution into a small evaporating dish and gently warm it, keeping it just steaming.

(c) Float the cover-glass, film surface downwards, on the warm stain and continue gentle warming for twenty to thirty minutes.

(d) Now wash the specimen in water.

(e) Dip it for a few seconds in the acid alcohol, and at once wash it again in water to remove the acid.

(f) Examine it in water, under a high power, for spores, which should appear as round or oval red bodies, the bacilli themselves being faintly pink or unstained.

If the spores are unstained or too much decolourised, place the film back in the fuchsine solution, and decolourise for a shorter time in the acid alcohol.

If, on the other hand, the bacilli are not sufficiently decolourised, dip the cover-glass once more into the acid alcohol.

(g) When the spores are well stained, and the bacilli practically unstained, dry the specimen with blotting-paper and pass it three times through the flame. 
(h) Now counterstain with Löffler's methylene-blue, wash in water, dry and mount.

Examine with $\frac{1}{12}$ in. oil immersion : the bacilli are blue and the spores red.

Make a drawing.

The spores of the Bacillus filamentosus and the Bacillus megatherium are more easily stained than those of the Bacillus anthracis.

Second Method.

(a) Prepare a film in the usual manner.

(b) Pass it three times through the flame.

(c) Place it for two minutes in absolute alcohol, and for further two minutes in chloroform.

(d) Wash the film in water, dry it between blottingpaper, and pass it again three times through the flame.

(e) Leave it for two minutes in a 5 per cent solution of chromic acid, and then wash it in water, and dry it between blotting-paper.

(f) Once more pass it three times through the flame, and place it in a warm solution of carbol fuchsine for five to fifteen minutes.

(g) Rinse the film in water, and decolourise in 5 per cent sulphuric acid, till the-specimen is faintly pink.

(h) Wash it in water and counterstain in malachite green.

(i) Wash again, dry and mount.

The spores are red and well stained, the bacilli green. 


\section{Separation of the Hay Bacillus (Bacillus subtilis) out of Hay Infusion.}

Six tubes containing hay infusion are supplied.

Heat them for five, ten, fifteen, twenty, twenty-five and thirty minutes respectively in water at $80^{\circ} \mathrm{C}$.

From each tube inoculate a broth tube with three platinum loops of the infusion and label them.

Keep the six tubes in the cool incubator and examine them on the next occasion.

Pure cultures of the hay bacillus will probably be obtained. 


\section{LESSON VIII}

Bacillus Anthracis (concluded)-Impression Specimens (Gelatine Plates)

-Staining of Anthrax Bacilli in Tissues-Staining of Flagella.

\section{Bacillus Anthracis (concluded)}

WiтH a dissecting microscope examine the plates, made on the previous occasion, for colonies of anthrax bacilli.

Observe the characteristic appearance of the colonies.

Now place a clean cover-glass over a colony and examine it with a high power.

Make a drawing.

Impression specimens.

(a) Take a perfectly clean cover-glass, sterilise it by passing it through the flame, and place it over a suitable colony.

(b) With a needle carefully press the cover-glass down, until the colony is slightly flattened out.

(c) With a sterilised needle lift the cover-glass with the colony adhering to it from the surface of the gelatine; then proceed as described before (vide p. 26). 


\section{Staining of Tissues for Anthrax Bacilli (Frozen Sections)}

(1) Simple staining with Löfler's methylene-blue.

Tissues supplied: lung, kidney, liver and spleen of a mouse dead of anthrax.

(a) Place a section in Löfller's methylene-blue for five minutes.

(b) Wash it in distilled water for half a minute.

(c) Then decolourise it for a quarter to half a minute with very dilute acetic acid (one to two drops of glacial acetic acid to a watch-glassful of water), till the section is pale blue.

(d) Again wash it in water for half a minute.

(e) Now place it in absolute alcohol for half a minute to one minute.

(f) Clear the section in xylol. ${ }^{1}$

(g) Transfer the specimen to a slide with a piece of cigarette-paper.

(h) Mount it in xylol balsam.

Examine it with low and high powers and $\frac{1}{12}$ in. oil immersion.

(2) Double staining with methylene-blue and eosine.

Prepare the following solution (Czinzinski's solution) :-

1 Instead of xylol other clearing media may be used, as bergamot oil, a mixture of turpentine and creasote, or cedar-wood oil. 
Concentrated aqueous solution of methylene-blue Eosine .

Absolute alcohol Distilled water

$50 \mathrm{cc}$.

5 gramme

$70 \mathrm{cc}$.

$130 \mathrm{cc}$.

(a) Keep the section in absolute alcohol for five minutes.

(b) Transfer it to the stain for four to twelve hours.

(c) Wash it in water till the section appears pink, and shows hardly any trace of blue.

(d) Dehydrate, clear, and mount in the usual manner.

Examine with low and high powers. The bacilli are stained blue and the tissues pink.

(3) Double staining with picrocarmine and Löfler's methyleneblue.

(a) Place the section in alcohol for half a minute.

(b) Then wash it in water and place it in a solution of picrocarmine and water (equal parts) for ten to twenty minutes.

(c) Wash it in spirit, to which a few drops of hydrochloric acid have been added, for half a minute to one minute.

(d) Wash it in water.

(e) The sections are now stained red, and should be placed in methylene-blue and treated as described before.

(4) Staining of sections by Gram's method.

(a) Place the section in alcohol for half a minute. 
(b) From the alcohol transfer it directly to filtered aniline gentian-violet and allow it to stain for two to ten minutes.

(c) Rinse the section in distilled water.

(d) Now place it in Gram's iodine solution till it is black (about two minutes).

(e) Transfer it to absolute alcohol for a quarter to half a minute, and thence to

(f) Acid alcohol (alcohol with 3 per cent hydrochloric acid) for not more than ten seconds.

(g) Again wash it in alcohol until the section appears colourless, or if previously stained with picrocarmine, until the section again becomes red.

(h) Clear it in xylol or turpentine and creasote.

(i) Transfer it to a slide with a cigarette-paper.

(k) Mount it in Canada balsam.

Examine with low and high powers and $\frac{1}{12}$ in. oil immersion: the bacilli appear dark blue on a yellowish or red background.

\section{Cholera}

Inoculate-

(1) a gelatine tube (stab), an agar-agar tube (streak), and a broth tube with Vibrio choleræ Asiaticæ;

(2) a gelatine tube (stab), an agar-agar tube (streak), and a broth tube with Vibrio Finkler-Prior;

(3) a gelatine tube (stab), an agar-agar tube (streak), and a broth tube with Vibrio Metchnicovi. 
Place the gelatine tubes in the cool incubator at $22^{\circ} \mathrm{C}$., the others in the warm incubator at $38.5^{\circ} \mathrm{C}$.

\section{Staining of Flagella (Van Frmengem)}

Prepare the following solutions :-

(a) Osmic Acid (2 per cent solution) 1 part.

Tannin (10 to 25 per cent solution) 2 parts.

To each $100 \mathrm{cc}$. of the tannin solution add four or five drops of glacial acetic acid.

( $\beta)$ Nitrate of silver ( $\cdot 25$ to $\cdot 5$ per cent solution).

$(\gamma)$ Gallic Acid . . . 5 grammes. Tannin . . . 3 grammes.

Fused acetate of soda . 10 grammes.

Distilled water . . $350 \mathrm{cc}$.

Boil the cover-slips to be used in the following solution:-

Potassium bichromate

Concentrated Sulphuric Acid Water
60 grammes.

60 grammes. $1000 \mathrm{cc}$.

Then wash them repeatedly in water. Keep thom in absolute alcohol and before use allow them to dry, without wiping, by placing them in a vertical position, protected from dust.

Bacillus of typhoid fever and Vibrio cholerce Asiatico.

Carefully suspend one or two loops of an agar-agar culture (ten to eighteen hours old) in a watch-glassful of distilled water. 
(a) With a single loopful of this "suspension" prepare a cover-glass film and allow it to dry in the air.

(b) Fix it by passing it three times through the flame, holding the specimen in the fingers, so as to avoid overheating.

(c) Pour a few drops of solution $(a)$ on the film and allow them to act for half an hour. ${ }^{1}$

(d) Wash very carefully in a large excess of distilled water, and then in alcohol.

(e) Now keep it for three to five seconds in solution $(\beta)$.

$(f)$ Without washing, pass quickly through solution $(\gamma)$.

(g) Wash again in a fresh quantity of solution $(\beta)$, moving the specimen about gently and withdrawing it when the solution begins to turn.black.

(h) Wash it thoroughly in several changes of distilled water.

(i) Dry it carefully between blotting-paper.

Mount it first in water and examine it with $\frac{1}{12}$ in. oil immersion, and if the specimen be satisfactory, mount it permanently in xylol balsam.

If the flagella are not sufficiently stained, float the film off the slide and begin again at $(f)$.

Care must be taken to change the nitrate of silver solution as soon as any precipitation shows itself. This is an easy and very trustworthy method.

1 At a temperature of $60^{\circ} \mathrm{C}$. five minutes is sufficient. 


\section{LESSON IX}

Examination of Vibriones Choleræ Asiaticæ, Finkler-Prior, and Metchnicovi in Cultures and in the Animal Body-Plate Cultures - Germicidal Action of Sunlight-Hanging Drops.

\section{Cholera (continued)}

(1) Examine and compare the tubes inoculated with Vibrio choleræ Asiaticæ, Vibrio Finkler-Prior, and Vibrio Metchnicovi respectively.

(2) Prepare cover-glass specimens of Vibrio choleræ Asiaticæ, Vibrio Finkler-Prior, and Vibrio Metchnicovi in the usual manner.

Stain them with dilute aniline gentian-violet or dilute carbol fuchsine, as follows :-

(a) Filter some of the staining solution into a watchglass and dilute it with alcohol (equal parts).

(b) Stain for about three minutes, previously clearing the specimen with acetic acid, if necessary.

(c) Wash in water, dry and mount.

Examine with $\frac{1}{12}$ in. oil immersion and compare the three forms. 
(3) Prepare cover-glass specimens of Spirillum rubrum (gelatine stab culture).

(a) Melt the gelatine tube in the warm incubator.

(b) Prepare films and pass them three times through the flame in the usual manner.

(c) Wash the films in warm water for two to five minutes, in order to remove the gelatine.

(d) Stain in dilute aniline gentian-violet or carbol fuchsine, as above.

(e) Wash in water, dry and mount.

Examine with $\frac{1}{12}$ in. oil immersion.

(4) From a liquefied gelatine culture of Spirillum rubrum prepare a gelatine stab culture and keep it at $22^{\circ} \mathrm{C}$.

(5) Prepare hanging drop cultures of Vibrio choleræ Asiaticæ, using 1 per cent peptone solution.

Place them in the warm incubator.

(6) Examine the peritoneal fluid of the guinea-pig supplied for Vibrio cholerce Asiaticce.

(a) Open the abdominal cavity of the animal in the usual manner (vide p. 27).

(b) Make a capillary pipette, and draw up some of the peritoneal fluid into its bulb. .

(c) Prepare films from the fluid removed, in the usual manner, and pass them three times through the flame.

(d) Clear them with acetic acid and then stain with aniline gentian-violet, as already described (vide supra).

Examine with $\frac{1}{12}$ in. oil immersion. 
(e) Other films should be stained with eosine and methylene-blue, without previously clearing them with acetic acid (vide p. 28).

Examine with $\frac{1}{12}$ in. oil immersion : note the eosinophile granules in the leucocytes.

\section{Plate Cultures of Vibrio Choleræ Asiaticæ}

Open the thorax (of a guinea-pig inoculated intraperitoneally with the Vibrio cholerae Asiaticae) in the following manner :-

(a) Moisten the skin with spirit.

(b) Reflect it freely on both sides.

(c) Cauterise the exposed surface, and with a pair of sterilised scissors and forceps remove the sternum.

(d) Now cauterise with a heated glass rod a spot on the surface of the heart.

(e) Thrust a sterile capillary pipette, the ends of which have been broken off, through the heart's wall into an auricle or ventricle and remove a trace of blood by suction, and fuse the end to which suction has been applied.

$(f)$ Loosen the cotton-wool plug of a liquefied gelatine tube and insert the pipette into the test-tube by carefully pushing it through the cotton-wool.

(g) Push the pipette into the lumen of the tube, till it almost touches the gelatine.

(h) Apply very gentle heat to the bulb, till a drop of blood falls into the gelatine.

(i) Withdraw the pipette and shake the inoculated tube. 
(k) From this tube inoculate two other tubes in the ordinary manner, and pour three plates as described in a former lesson (vide p. 29).

Keep the plates at $22^{\circ} \mathrm{C}$. and examine them from day to day.

\section{Action of Sunlight on the Vibrio Choleræ Asiaticæ}

To demonstrate the action of sunlight on cholera vibrios take two agar-agar plates (Petri's capsules) ${ }^{1}$ which have been kept in the warm incubator for twelve to twenty-four hours.

With a sterilised brush gently paint the surface of the agar-agar with a dilute young broth culture of cholera vibrios.

Over the lid of each capsule gum a piece of black paper with a large figure or letter cut in it, and expose the plates to the sun for four to six hours.

Then transfer the plates to the warm incubator.

Examine them on the next day: there will be no growth (or only a limited growth) on the agar-agar over those areas which were unprotected from the action of the light by the black paper.

1 For preparation of agar-agar plates see Part II. 


\section{LESSON $\mathrm{X}$}

Vibrio Choleræ Asiaticæ (concluded)-Impression Specimens from Plates

- Comparison of Different Varieties - Curdling Ferment Actinomycosis-Cladothrix-Staining of Tissues embedded in Celloidin.

\section{Cholera (concluded)}

(1) Examine the plates made from the heart's blood of the guinea-pig.

The colonies may be so small that they can only be seen with a magnifying-glass.

Select a typical colony and make a gelatine stab culture from it which should be kept at $22^{\circ} \mathrm{C}$.

Prepare also impression specimens of suitable colonies, and stain them with aniline gentian-violet.

Examine with a high power and $\frac{1}{12}$ in. oil immersion.

(2) Inoculate five gelatine tubes from the five varieties of cholera vibrios supplied (stab cultures).

Place them in the cool incubator at $22^{\circ} \mathrm{C}$.

Compare their mode of growth and rate of liquefaction from day to day. 
(3) Inoculate five milk tubes with the same varieties of cholera vibrios, and keep them at $38.5^{\circ} \mathrm{C}$.

Compare their mode of growth and curdling power from day to day.

(4) Examine the hanging drop cultures of Vibrio choleræ Asiaticæ previously made, and stain them with aniline gentian-violet.

\section{Actinomyces and Cladothrix}

(1) Inoculate an agar-agar tube with Cladothrix nivea and another with Cladothrix asteroides.

Keep them in the cool incubator at $22^{\circ} \mathrm{C}$.

(2) Stain actinomycotic tissue, embedded in celloidin, by Gram's method.

Some of the sections may be previously stained with picrocarmine (vide p. 36).

(a) Place the section on a slide and with a sharp scalpel trim off the celloidin all round.

(b) Dry the specimen with filter-paper.

(c) Filter a little aniline gentian-violet on to the section, and cover it over with a watch-glass, so as to protect it from the dust.

(d) After a quarter to half an hour remove the gentianviolet with blotting-paper (sometimes five minutes is sufficient).

(e) Pour a little of Gram's iodine solution on the section and allow it to act for one to two minutes.

(f) Remove the iodine solution with blotting-paper. 
(g) Pour a little aniline oil over the specimen, which is now quite black, and by alternately tilting first one and then the other end of the slide, allow the oil to flow to and fro over the section. The aniline oil will take up the gentian-violet greedily, and gradually become saturated. When this has taken place, remove the oil with blottingpaper and pour fresh oil over the specimen. Repeat this process till no more stain comes away.

(h) Remove the oil with blotting-paper, and wash the specimen thoroughly with xylol, which must be frequently changed.

(i) Mount in xylol balsam and examine with low and high powers.

It is essential that the whole process be completely carried through with the section on the slide.

Sections of Mycetoma (Madura disease) may be stained in the same manner. 


\section{LESSON XI}

Pyogenic Cocci-Cultures-Hanging Drops-Staining of Pus preserved in Carbolic Acid-Staining of Fresh Pus-Staining of Gonorrhœal Pus.

\section{Pyogenic Cocci}

(1) MAKE a broth culture of the
(a) Streptococcus pyogenes ;
(b) Streptococcus pneumoniæ ;
(c) Streptococcus erysipelatos.

Keep them at $38.5^{\circ}$ C., and compare them from day to day.

(2) Make also agar-agar streak cultures of the same organisms.

Keep them at the same temperature, and compare them from day to day.

(3) Make gelatine stab cultures of the same organisms and keep them at $22^{\circ} \mathrm{C}$.

They all grow slowly in gelatine.

Compare them from day to day.

(4) Prepare agar-agar streak cultures of the Staphylococcus pyogenes albus, aureus, and cereus flavus respectively, and keep them at $38 \cdot 5^{\circ} \mathrm{C}$. 
(5) Prepare gelatine streak cultures of the Staphylococcus pyogenes aureus and cereus flavus respectively, and keep them at $22^{\circ} \mathrm{C}$.

The former will liquefy the gelatine, the latter will not.

(6) Prepare two hanging drop cultures of the Streptococcus pyogenes in the usual manner, and keep them at $38.5^{\circ} \mathrm{C}$.

(7) Prepare films from the condensation water of an agaragar culture of the Streptococcus pyogenes, and stain them

(a) with gentian-violet, fuchsine, or Löffler's methylene-blue ;

(b) by Gram's method.

Mount in xylol balsam and examine with a high power, or $\frac{1}{12}$ in. oil immersion.

(8) Prepare specimens of the pus supplied which contains Streptococci. The pus has been preserved in carbolic acid -(5 per cent solution).

(a) With a platinum loop remove some of the white sediment and place it on a clean cover-glass.

(b) Place another cover-glass on this and rub the two cover-glasses together so as to spread the films uniformly.

(c) Separate the cover-glasses and allow the two films to dry.

(d) When dry, pass the films three times through the flame.

(e) Place the films in a mixture of alcohol and ether (equal parts) for one to two minutes. 
$(f)$ Dry them between blotting-paper and again pass them three times through the flame.

(g) Now stain them in the usual manner with dilute aniline gentian-violet, or by Gram's method.

Mount in xylol balsam and examine with $\frac{1}{12}$ in. oil immersion.

(9) Prepare specimens of the fresh pus supplied which contains Staphylococci.

Prepare thin films in the usual manner, clear them in acetic acid, and stain with eosine and methylene-blue in the following way (vide p. 28) :-

(a) Stain with eosine.

(b) Wash, and dry with filter-paper.

(c) Heat again and stain with Löffler's methylene-blue.

(d) Wash, dry and mount.

Examine with $\frac{1}{12}$ in. oil immersion.

(10) In the same manner prepare and stain specimens of gonorrhœal pus. (Eosine and methylene-blue.)

Examine with $\frac{1}{12}$ in. oil immersion : note the cocci in the cells. 
(3) Stain frozen sections of the following tissues:-

(a) Pyæmic abscesses in cardiac muscle and kidney (Staphylococci from a case of ulcerative endocarditis).

Use Löffler's methylene-blue, or Gram's method.

Place some sections in the eosine methylene-blue mixture (Czinzinski's solution) over night.

(b) Erysipelatous skin.

Use Gram's method, with or without previous picrocarmine staining.

(c) Spleen from a case of pyæmia (Streptococcus).

Use Gram's method without counterstaining.

(d) Croupous pneumonia (lung).

Use Gram's method with or without previous picrocarmine staining.

(e) Lung of mouse dead of infection with the Micrococcus tetragonus.

Stain with the eosine methylene-blue mixture.

In all these cases, whenever Gram's method is used, leave the section in the stain for twenty to thirty minutes, and be careful not to decolourise too much with the alcohol and acid alcohol (vide p. 45).

(4) Fibrin staining in croupous pneumonia (Weigert's method).

To bring out the fibrin network in croupous pneumonia, the following method should be used :-

(a) Place the section on the slide by means of a cigarettepaper. 
(b) Remove the superfluous water with a piece of filterpaper.

(c) Filter a little aniline gentian-violet on to the section, and allow it to act for twenty to thirty minutes.

(d) Remove the aniline gentian-violet with blottingpaper, and wash with 6 per cent saline solution.

(e) Pour a few drops of the following iodine solution on to the section :-

\begin{tabular}{|c|c|c|}
\hline Iodine . & - & 1 gramme \\
\hline Potassium iodide & & 2 grammes \\
\hline Water . & & $100 \mathrm{cc}$ \\
\hline
\end{tabular}

Allow this to act for two to three minutes.

$(f)$ Remove the iodine solution with blotting-paper, and decolourise with a solution of aniline oil (2 parts) and xylol (1 part), in exactly the same manner as described in the case of actinomycosis (vide p. 46).

(g) Wash thoroughly with xylol to remove all trace of the aniline oil.

(h) Mount in xylol balsam and examine with a high power.

The fibrin network and the Pneumococci will be distinctly shown in most cases.

To make the specimen more effective stain the section previously with picrocarmine. 


\section{LESSON XIII}

Bacillus of Typhoid Fever and Bacterium Coli Commune-Cultures and Differences in Mode of Growth-Shake Cultures-Varieties of Bacterium Coli Commune-Staining of Typhoid Spleen-Staining of Capsule of Pneumococcus-Diphtheria-Staining of Diphtheria Bacilli-Staining of Diphtheritic Membrane-Staining of Leprosy Bacillus in the Tissues.

\section{Bacillus of Typhoid Fever and Bacterium Coli Commune}

(1) Prepare cultures of these two organisms for comparison :-

(a) Gelatine streak (at $22.5^{\circ}$ C.)

(b) Potato (at $38.5^{\circ}$ C.)

(c) Agar-agar streak (at $38.5^{\circ} \mathrm{C}$.)

(d) 25 per cent gelatine (at $38.5^{\circ}$ C.)

(e) Broth (at $38.5^{\circ} \mathrm{C}$.)

(f) Milk (at $38 \cdot 5^{\circ} \mathrm{C}$.)

(g) Gelatine containing 4 per cent of a 5 per cent solution of carbolic acid (at $22^{\circ} \mathrm{C}$.)

Compare these cultures from day to day: the Bacterium coli coagulates milk, the typhoid bacillus does not; the typhoid bacillus produces rapid turbidity in 25 per cent gelatine, the 
Bacterium coli much more slowly; in other respects the two organisms in cultures resemble each other closely.

(2) Prepare "shake cultures" of these two organisms.

(a) Melt two ordinary gelatine tubes, and inoculate one with the Bacterium coli and the other with the Bacillus typhosus.

(b) Shake the tubes and allow them to set.

(c) Keep them at $22^{\circ} \mathrm{C}$.

Examine them next day: very active gas formation takes place in the gelatine in the case of the Bacterium coli. commune, none in the case of the typhoid bacillus.

(3) Prepare films of these two organisms in the usual manner.

Stain with aniline gentian-violet for one to two minutes, wash in water, dry and mount.

Examine with $\frac{1}{12}$ in. oil immersion, and compare the two organisms.

There are several varieties of the Bacterium coli commune.

(4) Prepare agar-agar shake cultures of Varieties I., II., and III.

(a) Melt the agar-agar either by placing it in boiling water or by carefully heating the tubes over a small Bunsen flame, and allow the tubes to cool to $40^{\circ} \mathrm{C}$.

(b) Now inoculate them with Varieties I., II., and III. respectively. 
(c) Allow the agar-agar to set, and keep the tubes at $38.5^{\circ} \mathrm{C}$.

Compare them from day to day for gas formation.

(5) Inoculate three milk tubes with the same Varieties, and keep them at $38 \cdot 5^{\circ} \mathrm{C}$.

Examine them from day to day for coagulation.

(Variety I. will coagulate milk, while II. and III. will not.)

(6) Typhoid Spleen.

Stain sections of typhoid spleen with Löffler's methyleneblue or in Czinzinski's fluid, in the usual manner.

Examine with a low and high power, and $\frac{1}{12}$ in. oil immersion.

(7) To stain the capsule of the Pneumococcus prepare the following solution :-

Concentrated alcoholic solution of

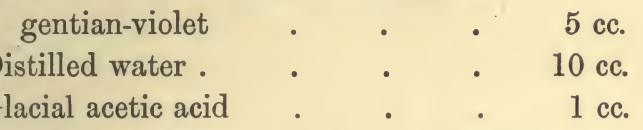

(a) In this solution stain films, prepared from pneumonic sputum, for twenty-four hours.

(b) Then wash in acetic acid (1 per cent) for one or two minutes.

(c) Wash in water.

(d) Dry between blotting-paper.

(e) Mount in xylol balsam.

Examine with $\frac{1}{12}$ in. oil immersion. 
(8) Stain pneumonic sputum kept in carbolic acid by Weigert's method, counterstaining with eosine.

(a) Prepare thin films, as previously described, and after having passed them three times through the flame, wash them in the alcohol-ether mixture (vide p. 48).

(b) Dry the films, and again pass them through the flame.

(c) Stain them with aniline gentian-violet for fifteen to thirty minutes.

(d) Wash them in 6 per cent saline solution.

(e) Place them in Weigert's iodine solution (iodine 1, pot. iod. 2, water 100) for two to three minutes.

(f) Dry thoroughly with filter-paper, and pour a weak alcoholic solution of eosine over the cover-glass, and allow this to act for thirty seconds or less.

(g) Quickly wash in 6 per cent saline solution.

(h) Dry again with filter-paper, and decolourise with the aniline-xylol solution till the specimen is pink.

(i) Wash with xylol and mount in xylol balsam.

Examine with $\frac{1}{12}$ in. oil immersion.

If the sputum has not been kept in carbolic acid, prepare thin films of the fresh viscid sputum, and after passing the dry films three times through the flame, at once proceed to stain with the aniline gentian-violet, and continue as above.

\section{Diphtheria}

(1) Inoculate an agar-agar tube (streak), a serum tube, and a gelatine tube (streak) from an agar-agar culture. 
Keep the first two at $38.5^{\circ} \mathrm{C}$. and the gelatine tube at $22^{\circ} \mathrm{C}$.

(2) Prepare cover-glass specimens from an agar-agar culture and stain with aniline gentian-violet.

(a) Leave them in the stain for five to ten minutes.

(b) Wash thoroughly in water.

(c) Dry and mount.

Examine with $\frac{1}{12}$ in. oil immersion : note the clubbed forms.

(3) Diphtheritic membrane.

Stain sections of diphtheritic membrane with the eosine methylene-blue mixture in the usual manner (vide p. 35$)$.

Examine with low and high powers.

(4) Prepare hanging drop cultures in the usual manner and keep them at $38.5^{\circ} \mathrm{C}$.

Examine them from day to day, so as to study the peculiar forms which the diphtheria bacillus often shows.

\section{Leprosy Tissue}

(1) Stain with picrocarmine, and then according to Weigert's fibrin method (vide p. 51).

Examine with low and high powers and $\frac{1}{12}$ in. oil immersion, and note the arrangement of the bacilli.

(2) Stain a section with a filtered carbol fuchsine solution. 

(a) Leave in the stain for twenty to thirty minutes.
(b) Wash it quickly in 6 per cent saline solution.
(c) Decolourise it in hydrochloric acid (25 per cent) for a few seconds, until the red tint disappears.

(d) Transfer the section to 70 per cent alcohol, and with a needle move it about till no more red is washed out.

(e) Remove the alcohol with 6 per cent saline solution.

$(f)$ Now counterstain the section in Löffler's methyleneblue for one to two minutes.

(g) Wash thoroughly in 6 saline solution.

(h) Dehydrate in absolute alcohol.

(i) Clear in xylol and mount in xylol balsam.

Examine with low and high powers and $\frac{1}{12}$ in. oil immersion. The bacilli are stained red and the tissues blue. 


\section{LESSON XIV}

Tubercle Bacillus-Staining of Fresh Sputum-Staining of Carbolised Sputum-Staining of Frozen Sections-Staining of Paraffin Sections.

(1) Examine the cultures made on the last occasion.

(2) Prepare films of the recent cultures of the Bacillus typhosus and the Bacterium coli commune and stain them with methylene-blue.

(3) Prepare films of the fresh culture of the diphtheria bacillus, and stain them with aniline gentian-violet.

\section{Tubercle Bacillus}

(1) Staining of fresh sputum.

(a) Pour out the sputum in a thin layer into a glass dish, and placing the latter on a dark background select one of the characteristic yellowish particles, and pick it out with a pair of fine-pointed forceps.

(b) Prepare thin films by squeezing the suspected matter between two clean cover-glasses.

(c) Allow them to dry in the air, and then pass them three times through the flame. 
(d) Float the films on a warm carbol fuchsine solution for two to five minutes.

(e) Take one of them out, leaving the other in the stain for subsequent examination if necessary.

$(f)$ Wash it rapidly in water to remove the excess of fuchsiné.

(g) Decolourise it in 25 per cent hydrochloric acid by holding the cover-glass with a pair of forceps and dipping it in the acid just long enough to discharge the red colour.

(h) Wash it immediately in 60 to 70 per cent spirit : at first the red colour reappears ; continue to wash till no more red comes off.

(i) Wash it in water to remove the spirit.

(k) Dry the film between filter-paper and pass it again three times through the flame.

(l) Now stain it in Löffler's methylene-blue (ten to twenty seconds).

(m) Wash it once more in water.

(n) Dry it between filter-paper and again pass it through the flame to dry it.

(o) Mount it in xylol balsam and examine it with an oil immersion.

The tubercle bacilli are stained red on a blue ground.

If no tubercle bacilli be found, the film which was left in the stain should be treated and examined in the same manner.

It is best to keep the water, acid, spirit, and methyleneblue in wide-necked glass-stoppered pots. Holding the 
cover-glass in a pair of forceps, pass it successively from one pot to another, a procedure which saves both time and material. For the solutions are always ready for use and may be employed over and over again.

Repeat the process, using carbol gentian-violet instead of carbol fuchsine, and vesuvine or Bismarck-brown instead of methylene-blue.

Carbol gentian-violet :-

5 per cent carbolic acid . . 10 vols.

Concentrated alcoholic solution of gentian-violet . . . 1 vol.

Leave the specimen in this stain, which must be warmed of course, for at least five minutes.

Be careful not to leave the film in the hydrochloric acid too long.

Vesuvine should be used in concentrated aqueous solution.

The tubercle bacilli are stained blue on a brown ground.

(2) Sputum kept in carbolic acid.

(a) Pour 100 cc. of 5 per cent carbolic acid into a small flask.

(b) Add 10 to 20 cc. of the sputum, unless the latter is watery (vide infra).

(c) Shake vigorously for five minutes, till the sputum is thoroughly disintegrated.

(d) Pour the contents of the flask into a conical urineglass and allow it to stand for twelve to twenty-four hours.

(e) Decant the supernatant fluid. 
$(f)$ With a fine pipette remove a little of the sediment from the extreme depth of the glass and rub it between two cover-glasses.

(g) Dry the films in the air and pass them three times through the flame.

(h) Wash them in a mixture of alcohol and ether (equal parts) for one to three minutes.

(i) Dry them between filter-paper and again pass them three times through the flame.

(k) Stain them with carbol fuchsine or carbol gentianviolet, and proceed in the manner described above.

This is by far the best method for. detecting tubercle bacilli.

If the sputum is watery, pour $100 \mathrm{cc}$. of it into a flask and add 5 cc. of liquefied carbolic acid, shake for five minutes, and then proceed as above.

Urine suspected of containing tubercle bacilli must be treated like watery sputum.

(3) Staining of frozen sections of tuberculous tissues.

(i.) Quick Method.

(a) Place the sections in carbolic acid (5 per cent) for one to two hours. (This may be omitted.)

(b) Stain them in carbol fuchsine or carbol gentian-violet for two hours in an incubator at $38 \cdot 5^{\circ} \mathrm{C}$.

(c) Wash a section rapidly in 6 per cent saline solution or in distilled water.

(d) Now transfer it to 25 per cent hydrochloric acid, and leave it till the colour changes to yellow or brown : a few seconds are sufficient. 
(e) Wash it in 70 per cent spirit, till no more stain comes away.

(f) Again wash the section in distilled water, or 66 per cent saline solution.

(g) Counterstain it, for two minutes in Löffler's methyleneblue if carbol fuchsine was used, or in vesuvine for five minutes if carbol gentian-violet was employed.

(h) Wash it thoroughly in distilled water.

(i) Dehydrate it in absolute alcohol and clear it in xylol.

(k) Transfer the section on to a slide and mount it in xylol balsam.

Examine it with a high power and $\frac{1}{12}$ in. oil immersion.

(ii.) Slow Method.

(a) Place a section in aniline fuchsine or aniline gentianviolet for twelve to twenty-four hours.

(b) Wash it rapidly in water and decolourise it in 25 per cent hydrochloric acid, and then proceed as above.

This method gives extremely good results.

(iii.) Gram's Method.

(a) Stain a few sections first with picrocarmine in the ordinary manner (vide p. 36). hours.

(b) Place them in aniline gentian-violet for twenty-four

(c) Wash a section rapidly in water. 
(d) Place it in Gram's iodine solution for one to two minutes.

(e) Wash it in absolute alcohol for half a minute and then in acid alcohol (3 per cent hydrochloric acid) for not longer than eight to ten seconds.

(f) Again wash it in absolute alcohol till no more stain comes off, and the specimen appears red.

(g) Dehydrate it in absolute alcohol.

(h) Clear it in xylol and mount it in xylol balsam.

Examine it with a high power and $\frac{1}{12}$ in. oil immersion. The bacilli are stained blue on a red ground.

\section{Staining of Paraffin Sections}

(1) Tubercle.

(i.) Slow Method.

(a) Fix a section on a clean cover-glass (if possible without egg albumen or any other fixing medium) and remove the paraffin in the usual manner (heat, xylol, alcohol, and water).

(b) Float the cover-glass, specimen surface downwards, on aniline fuchsine for twenty to twenty-four hours, or on carbol fuchsine for two to three hours.

(c) Now pass it through water, hydrochloric acid, spirit, water, and methylene-blue in exactly the same manner as was done in the case of sputum films.

(d) Wash it in water, dehydrate in absolute alcohol, clear in xylol, and mount. 
Aniline gentian-violet or carbol gentian-violet may be used.

This method gives by far the best results, the sections being thin and well stained, and it should be employed in all cases where good specimens are required. The paraffin must be thoroughly removed.

(ii.) Quick Method.

(a) Fix a section on a clean cover-glass, and remove the paraffin as before.

(b) Now float the specimen on the surface of a warm carbol fuchsine solution for five to ten minutes, and treat it as if it were an ordinary sputum film, except that it must be dehydrated and cleared before mounting.

The section must be as thin as possible, and the carbol fuchsine solution should not be overheated. This method, if combined with quick paraffin embedding, is of great value when a rapid diagnosis is desirable.

(2) Diphtheritic membrane.

(a) Fix a section of the diphtheritic membrane on a cover-glass in the usual manner and remove the paraffin.

(b) Place the cover-glass in the eosine methylene-blue mixture for six to eighteen hours.

(c) Wash it in water till the blue tint has almost disappeared.

(d) Dehydrate it in absolute alcohol, clear, and mount it in xylol balsam. 
Stain another section according to Weigert's fibrin method :

(a) Place it in aniline gentian-violet for half an hour to one hour.

(b) Wash it in 6 per cent saline solution.

(c) Now place it in the iodine solution (iod. 1, pot. iod. 2, and water 100) for two to three minutes.

(d) Wash it again in 6 per cent saline solution.

(e) Decolourise it with the aniline oil and xylol mixture (vide p. 52).

(f) Wash thoroughly with xylol to remove all the aniline oil.

(g) Mount and examine it.

In most cases the fibrin network of the diphtheritic membrane will be beautifully stained, and the bacilli always stand out well.

It is desirable when dealing with paraffin sections, fixed on cover-glasses, to perform the various washings, decolourisations, counterstainings, and clearings in widenecked bottles, holding the cover-glasses in a pair of forceps. 


\section{LESSON XV}

Tubercle Bacillus (concluded)-Films of Pure Cultures-Avian Tuberculosis - Glanders - Tetanus Bacillus - Paraffin Sections of Actinomycosis - Pyæmia - Phagocytosis in the Frog - Inoculation of the Pithed Frog-Hanging Drops of Frog's Lymph-Effect of Temperature on Phagocytosis.

\section{Tubercle Bacilli (pure cultures)}

(1) Mammalian Tubercle.

(a) Prepare cover-glass films from the emulsion supplied.

(b) Stain them with warm carbol fuchsine or carbol gentian-violet, decolourise with 25 per cent hydrochloric acid and 70 per cent alcohol, as in the case of tubercular sputum. Counterstaining is, of course, unnecessary, since no other organisms are present.

(2) Avian Tubercle.

Prepare cover-glass films from the emulsion supplied, and proceed as above.

Examine with $\frac{1}{12}$ in. oil immersion and compare the two kinds of bacilli. 


\section{Glanders-Horse's Lung (paraffin sections)}

(i.) Slow Method.

(a) Fix the sections on clean cover-glasses and dissolve out the paraffin (vide p. 64).

(b) Place them in Löffler's methylene-blue for six to eight hours.

(c) Wash them in distilled water or in 6 per cent saline solution.

(d) Then place them in a solution of tannic acid ( 1 in 10) for four to five hours.

(e) Wash thoroughly in water.

(f) Dehydrate in absolute alcohol.

(g) Clear in xylol and mount.

Examine with a high power and $\frac{1}{12}$ in. oil immersion.

(ii.) Quick Method.

(a) Fix the sections on clean cover-glasses and dissolve out the paraffin as before.

(b) Float the sections for ten to thirty seconds on a solution of carbol methylene-blue (i.e. a saturated solution of methylene-blue in 5 per cent carbolic acid, which must always be filtered before use).

(c) Then wash the sections in water.

(d) Wash them in a 10 per cent solution of tannic acid for half a minute to one minute.

(e) Counterstain in a weak solution of eosine or acid fuchsine in 10 per cent tannic acid, till the sections are red. 
(f) Now wash them in water till they are pink.

(g) Dehydrate in absolute alcohol, clear in xylol and mount.

Examine with a high power and $\frac{1}{12}$ in. oil immersion. The glanders bacilli are blue on a pink background.

\section{Tetanus}

Broth Culture.

(a) Prepare cover-glass films in the usual manner, and pass them three times through the flame.

(b) Clear them in acetic acid, wash and dry, and pass them again through the flame.

(1) Stain some of the films with Löffler's methylene-blue or gentian-violet for two to three minutes.

Examine with $\frac{1}{12}$ in. oil immersion. Observe the drum-stick shape of the spore-bearing bacilli.

(2) Stain other cover-glass films for spores with Löfller's fuchsine and methylene-blue (vide p. 31).

Examine with $\frac{1}{12}$ in. oil immersion. The terminal spores are stained red.

\section{Paraffin Sections of Actinomycosis Hominis}

(1) Weigert's fibrin method.

(a) Fix the sections on clean cover-glasses and dissolve out the paraffin (vide p. 64).

(b) Stain with picrocarmine for half an hour. 
(c) Wash in water, and then place in alcohol for half a minute.

(d) Now float the cover-glass on aniline gentian-violet for five to fifteen minutes, and proceed as described above.

Examine with high and low powers and $\frac{1}{12}$ in. oil immersion. The central mycelium is stained blue, while the clubs, if present, generally remain unstained or are light yellow.

(2) Differential staining of clubs and mycelium.

(a) Fix the sections on clean cover-glasses and dissolve out the paraffin.

(b) Stain in a 2 per cent aqueous solution of rubine for two hours.

(c) Wash in water till the sections are dark red.

(d) Pass quickly through spirit, and then again wash in water till the sections are pink.

(e) Stain in Löffler's methylene-blue for half a minute to one minute.

$(f)$ Wash in alcohol till the sections are almost pink again.

(g) Clear in xylol and mount.

Examine with a high power or $\frac{1}{12}$ in. oil immersion. The clubs are red and the mycelium blue.

Paraffin Sections of Pyæmic Spleen (Streptococcus) stained by Gram's Method

(a) Fix and prepare the sections as before. 
(b) Stain in aniline gentian-violet for half an hour.

(c) Wash in 6 per cent saline solution.

(d) Place in Gram's iodine solution for half a minute.

(e) Again wash in 6 per cent saline solution.

$(f)$ Now wash in absolute alcohol for half a minute.

(g) Quickly pass through 3 per cent hydrochloric acid alcohol (five to ten seconds).

(h) Again wash in absolute alcohol (ten seconds).

(i) Stain with eosine (eosine $\cdot 5$ gramme, 50 per cent alcohol $100 \mathrm{cc}$.) for a second or two.

(k) Wash in absolute alcohol, till the section is pink and apparently quite free from gentian-violet.

(l) Clear in xylol and mount.

Here again, as previously mentioned, the various solutions for washing, etc., are best kept in wide-necked bottles or pots.

\section{Phagocytosis}

A pithed frog has been inoculated under the skin of the thigh, or into the peritoneal cavity, with a few drops of a young broth culture of anthrax bacilli.

Four to six hours later examine its lymph or peritoneal fluid.

(1) Examination in a hanging drop.

(a) Pass a fine capillary tube under the skin of the thigh, or into the peritoneal cavity, and remove some of the fluid, and carefully, avoiding air-bubbles, blow a drop on to 
the centre of a clean cover-glass, sterilised by passing it several times through the flame.

(b) Place the cover-glass, with the drop downwards, on a moistened ring of blotting-paper (vide Fig. 1).

Examine the drop with a high power and with $\frac{1}{12}$ in. oil immersion for phagocytosis (vide footnote p. 10).

(2) Examination of stained specimens.

(a) Spread some of the lymph or peritoneal fluid uni-

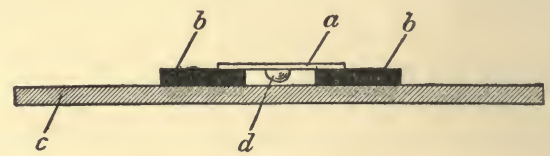

FIG. 1.

$a$, Cover-glass ; $b$, moistened ring of blotting-paper ; $c$, slide ; $d$, drop of lymph or peritoneal fluid.

formly over several cover-glasses, and allow the films to dry in the air.

(b) Clear in acetic acid in the usual manner.

(c) Wash in water, dry, and pass three times through the flame.

(d) Stain with Löffler's methylene-blue, wash, dry and mount.

Examine with $\frac{1}{12}$ in. oil immersion for phagocytosis.

(3) Examination of double-stained specimens.

(a) Prepare films as above.

(b) Stain with a solution of eosine ( 5 gramme in $100 \mathrm{cc}$. of 50 per cent alcohol) for half a minute, without previously clearing in acetic acid. 
(c) Wash in distilled water, and dny between filter paper.

pirtmant

(d) Pass four to six times through the flame.

(e) Stain in Löfller's methylene-blue for about ten seconds.

(f) Wash again, dry and mount.

Examine with a high power and $\frac{1}{12}$ in. oil immersion for phagocytes and eosinophile cells. Observe the relation of the latter to the bacilli.

(4) Local immunity and general infection.

(a) Inoculate a pithed frog under the skin of the thigh with a few drops of a fresh broth culture of Bacillus - anthracis.

(b) To do this, with a fine-pointed pair of scissors snip a small hole in the skin of the ventral surface of the thigh, just above the knee.

(c) With a fine capillary tube blow a small quantity of the broth culture under the skin.

(d) Half an hour later, open the chest of the frog and expose the heart.

(e) With a sterile capillary tube remove some of the heart's blood, blow it over the surface of a sloped gelatine tube, and then distribute the blood uniformly by means of a platinum needle.

(f) Place the tube in the incubator at $22^{\circ} \mathrm{C}$. Twentyfour or forty-eight hours later examine it for colonies of Bacillus anthracis and make impression specimens (vide p. 26). 
Examine with low and high powers. It will be seen that the anthrax bacilli had entered the circulation.

(5) Effect of heat on phagocytosis in the frog.

(a) Inoculate a pithed $^{1}$ frog with a broth culture of Bacillus anthracis as before, and keep it in a moist chamber at $30^{\circ}$ to $35^{\circ} \mathrm{C}$., covering it over with moistened blottingpaper.

(b) Examine the lymph six to eighteen hours later, by means of cover-glass films, stained with methylene-blue in the usual manner.

It will be seen that the bacilli have grown luxuriantly, and that there is no phagocytosis.

Other films should be prepared and stained with eosine and methylene-blue.

It will be seen that there are very few eosinophile cells.

1 In this experiment the cord alone should be destroyed. 


\section{LESSON XVI}

Phagocytosis (concluded)-Effect of Anæsthesia-Phagocytosis in the Hanging Drop-Chemiotaxis.

\section{Phagocytosis (concluded)}

(1) Heated frog.

(a) Remove, with a capillary pipette, some lymph from the inoculated thigh of a frog kept at $30^{\circ}$ to $35^{\circ} \mathrm{C}$. for twelve to eighteen hours (vide p. 74).

Examine it as a hanging drop with a high power, (vide p. 72).

Note that the anthrax bacilli have grown luxuriantly, and that there is no phagocytosis or "eosinophile leucocytosis."

(b) Prepare films of this lymph, dry and stain them

(1) with Löffler's methylene-blue ;

(2) with eosine and methylene-blue.

Examine them with a high power and $\frac{1}{I^{2}}$ in. oil immersion.

(c) Now take the frog out of the incubator and keep it cool for twenty-four to forty-eight hours. 
Then examine its lymph again by means of hanging drops and stained films.

Note the disappearance of the anthrax bacilli, the well-marked phagocytosis and "eosinophile leucocytosis."

(2) Ancesthetised frog.

A frog has been anæsthetised (chloroform-ether mixture), and during the narcosis inoculated under the skin of the right thigh with a virulent culture of anthrax bacilli; and it has then been kept at the ordinary temperature in a moist chamber.

Twenty-four hours later it has been pithed.

Now prepare hanging drops and stained films of its lymph.

Examine them with a high power and $\frac{1}{12}$ in. oil immersion.

Note that the anthrax bacilli have grown well, and that there is no phagocytosis or "eosinophile leucocytosis."

This proves that the immunity of the frog from anthrax does not depend merely on the body temperature.

(3) Phagocytosis in the hanging drop.

(a) Prepare a moist chamber, as described on p. 15.

(b) Pith a healthy frog, and withdraw some of its lymph or peritoneal fluid in the usual manner.

(c) Place a drop of the lymph or fluid on the centre of a sterile cover-glass, avoiding air-bubbles. 
(d) Carefully inoculate this drop from a fresh broth culture of Bacillus anthracis, using a small platinum loop and avoiding over-inoculation.

(e) Place the cover-glass, drop downwards, on the moist ring of filter-paper.

Examine with a high power, or $\frac{1}{12}$ in. oil immersion, using a narrow diaphragm.

Choose a good field for observation, one containing a short chain of bacilli and numerous cells.

Note that the coarsely granular cells attack the bacilli first, and that the phagocytes become active later. Eventually take off the cover-glass, carefully allow the film to dry, and stain it with eosine and methylene-blue in the usual manner.

Examine it with $\frac{1}{12}$ in. oil immersion, and note especially the relation of the eosinophile cells and phagocytes to the bacilli.

\section{Chemiotaxis}

(a) Inject a small quantity of an old broth culture of Bacillus pyocyaneus under the skin of the thigh of a pithed frog.

(b) Four hours later remove some of the lymph and prepare films.

(c) Stain these in the usual manner with eosine and methylene-blue.

Examine with a high power and $\frac{1}{12}$ in. oil immersion. Note the large number of eosinophile cells attracted ("positive chemiotaxis"). 

PART II

\title{
BACTERIOLOGICAL ANALYSIS
}

\author{
LESSONS I-XII
}





\section{LESSONS I AND II}

Cleaning and Sterilisation of Tubes and Flasks - Preparation of Nutrient Media - Beef Broth - Glycerine Broth - Grape-Sugar Broth-Meat Infusion-Gelatine-Grape-Sugar Gelatine-25 per cent Gelatine-Carbolic Acid Gelatine-Peptone Solution-Potato Tubes-Milk Tubes-Agar-Agar-Glycerine Agar-Agar-GrapeSugar Agar-Agar-Serum Tubes.

\section{Cleaning and Sterilisation of Test-Tubes, Flasks, and Beakers}

I. Cleaning of New Test-Tubes.

(1) Roll a little cotton-wool round a strong glass rod and fasten it with a piece of strong thread or string.

(2) Dip the cotton-wool in strong nitric acid, and thoroughly rub the inside of each tube.

(3) Wash the tubes three or four times in water, to remove all trace of the acid.

(4) Allow them to drain, until they are nearly dry.

(5) Rinse each tube with a little absolute alcohol or strong spirit.

(6) Again allow the tubes to drain.

(7) When quite dry, they are ready for sterilisation. 


\section{Cleaning of Test-Tubes which have already} BEEN USED.

(1) Remove the cotton-wool plugs.

(2) Steam the tubes in the steriliser or autoclave for thirty minutes, in order to dissolve the gelatine or agaragar, and to disinfect the tubes.

(3) Pour away the contents of the tubes, and place the empty tubes, upright, in a large sauce-pan containing a weak solution of caustic soda.

(4) Fill each tube with the same caustic soda solution, boil for an hour, and then allow the sauce-pan to cool.

(5) Pour away the caustic soda solution, and wash the tubes three or four times in water.

(6) Now clean each tube with weak nitric acid, using a glass rod to which a little cotton-wool has been fastened.

(7) Wash the tubes repeatedly in water.

(8) Rinse them with a little absolute alcohol, and allow them to drain.

(9) When dry, they are ready for sterilisation.

Very old tubes in which the culture media have dried up must be discarded, since no amount of cleaning will make them fit for use.

III. Cleaning of Flasks and Beakers.

(1) Immediately after use rinse out the flask or beaker with hot water.

(2) With a piece of bent wire, to which a little cottonwool is tied, and which is charged with sapolio, clean the 
inside of the vessel until all dirt, stains, albumen, and fat are removęd.

(3) Wash thoroughly in hot water, and then with nitric acid. If the flasks or beakers are much stained, allow the acid to act for some little time.

(4) Rinse thoroughly with tap water and finally with distilled water.

(5) Allow the vessels to drain; and when dry, they are ready for sterilisation.

IV. Sterilisation of Test - Tubes, Flasks, and BEAKERS.

Place the tubes and vessels when clean in the hot-air steriliser for half an hour to three quarters of an hour, having previously plugged them with cotton-wool.

Sterilisation is completed when the cotton-wool plugs. turn slightly brown.

\section{Preparation of Nutrient Media}

I. Preparation of Beef Broth.

(1) Take one pound of lean beef, remove all fat and connective tissue, and cut it up into small pieces.

(2) Mince it in a sausage machine or chop it up finely.

(3) Add 1000 cc. of distilled water and stir vigorously. Then boil for half an hour to one hour in a large flask or in an enamelled iron pan, which must be well covered up.

(4) Filter, and make the filtrate up to $1000 \mathrm{cc}$. with distilled water. 
(5) Pour it into a large flask containing 5 grammes of sodium chloride and 10 grammes of pure peptope.

(6) Heat this mixture in a water bath or in a steam steriliser, at $100^{\circ} \mathrm{C}$., for an hour, shaking the flask from time to time, so as to prevent the peptone from being charred.

(7) Now neutralise carefully with a concentrated solution of sodium carbonate, making the solution faintly alkaline.

(Should the solution have been made too alkaline, add a little lactate of ammonium, till the proper degree of alkalinity is reached.)

(8) After neutralisation, heat for another half-hour in the water bath or steam steriliser, shaking the flask from time to time as before.

(9) Once more test the reaction, and if unchanged, i.e. if slightly alkaline, filter into a sterilised flask through two layers of Swedish filter-paper.

(10) Sterilise on two successive days in the steamer, for twenty minutes on each occasion. ${ }^{1}$

To fill tubes with broth :-

Take a number of cleaned and sterilised test-tubes.

1 If after the first sterilisation a precipitate appears, or the broth becomes turbid, allow it to settle; then filter again and recommence the sterilisation. If, in spite of a second filtration, the broth does not remain clear, it is advisable to add the white of an egg to the broth, while cool, and to heat it gradually, shaking it from time to time. Heat till all the albumen is precipitated and carried down with the white of egg. Then filter again, and sterilise on three successive occasions. 
Pour some broth into a sterile separating funnel, and allow 5 to $8 \mathrm{cc}$. to run into each tube.

Sterilise these broth-tubes in the steamer, for twenty minutes, on three successive days.

It is advisable during sterilisation to cover up the tubes with a piece of stout tinfoil, so as to prevent the steam from condensing on the cotton-wool plugs.

In any case, when nutrient media are to be sterilised in the steamer, care should be taken that the latter is heated up to $100^{\circ} \mathrm{C}$. before the tubes or flasks are placed in it.

Occasionally after sterilisation a slight precipitate appears in the broth. This will settle gradually, and for ordinary purposes may be disregarded.

\section{Glycerine Broth and Grape-Sugar Broth.}

(a) Glycerine broth.

To every 100 cc. of broth add 4 to 6 cc. of pure glycerine, and shake the flask till they are thoroughly mixed.

Fill sterile test-tubes, and sterilise them in the usual manner.

(b) Grape-sugar broth.

To every 100 cc. of broth add 2 grammes of grape-sugar.

When the sugar is quite dissolved, fill a number of sterile test-tubes, and sterilise them in the usual manner.

Glycerine broth is used for the cultivation of tubercle bacilli, and grape-sugar broth for anaërobic cultivation. 


\section{Meat Infusion.}

(1) Prepare the meat as before (vide p. 83).

(2) To one pound of the minced beef add 1000 cc. of distilled water and allow the mixture to stand in a cool place for twenty-four hours, having previously stirred it vigorously.

(3) Next day strain through muslin, and press as much juice as possible out of the meat.

(4) Make up to 1000 cc. with distilled water.

(5) Pour the strained infusion into a flask, containing 5 grammes of sodium chloride and 10 grammes of peptone, and heat in a water bath or in the steamer for an hour; then proceed exactly as before (vide supra).

(Koch advised the preparation of bouillon with meat infusion. This method, however, has no special advantages and is less convenient, requiring more time.)

IV. Gelatine.

(1) Prepare 1000 cc. of broth (vide supra) and pour it into a large beaker, containing 100 to 120 grammes of sheet gelatine rolled up.

(2) Allow the gelatine to soak for half an hour to one hour.

(3) Place the beaker in a water bath, covering it up with a clean glass plate, and heat the bath slowly and steadily, without letting it reach boiling-point.

(4) When the gelatine has been dissolved neutralise 
carefully, and heat for another half-hour, stirring it from time to time.

(5) Then add the white of an egg, and continue heating till all the albumen is precipitated.

(6) Filter through a hot-water funnel with two layers of moistened filter-paper into sterilised flasks, and sterilise in the steamer on two successive days for twenty minutes. ${ }^{1}$

To fill tubes with gelatine:-

Melt the gelatine and pour it into a sterile separating funnel, and proceed as described for broth-tubes (vide p. 84).

The gelatine tubes must be sterilised in the steamer on two successive days.

After the last sterilisation, place some of the tubes, while still liquid, on a sloped tray and allow the gelatine to set in this manner (for streak cultures). Allow the others to set in an upright position (for stab cultures and plates).

\section{Grape-Sugar Gelatine.}

(1) In every 100 cc. of liquid gelatine dissolve 2 grammes of grape-sugar.

(2) Fill test-tubes, and sterilise on two successive days in the usual manner.

\section{25 Per Cent Gelatine.}

(1) To every $100 \mathrm{cc}$. of liquid 10 per cent gelatine add

${ }^{1}$ Gelatine must not be heated too much nor too long, since by so doing it may lose its property of setting when cold. The gelatine should be quite clear. If it be turbid after sterilisation, this is often due to the fact that it is too alkaline. In such a case add lactate of ammonium, not hydrochloric or nitric acid. 
15 grammes of sheet gelatine, and heat slowly in a beaker, placed in a water bath, till all the gelatine is dissolved.

(2) Neutralise in the usual manner, and again heat for half an hour to one hour.

(3) If necessary, clear with the white of an egg.

(4) Then filter through a hot-water funnel into a sterilised flask.

(5) Fill test-tubes in the usual manner, and sterilise them on two successive days.

Grape-sugar gelatine is employed for anaërobic cultivation, and 25 per cent gelatine is especially useful in the diagnosis between the bacillus of typhoid fever and the Bacterium coli commune.

VII. Carbolic Acid Gelatine.

(1) To every 100 cc. of liquid 10 per cent gelatine add 4 cc. of a 5 per cent solution of pure carbolic acid.

(2) Fill test-tubes in the usual manner, and sterilise them on two successive days.

This gelatine is most useful for the separation of typhoid bacilli or of the Bacterium coli commune.

VIII. Peptone Solution.

(1) To 10 grammes of pure peptone and 5 grammes of sodium chloride add 1000 cc. of distilled water.

(2) Boil for an hour, neutralise carefully in the usual manner, and boil for another half-hour.

(3) Filter into sterilised flasks, and sterilise in the auto- 
clave at $120^{\circ} \mathrm{C}$. for twenty minutes : a single sterilisation suffices, but overheating must be carefully avoided.

(4) Fill test-tubes in the usual manner, and sterilise them in the autoclave.

IX. Potato Tubes.

(1) Take sound large potatoes and scrub them thoroughly.

(2) Cut off the ends, and with a cork-borer bore solid cylinders out of the potatoes.

(3) These cylinders should measure $3 \frac{1}{2}$ to 4 in. in length, and just fit the lumen of the test-tubes.

(4) By means of a diagonal cut divide them in two, and wash them thoroughly in water, which may be slightly alkaline.

(5) Now take sterilised test-tubes, and at the bottom of each place a little moistened cotton-wool. Into each tube drop a piece of potato, with the broad end downwards, and replace the cotton-wool plugs.

(6) Sterilise in the autoclave for half an hour to one hour at $120^{\circ} \mathrm{C}$., covering the tubes up with stout tinfoil. Avoid overheating, otherwise the potatoes will lose their natural white colour and become sodden in appearance.

\section{Milk Tubes.}

(1) Neutralise "separated milk," if it be acid, and fill a number of sterile test-tubes, in the ordinary manner, using a separating funnel.

(2) Sterilise on three successive days in the steamer. 
XI. Agar-Agar.

(1) Weigh out 10 grammes of agar-agar fibre, cut it up finely, and allow it to swell in a very dilute solution of acetic acid (3 to 4 cc. of glacial acetic acid to $500 \mathrm{cc}$. of water) for fifteen minutes.

(2) Drain away the acetic acid.

(3) Wash the agar-agar in distilled water, to remove all trace of acid.

(4) Add the washed agar-agar to 500 cc. of broth and boil : it will dissolve in about fifteen to thirty minutes.

(5) Neutralise carefully - very little sodium carbonate is required-and boil again for a few minutes.

(6) Allow it to cool somewhat; then clear it by adding the white of an egg, and heating it in the steamer for a half to three-quarters of an hour.

(7) Now filter through a hot-water funnel.

To hasten the filtration, if necessary, change the filterpaper frequently, and always keep the unfiltered agar-agar hot (see note p. 92).

(8) Sterilise the filtered agar-agar in thē autoclave for twenty to thirty minutes at $120^{\circ} \mathrm{C}$. : a single sterilisation is sufficient.

Agar-agar prepared in this way will often filter as quickly as gelatine, and is exceedingly clear.

To fill tubes with agar-agar :-

Melt the agar-agar in the autoclave and pour it into a sterile separating funnel, and fill the tubes in the usual manner. 
The tubes afterwards must be sterilised in the autoclave, being at the same time covered up with stout tinfoil.

When the sterilisation is complete, place some tubes on the sloping tray and allow the agar-agar to set on the slant; allow the other tubes to set in an upright position.

The latter may be sloped at any time by again melting the agar-agar in the autoclave and placing the tubes on the sloping tray.

\section{Glycerine Agar-Agar and Grape-Sugar AGAR-Agar.}

(a) Glycerine agar-agar.

(1) To every $100 \mathrm{cc}$. of liquid agar-agar, add 5 to $6 \mathrm{cc}$. of pure glycerine, and mix thoroughly.

(2) Fill test-tubes in the usual manner, and sterilise them in the autoclave.

(3) Some tubes should be allowed to set on the slant, others in an upright position.

(b) Grape-sugar agar-agar.

(1) To every $100 \mathrm{cc}$. of liquid agar-agar add 2 grammes of grape-sugar, and shake till the sugar is dissolved.

(2) Fill test-tubes in the usual manner, and sterilise them in the autoclave at $120^{\circ} \mathrm{C}$.

(3) Allow the agar-agar to set in an upright position.

If the grape-sugar agar-agar is to be used for anaërobic cultivations, the tubes must be filled to two-thirds of their height. 
To prevent the agar-agar from slipping, after having been slanted, allow it to cool and set slowly, and keep the tubes for one or two days at the temperature of the room. ${ }^{1}$

\section{Blood Serum (Lorrain Smith's Method).}

(1) To prepare sloped serum tubes, take $100 \mathrm{cc}$. of fresh ox serum, collected under aseptic precautions.

(2) Add to it 1 to 1.5 cc. of a 10 per cent solution of caustic soda and shake it gently.

(3) Pour the serum into test-tubes and sterilise them, in a sloped position, in the autoclave at $120^{\circ} \mathrm{C}$. for twenty minutes, or in the steamer on three successive days.

By this method almost transparent serum may be readily obtained.

Serum tubes are of especial value in the cultivation of diphtheria bacilli, for diagnostic purposes, and also in the separation of tubercle bacilli.

${ }^{1}$ In preparing agar-agar according to the above method it is not necessary to clear with the white of an egg, though this considerably improves the clearness of the medium. To hasten filtration, in case this should be slow,-as occasionally happens, -instead of changing the papers the hot agar-agar may be first passed through Papier Chardin (Cogit and Co., Paris), and then through two layers of Swedish filterpaper. In any case, before filtering, the agar-agar should be heated up properly. 


\section{LESSON III}

Examination of Water-I. Quantitative Examination by Plate Culture Method and Roll Tubes-Effect of Sunlight on Water.

\section{Quantitative Examination of Water}

CoLLeCT the water to be tested in a sterilised flask.

(a) If tap water, allow it to run for a minute or two before collecting it.

(b) If stagnant water or river water, collect it from varying depths with large sterilised pipettes, and empty the latter into sterilised flasks.

\section{A. TAP WATER.}

(a) Plate culture method.

(1) Collect $100 \mathrm{cc}$. of the water to be examined in a sterilised flask, and shake the latter.

(2) With a sterile graduated pipette, connected with a short india-rubber tube, suck up $25 \mathrm{cc}$. of this water, and add it severally to each of three liquefied gelatine tubes.

To sterilise the pipette, it should be plugged with cotton-wool at its broad end (a), and placed in a test-tube, the mouth of which is also plugged with 
cotton-wool $(b)$. The tube and pipette should then be placed in the hot-air steriliser (vide Fig. 2).

Before using the pipette, fix a thin india-rubber tube to it, without removing the cotton-wool plug.

(3) To other three gelatine tubes add severally $\cdot 1 \mathrm{cc}$. of the water.

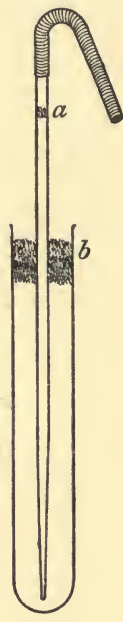

Frg. 2.

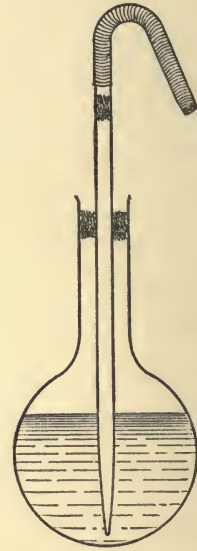

Fig. 3.

(4) Gently shake the gelatine tubes, and prepare plates in the usual manner (vide p. 29).

(5) Keep these in the cool incubator at $22^{\circ} \mathrm{C}$.

If the water to be tested be suspected of containing large numbers of bacilli, smaller quantities, less than $1 \mathrm{cc}$., should be added to the gelatine. ${ }^{1}$

1 The pipette of a Thoma-Zeiss hæmacytometer is well adapted for measuring minute quantities. 
In order to keep the pipette sterile, after it has been used, push it, through a loosely fitting cotton-wool plug, into a flask containing sterile water, which is kept at boiling point over a gas flame (Fig. 3).

Each time before using the pipette, of course, it must be allowed to cool.

Examine the plates from day to day, and count the colonies which appear, in the following manner :-

Cut out a piece of paper, fitting exactly the under surface of the Petri's capsule, and divide it into sixteen equal segments, of which one is painted black (vide Fig. 4). Fix this to the under surface of the capsule, and with a lens or dissecting microscope count the colonies over the black segment. Multiply this number by sixteen, and the result will

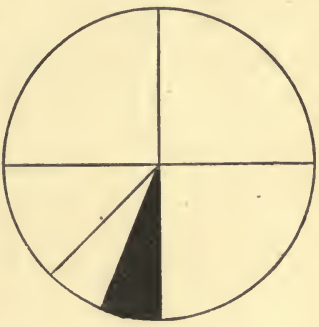

FIG. 4. give the number of organisms in $\cdot 25, \cdot 1 \mathrm{cc}$., or whatever quantity of water was used.

(b) Roll tubes.

(1) Prepare gelatine tubes as before.

(2) Shake them gently, so as to distribute the water and its organisms as uniformly as possible.

(3) With a tube containing boiling water melt a groove in a block of ice. 
(4) Place the gelatine tube, containing the water to be tested, in this groove, keeping it horizontal; and roll it rapidly round and round, until the gelatine sets on the inner surface of the tube in a uniform layer.

(5) Place these roll tubes in the cool incubator at $22^{\circ} \mathrm{C}$. Examine the tubes from day to day, and count the colonies, before liquefaction sets in.

The counting must be done by means of Esmarch's apparatus.

The quantitative examination of water is not of great value, except for the purpose of testing filters, or of comparing different kinds of water, as for instance tap water and distilled water, or of studying the effect of physical or chemical processes on a given sample of water.

B. Distilled Water.

Prepare gelatine tubes and work them up in exactly the same manner as described above, and compare the result of this examination with that of the previous one.

Fresh distilled water contains fewer organisms than tap water.

\section{TANK Water.}

Remove two samples with sterilised pipettes: $(a)$ from the surface; (b) from a given depth (a foot below the surface).

Work these samples up in the manner already described, using, however, not more than $\cdot 1$ or $\cdot 12$ cc.

Compare the results: the surface water will contain more organisms than the water removed from the depth. 
D. Effect of Sunlight on Water.

(1) Fill a tall glass cylinder with tank water, and expose it to active sunlight for several hours, having first removed a sample from the surface by means of a sterile pipette (Sample A).

(2) Having allowed the sun to act on the water for several hours, remove a second sample from the surface, and another from the depth (Samples B and C).

Now work up these three samples in the usual manner, using not more than $\cdot 1$ to $\cdot 12 \mathrm{cc}$.

Compare the results :

Sample A contains most organisms ;

Sample B fewest.

The sun has little or no action on the Bacillus fluorescens liquefaciens, if the latter should be present in the water. 


\section{LESSON IV}

Examination of Water (continued)-II. Qualitative ExaminationExamination of Water for the Bacillus of Typhoid Fever and the Bacterium Coli Commune.

\section{Qualitative Examination of Water}

A. Examination of Water for the Bacillus of Typhoid Fever and the Bacterium Coli Commune.

Two samples of water are supplied (A) and (B), of which (A) contains typhoid bacilli, and (B) a mixture of typhoid bacilli and the Bacterium coli commune.

(1) Take 500 to $1000 \mathrm{cc}$. of each sample and filter through sterile Berkefeld filters into sterilised flasks (vide Fig. 5), which are connected with an air-pump.

The filters should be previously sterilised in the autoclave, the flasks in the hot-air steriliser.

(2) Pour 10 to $20 \mathrm{cc}$. of the filtered water, which is sterile, into a small sterilised beaker, protected with a sterile cotton-wool plug.

(3) Now unscrew the "candle" of the filter.

(4) With a soft sterilised tooth-brush gently scrape the 
"candle" of the filter, so as to get as many as possible of the bacteria from the surface of the candle into the beaker.

(5) The water in the beaker will now be muddy in appearance and contain most of the micro-organisms retained by the filter.

(6) Plug the beaker with cottonwool.

(7) Now melt several carbolic acid gelatine tubes, and to each add, with a sterile pipette, $\cdot 1$ to $\cdot 25 \mathrm{cc}$. of the water containing the bacteria scraped off the filter.

(8) Pour plates in the usual manner, and place them in the cool incubator.

The growth of large numbers of micro-organisms, especially. of

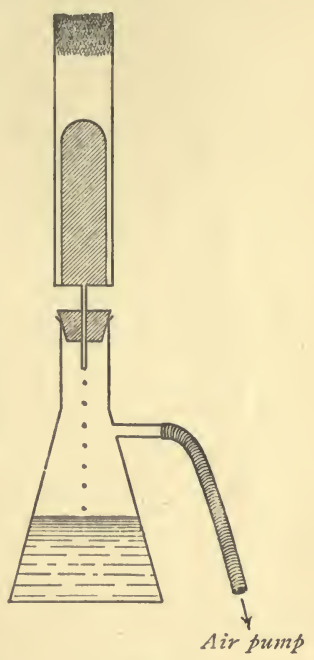

FiG. 5. the liquefying ones, will be inhibited by the carbolic acid contained in the gelatine.

(9) When colonies have appeared, make subcultures of those in any way resembling the bacillus of typhoid fever or the Bacterium coli commune :

(a) gelatine streak cultures;

(b) gelatine shake cultures;

(c) 25 per cent gelatine stab cultures.

Place $(a)$ and $(b)$ in the cool incubator, and $(c)$ in the

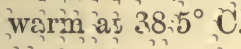


(10) Subsequently select those cultures which apparently consist of typhoid bacilli or the Bacterium coli commune, and confirm the diagnosis by

(a) microscopical examination;

(b) milk cultures (at $38.5^{\circ} \mathrm{C}$.) ;

(c) growth on potatoes (at $38.5^{\circ} \mathrm{C}$.)

For differences between the bacillus of typhoid fever and the Bacterium coli commune vide p. 53. 


\section{LESSON V}

Examination of Water (concluded) - Examination of Water for the Vibrio Choleræe Asiaticæ-Peptone Method-Gruber's MethodAgar- $\Lambda$ gar Plate Method-Gelatine Plate Method.

\section{Qualitative Examination of Water (concluded)}

B. Examination of Water for the Vibrio Cholere ASIATICAE.

A sample of water is supplied containing cholera vibrios (500 cc. of distilled water containing four broth tubes of cholera vibrios).

I. Peptone method.

(1) To separate the vibrios, fill four small sterilised flasks with 50 cc. of 2 per cent peptone solution each.

(2) To these add respectively 50 cc., 40 cc., 25 cc., and $10 \mathrm{cc}$. of the suspected water, and place the flasks in the warm incubator till next morning.

(3) Now examine the surface of the culture fluid for vibrios by means of cover-glass films, which should be stained with carbol fuchsine or aniline gentian-violet (vide p. 40). 
If there be a pellicle on the surface of the culture medium in these flasks, examine it for vibrios.

\section{If vibrios are present-}

(a) Prepare gelatine plates in the usual manner, keep them at $22^{\circ} \mathrm{C}$, and examine them from day to day.

Any suspicious colony should be used for subcultivation :

(1) Under a dissecting microscope fish out the colony with a thin platinum needle, and make gelatine stab cultures and agar-agar streak cultures.

Keep the former at $22^{\circ} \mathrm{C}$. and the latter at $38.5^{\circ} \mathrm{C}$.

Examine from day to day, and prepare cover-glass specimens, which should be stained with carbol fuchsine or aniline gentian-violet (vide p. 40).

(2) From the suspicious colonies start also subcultures in peptone tubes.

Place them in the warm incubator for eighteen to twenty-four hours. At the expiration of this time examine microscopically for vibrios.

Apply also the test for "Cholera Red" to one of the tubes, by adding a few drops of pure concentrated sulphuric acid.

The peptone solution should acquire a markedly red tint.

(b) Inoculate also a series of peptone tubes from the peptone flasks, and keep them at $38.5^{\circ} \mathrm{C}$. for twenty-four hours.

(1) Then test one of these tubes for "Cholera Red." 
(2) Examine another microscopically, and if vibrios are found to be present, prepare gelatine plates and work these up as above.

II. Gruber's method.

(1) Grow cholera vibrios in tubes, containing broth or peptone solution, for twenty-four hours at $38.5^{\circ} \mathrm{C}$.

(2) Then sterilise these tubes by heating them from $60^{\circ} \mathrm{C}$. to $65^{\circ} \mathrm{C}$. for ten minutes in a water bath.

Such tubes should be kept in readiness.

(3) Inoculate four such tubes with $\cdot 1$ to $\cdot 5$ cc. of the suspected water, and place them in the warm incubator for twenty-four hours.

(4) Then test one tube for "Cholera Red," and compare the tint with that obtained from a tube not inoculated with the suspected water: the former should be a deeper red; examine another tube microscopically for vibrios; from the others make gelatine plates, and work them up in the manner described above.

It is often possible to give a definite opinion in from eighteen to forty-eight hours.

\section{Agar-agar plates.}

(1) Liquefy three agar-agar tubes and cool them down to $40^{\circ} \mathrm{C}$. in a water bath.

(2) Inoculate one with $\cdot 25$ to 5 cc. of the suspected water.

(3) From this tube inoculate the second with three platinum loops, and from this second tube the third also with three loops. 
(4) Pour plates in the usual manner, and when the agaragar is firmly set, place them in the warm incubator, lid downwards, so that the condensation water does not collect on the surface of the agar-agar.

(5) Examine the plates next morning microscopically, and make subcultures in gelatine (stab cultures), on agaragar (streak cultures), and in peptone.

Keep the gelatine tubes at $22^{\circ} \mathrm{C}$., the agar-agar and peptone tubes at $38.5^{\circ} \mathrm{C}$.

(6) After twenty-four hours examine microscopically, and test the peptone tubes for "Cholera Red."

This method often yields quick results.

IV. Gelatine plates.

(1) Liquefy three gelatine tubes and inoculate the first with $\cdot 25$ to $\cdot 5$ cc. of the suspected water.

(2) Proceed exactly as in the case of the agar-agar tubes described above.

(3) Prepare three plates and keep them at $22^{\circ} \mathrm{C}$.

(4) Examine these from day to day microscopically, and also make subcultures in the manner stated above.

Unmistakable colonies will be found after thirty-six to forty-eight hours. 


\section{LESSON VI}

Examination of Milk-I. Quantitative Examination by Plate Culture

Method-II. Qualitative Examination-Bacillus of Typhoid Fever

-Bacterium Coli Commune-Streptococcus Pyogenes-Bacillus Diphtheriæ-Tubercle Bacillus

\section{Examination of Milk}

\section{Quantitative Examination.}

As ordinarily obtained, milk always contains microorganisms.

(1) Collect the milk in sterile flasks, having first shaken up the sample supplied.

(2) With a sterile pipette inoculate several liquefied gelatine tubes with varying quantities $(\cdot 1$ cc., $\cdot 2$ cc., $\cdot 3$ cc., $\cdot 5$ cc.)

(3) Prepare gelatine plates, and place them in the cool incubator.

(4) Examine and count the colonies in the same manner as was described on p. 95 .

As in the case of water, so here also, the quantitative examination is of comparatively little use, the qualitative analysis being of much greater importance. 
II. Qualitative Examination.

Five samples of milk are supplied.

Sample A contains Typhoid bacilli.

$\begin{array}{llll} & \text { B } & \text { Bacterium coli commune. } \\ & \text { C } & \text { Streptococcus pyogenes. } \\ & \text { D } & \text { B } & \text { Bacillus diphtheriæ. } \\ & \text { E } & \text { " } & \text { Tubercle bacilli. }\end{array}$

(a) Samples $A$ and $B$ : Bacillus of typhoid fever and the Bacterium coli commune.

Examine for typhoid bacilli and the Bacterium coli commune, in exactly the same manner as was done in the case of water (vide p. 98). It is advisable, however, to dilute the milk with an equal volume of sterile distilled water, so as to facilitate filtration.

(b) Samples $C$ and D: Streptococcus pyogenes and the Bacillus diphtherice.

(1) Use three sloped agar-agar tubes.

(2) Stir up the milk, so as to distribute the suspended organisms uniformly.

(3) Dip a stout, straight platinum needle into the milk.

(4) Now make three parallel streaks on the surface of the first agar-agar tube.

(5) Without dipping the needle again into the milk, make three parallel streaks on the surface of the second agar-agar tube, and also of the third.

(6) Place the three agar-agar tubes in the warm incubator. 
Examine next day.

Pick out the smallest colonies which appear suspicious, and make subcultures on sloped agar-agar, and keep the latter at $38.5^{\circ} \mathrm{C}$. At the same time make a microscopic examination of the suspicious colonies.

Streptococci and diphtheria bacilli, if present in sufficient number, can readily be detected in this manner.

If diphtheria bacilli be suspected, instead of agar-agar, sloped serum, prepared by Lorrain Smith's method, may be used with great advantage (vide p. 92).

(c) Sample $E$ : Tubercle bacillus (Van Ketel's method).

(1) To $50 \mathrm{cc}$. of the suspected milk add $10 \mathrm{cc}$. of strong liquefied carbolic acid or 10 grammes of carbolic acid crystals.

(2) Shake vigorously in a well-corked flask for two to five minutes.

(3) Now pour the carbolised milk into a conical urine glass, and allow it to stand under a glass cover for twentyfour hours.

(4) With a fine capillary pipette remove a little from the deepest layer of the sediment, and prepare films in the usual manner, by rubbing it between two cover-glasses.

(5) Allow the films to dry in the air and pass them three times through the flame.

(6) Now pass the films through a solution of alcohol and ether (equal parts). 
(7) Dry them between folds of blotting-paper and again pass three times through the flame.

(8) Stain in carbol fuchsine, decolourise in hydrochloric acid and 70 per cent alcohol, and counterstain with methylene-blue, as described on p. 60 .

Mount and examine with $\frac{1}{12}$ in. oil immersion for tubercle bacilli. 


\section{LESSON VII}

Examination of Air and Dust-Plate Culture Method-Aspiration through Broth-Filtration through Sugar

\section{Examination of Air and Dust}

\section{Plate Culture Method.}

(1) Prepare three gelatine plates, and expose them to the air at different spots and levels in the room for half an hour, one, and two hours respectively.

(2) Then cover them up with sterile lids, and place them in the cool incubator.

(3) Examine the plates and count the colonies from day to day.

The plates which have been kept nearest the floor, or have been in a draught, will contain most colonies. The number of colonies will also vary with the time of exposure.

Prepare subcultures in gelatine (streak cultures) of the various colonies.

Various kinds of Staphylococci, Sarcinae, and Torulae or Yeasts, or even Cladothrix, probably will be found. 
This is a rough method and of comparatively little use.

\section{Aspiration through Broth.}

Put up an apparatus as shown in the diagram (Fig. 6).

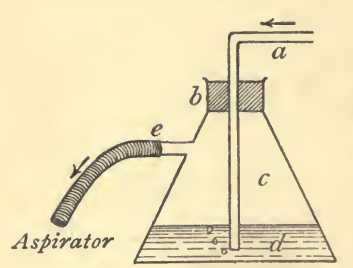

Fig. 6.

(1) Into the flask $(c)$ pour 50 cc. of sterile beef broth; close it with an india-rubber stopper (b), and pass the bent tube $(a)$ through the perforated stopper into the broth.

(2) Now plug the free end of $a$, Bent glass tube 3 to $5 \mathrm{~mm}$. the bent tube and of $e$ with in diameter $; b$, india-rubber stopper; $c$, filtering flask; $d$, broth. cotton-wool, and sterilise the whole apparatus in the autoclave.

(3) When the flask and broth have cooled down, remove the cotton-wool plug at $a$ and slowly aspirate air through the flask by means of the air-pump.

Continue the aspiration for half an hour to one hour.

(4) Now again plug $a$ with sterile cotton-wool.

(5) Liquefy several tubes of gelatine, and to each add .05 to $\cdot 1$ cc. of the broth through which the air has been aspirated, and prepare plates in the ordinary manner.

(6) Keep the plates in a cool incubator; examine them and count the colonies from day to day.

Colonies should be examined, both microscopically and by means of subcultures, as they appear.

\section{Filtration through Sugar.}

(1) Powder a little loaf sugar as finely as possible. 
(2) Draw out a glass tube $(5 \mathrm{~mm}$. in diameter and 12 to $15 \mathrm{~cm}$. in length) at one end and fuse it off here.

(3) Fill the tube with the powdered sugar up to its middle, and plug its open end with two small cotton-wool plugs $\left(p^{1}\right.$ and $\left.p^{2}\right)$, as shown in the diagram (Fig. 7).

(4) Sterilise this tube in the hot-air steriliser at $100^{\circ} \mathrm{C}$. for half an hour to one hour.

(5) When cool remove the plug $p^{2}$, and connect the sugar tube with the aspirating apparatus (Fig. 8).

(6) Fix the sugar tube by means of a clamp $\left(\mathrm{C}^{1}\right.$, Fig. 9), and with a pair of sterile forceps break off its drawn-out end.

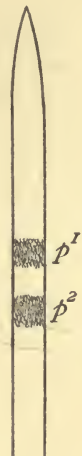

Fig. 7. $p^{1}$ and $p^{2}$, small cotton - wool plugs.

(7) Loosen the clamp of the aspirating apparatus and aspirate by alternately changing the positions

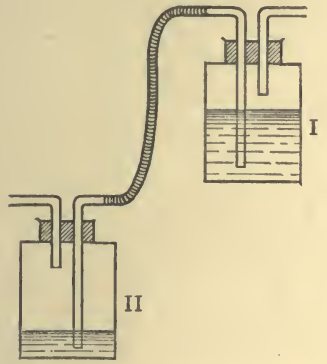

Fig. 8.-Aspirating Apparatus.

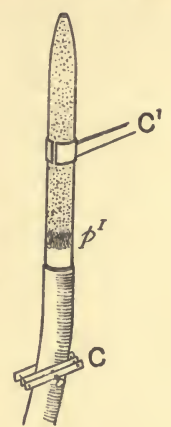

Fig. 9.

of bottles I and II, at the same time altering the connections as required. 
Aspirate for one or two hours, counting the number of aspirations performed, so as to ascertain the volume of air taken and the rapidity of aspiration.

(8) Now remove the sugar tube and shake out the sugar into a flask containing 50 cc. of broth, which of course must be sterile.

(9) Liquefy several gelatine tubes, and add to each from $\cdot 1$ to $\cdot 5 \mathrm{cc}$. of the sugar solution, and pour plates in the usual manner; and work them up as before.

Count the colonies in the plates in the way described on p. 95 . 


\section{LESSON VIII}

Examination of Air and Dust (concluded)-Anaërobic Germs-Examination of Soil - Surface Soil - Tetanus and Malignant Edema Bacilli-Anaërobic Growth in an Exhausted Flask-Anaërobic Growth in Hydrogen-Fractional Separation of Tetanus Bacilli.

\section{Examination of Air and Dust (concluded)}

IV. Anä̈robic Germs in AIr and Dust.

(1) Put up an apparatus as shown in the diagram (Fig. 10).

(2) Place the Wolff bottle (A) in the water bath, which must be kept steadily at $38.5^{\circ} \mathrm{C}$.

(3) Remove the cottonwool plug from $a$, and by means of the aspirator slowly suck air through the warm gelatine for half an hour to one hour.

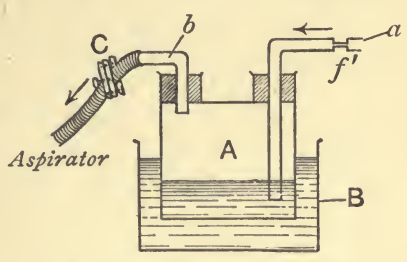

Fig. 10.

A, Sterilised Wolff bottle containing 50 to $100 \mathrm{cc}$. of sterile liquid grapesugar gelatine; B, water bath kept at $38.5^{\circ} \mathrm{C}$.; $a$ and $b$, tubes plugged with cotton-wool ; C, clamp on tube connecting apparatus with aspirator.

(4) Now fuse off the tube at $f^{\prime}$, and aspirate again until the flask (A), which must be kept in the water bath all the time, is exhausted. 
(5) Clamp at $\mathrm{C}$ as tightly as possible, so as to prevent air from entering into the flask (A).

It is advisable to smear all the fittings and joints and the end of the india-rubber tube below $\mathrm{C}$ with hard paraffin.

(6) Place the flask in the cool incubator and watch for the appearance of colonies in the gelatine. If such appear, carefully melt the gelatine at $38^{\circ}$ C. and shake the flask gently, and with a sterile pipette fill each of 5 to 10 tubes with $10 \mathrm{cc}$. of the gelatine.

(7) Prepare roll tubes of them in the ordinary manner. These should be loosely plugged.

(8) Place these tubes in a sterilised, large, wide-necked

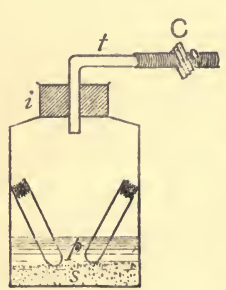

Fic. 11. bottle containing some freshly prepared solution of pyrogallic acid in caustic potash $^{1}$ (Fig. 11). A little moistened sand $(s)$ should previously be placed in the flask, for the tubes to rest on.

(9) Close the wide neck of the flask with a perforated india-rubber stopper (i) and insert a bent tube $(t)$ through the stopper, which must not extend far into the bottle.

(10) Connect the bent tube with the air-pump and exhaust the bottle thoroughly.

(11) When all the air has been exhausted, clamp tightly

1 This is made by using $10 \mathrm{cc}$. of a 10 per cent solution of caustic potash for every gramme of pyrogallic acid required. The caustic potash solution must not be added to the pyrogallic acid until just before closing the flask. 
at $\mathrm{C}$, and place the flask with the roll tubes in the cool incubator for several days.

(12) Then examine the tubes for colonies, and make subcultures in grape-sugar gelatine (stab cultures), and place them in a wide-necked bottle, and proceed as just described.

This method is fairly convenient as a means of separating anaërobic organisms.

\section{Examination of Soil}

A. SURFACE Soll.

(1) Scrape a little earth from the surface of the ground with a sterile knife or spoon, and collect it in a sterile beaker.

(2) Introduce small quantities of it into a number of liquid gelatine tubes with a platinum loop, and gently shake the tubes, so as to distribute the particles of earth as uniformly as possible.

(3) Prepare dilutions in the usual manner.

(4) Make roll tubes as previously described, and place some of these in the cool incubator.

Examine the tubes from day to day, and prepare subcultures from the colonies as they appear.

(5) Place the remaining roll tubes in a large wide-necked bottle as described in the previous section (vide Fig. 10); and having exhausted the flask, place it in the cool incubator.

Examine the tubes from day to day, and prepare subcultures in grape-sugar gelatine (deep stab cultures). 
The latter should be placed in a wide-necked bottle, and this should be exhausted of its air.

Every time the bottle is opened it must be again exhausted.

B. Examination of Soll for Tetanus and MaligNANT GEDEMA BaciLLI.

Two samples of sterilised black garden earth, artificially impregnated with tetanus and malignant œdema bacilli respectively, are supplied.

(a) Growth in an exhausted flask.

(1) Introduce small quantities of each sample into tubes containing liquid grape-sugar gelatine, and gently shake the tubes.

(2) Make several dilutions in the ordinary manner.

(3) Prepare roll tubes.

(4) Place some of these roll tubes in a wide-necked bottle, which must then be exhausted (vide p. 114).

(5) Proceed exactly as described previously (vide p. 114). The tubes must be examined, from day to day, for colonies of tetanus or malignant œdema bacilli.

If any colonies of tetanus or malignant œedema bacilli appear, start subcultures in grape-sugar gelatine (stab cultures), which must be placed in a flask, the air of which is subsequently exhausted.

(b) Growth in hydrogen.

(1) The remaining roll tubes, loosely plugged, should 
be placed in a wide-necked flask or bottle, arranged as shown in Fig. 12.

(2) Connect the flask with a hydrogen apparatus, and allow hydrogen to pass through it for one hour.

The hydrogen should be sent through three wash bottles, containing respectively lead nitrate $(1: 10)$, nitrate of silver $(1: 10)$, and pyrogallic acid (added to 1 per cent caustic potash) solutions, in order to

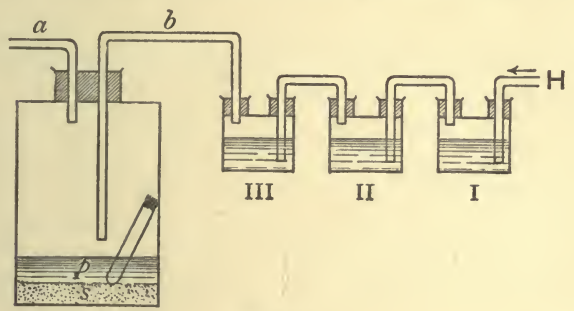

FIG. 12.

I. II. III. Wash bottles; $s$, sand ; $p$, pyrogallic acid and caustic potash.

remove impurities, such as arseniuretted or sulphuretted hydrogen (Fig. 12, I. II. III.)

(3) When the air has been replaced by the hydrogenwhich is best tested by applying a light to the tube $a-$ fuse off at $a$ first, and then also at $b$, and place the flask in the cool incubator.

Examine the tubes from day to day, and work them up as already described, i.e. make subcultures in grape-sugar gelatine (stab cultures).

These must be placed in a wide-necked bottle, through which hydrogen is again passed as before. 
Each time the flask is opened hydrogen must be passed through it again.

(c) Fractional separation of tetanus bacilli.

(1) Introduce small quantities of the earth impregnated with tetanus bacilli into three tubes containing liquid grapesugar agar-agar, and also into three tubes containing liquid grape-sugar gelatine, kept at $60^{\circ} \mathrm{C}$.

(2) Heat them for half an hour at $60^{\circ} \mathrm{C}$., and then allow them to cool.

(3) Now place them in a flask, which must be exhausted in the manner already described (vide p. 114).

(4) Place flask and tubes in the warm incubator for twenty-four hours.

(5) Now heat the tubes again at $60^{\circ} \mathrm{C}$. for half an hour to one hour; and place them in a flask, exhausted of air, in

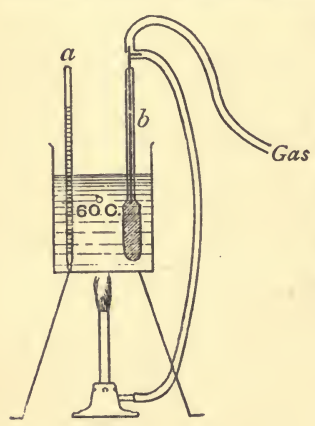

Fig. 13.

$a$, Thermometer ; $b$, thermoregulator. the warm incubator.

(6) Examine the tubes on the second or third day by means of films; and if the cultures appear to be pure, make subcultures in grapesugar agar-agar, and in grape-sugar gelatine (deep stab cultures).

These must be placed in a flask, which then must be exhausted and placed in the warm or cool incubator, as the case may be.

If the cultures, however, are not, or appear not to be, pure, the tubes should be heated once more at $60^{\circ} \mathrm{C}$. for 
half an hour to one hour, as above described. This should be repeated, until the cultures are pure.

The separation of tetanus bacilli without animal inoculations is by no means easy.

The gelatine cultures when pure may be kept in the cool incubator.

To keep the tubes at a steady temperature of $60^{\circ} \mathrm{C}$. arrange a large beaker, filled with water, over a gas flame, and regulate the temperature by means of a thermo-regulator, as shown in the diagram (Fig. 13). 


\section{LESSON IX}

Examination of Decomposing Meat-Aërobic Putrefaction-Anaërobic Putrefaction-How to Examine a Sample of Unsound Meat-How to Examine Meat for Trichina Spiralis-Cysticercus-Psorospermosis-Examination of Ice Cream.

\section{Examination of Decomposing or Diseased Meat}

\section{A. Putrefaction.}

Mince a little fresh meat, and add small quantities of it to five tubes containing sterile nutrient broth.

(a) Aërobic putrefaction.

(1) Place two tubes in the warm incubator.

(2) After three to four days examine the contents of the tubes microscopically by means of cover-glass films, and prepare plates from each in the usual manner.

(3) Work up the colonies as they appear.

Various kinds of Proteus, amongst others the Proteus Zenkeri, Bacillus coli communis, Torulæ, Staphylococci, Sarcinæ, etc., will be found.

(b) Anaërobic putrefaction.

(1) Place two of the tubes in a flask, which should be exhausted of air in the manner already described. 
(2) After three to four days examine these tubes and compare them with the aërobic tubes.

The odour will generally be more marked.

(3) Examine the contents of the tubes microscopically, and then prepare roll tubes from them in the ordinary manner, and place them in a flask exhausted of air, which must be kept in the cool incubator.

(4) Work up the colonies as they appear, and make subcultures in grape-sugar gelatine (deep stab cultures).

(5) Place these in an exhausted flask kept at $22^{\circ} \mathrm{C}$.

Streptococci, Bacillus coli communis, and other bacilli will be found.

(c) Control experiment.

(1) From the last or fifth tube, while fresh, prepare three gelatine plates, and also three gelatine roll tubes, in the usual manner.

(2) Put the plates in the cool incubator, and work up the colonies in the ordinary way.

(3) Keep the roll tubes in the cool incubator in an exhausted flask, and work up the colonies in exactly the same manner as described above.

Compare these results with the previous ones.

(d) Sterilisation and putrefaction.

(1) Take a little fresh, minced meat, and add small quantities of it to broth tubes.

(2) Heat these in the autoclave, in order to sterilise their contents. 
(3) Now keep them at $38.5^{\circ} \mathrm{C}$. for several days.

There will be no smell.

Examine the contents of the tubes microscopically, and make plates.

The latter should remain sterile.

B. How to examine a Sample of Unsound Meat. ${ }^{1}$ effect.

(1) Feed mice or rats with portions of it, and watch the

If they die, make plate cultures from the organs and heart's blood (vide p. 42).

(2) Make an extract in sterile broth or saline solution of the sample supplied, and inoculate mice or other animals subcutaneously.

If they die, make plate cultures as before, and separate the organisms obtained.

(3) Prepare an extract as above, and make plate cultures in the ordinary way, and also roll tubes.

Keep the former aërobically, the latter anaërobically. Separate the various organisms as they appear.

In all cases, the organisms which are separated should be examined as to their virulence, both by means of feeding experiments and by inoculations.

In cases of food poisoning, it has been shown that at times organisms may kill if taken in by the mouth, while they fail to do so if injected subcutaneously. For separation of ptomaïnes and toxines vide p. 172.

1 These experiments can only be performed under a licence and a special certificate. 
C. How to examine Meat for Trichina Spiralis.

(1) Place a small, thin piece of the suspected muscle on a strong glass slide, and press another slide firmly down on to the meat, in order to flatten it out in a uniform layer.

Examine under the low power or with a dissecting microscope : the capsules will be recognised at once as small white points lying between the fibres.

(2) Tease out a few fibres under the low power of a dissecting microscope, and carefully separate the small white bodies.

Mount in Farrant's solution, and examine with low and high powers.

If the capsule of the trichina is calcified, add a few drops of 10 per cent hydrochloric acid in order to dissolve out the lime, wash and drain off the water, and then mount in Farrant's solution.

D. Examination of Cysticercus of Rabbit.

(1) Carefully open the small cyst, and tease out its wall in a drop of water under a dissecting microscope.

(2) Search for the head or scolex, and free it as much as possible from its attachments.

(3) Drain off the superfluous water with a piece of blotting-paper.

(4) Mount in Farrant's solution, applying gentle pressure to the cover-glass, so as to flatten out the head.

Examine under a low power.

Notice the suckers and hooks. 
E. Psorosperms in Rabbit's Liver.

(a) Examination in the unstained condition.

(1) Make a few cuts into the liver and look for white, puriform or caseous masses.

(2) Remove some of the white substance on to a slide, and tease it out carefully.

(3) Add a drop or two of a 10 per cent caustic potash or a little iodine solution, and allow either to act for a few minutes.

(4) Remove the excess of caustic potash or iodine, and then mount the specimen in Farrant's solution.

Examine with low and high powers, using a narrow diaphragm at the same time.

(b) Staining of psorosperms.

(1) Squeeze some of the white matter between two cover-glasses, and when dry pass the films through the flame.

(2) Stain them in Löffler's methylene-blue, or as for tubercle bacilli, in carbol-fuchsine and methylene-blue.

In the latter case the psorosperms will appear red.

\section{Examination of Ice Cream}

(a) Quantitative examination.

(1) Melt some ice cream (100 cc.) in a sterile beaker at $38^{\circ} \mathrm{C}$. and add $500 \mathrm{cc}$. of sterile distilled water to it.

(2) Prepare plates from the mixture, using ordinary gelatine and also carbolic gelatine, inoculating the tubes with quantities varying from $\cdot 1$ to 25 cc. (vide p. 93). 
(3) Keep the plates at the ordinary temperature, count the colonies, and work them up in the ordinary manner, both microscopically and by means of subcultures in gelatine.

(b) Examination for typhoid bacilli and the Bacterium coli commune.

(1) If it is desirable to test ice cream for typhoid bacilli or the Bacterium coli commune, a large quantity of sterile water should be added, for otherwise it will not pass readily through the Berkefeld filter.

(2) Filter through a sterile Berkefeld filter, and with a brush scrape the bacteria from the candle of the filter into 20 to $50 \mathrm{cc}$. of sterile water in a small sterile beaker.

(3) Work this up in the same manner as described on p. 98.

The Bacterium coli commune is frequently found in ordinary street ices. 


\section{LESSON X}

Examination of Antiseptics and Disinfectants-Method of Testing Antiseptics-Methods of Testing Disinfectants-Koch's MethodCarbolic Acid-Mercuric Chloride-Sternberg's Method of Testing Antiseptics-Disinfectant Action of Gases-Sulphur DioxideChlorine-Ammonia.

\section{Examination of Antiseptics and Disinfectants}

A. Method of testing Antiseptics.

Carbolic acid.

(1) Prepare a series of carbolic acid broth tubes (1:100, $1: 200,1: 300,1: 400,1: 500)$.

To tubes containing $10 \mathrm{cc}$. of broth add respectively, with a sterile graduated pipette, $\cdot 1$ cc., $\cdot 05$ cc., .03 cc., .025 cc., .02 cc. of liquid carbolic acid.

(2) Inoculate these five tubes with a platinum-loopful of a pure culture in broth of the bacillus of typhoid fever or blue pus, twenty-four hours old.

Inoculate also an ordinary broth tube with the same organisms, for the purpose of control.

(3) Place the six tubes in the warm incubator.

Examine and compare the tubes from day to day. 
B. Methods of testing Disinfectants.

(i.) Koch's method (modified).

(a) Test the following solutions:

Absolute alcohol;

Mercuric chloride, 1:1000;

Mercuric iodide, $1: 2000$;

Carbolic acid, $1: 20$;

Condy's fluid.

(1) Keep anthrax silk threads ${ }^{1}$ in these solutions for equal periods of time, varying from two to twenty-four hours.

(2) Then take them out of the fluid with sterile forceps or needles.

(3) Wash the threads in sterile water; and do the same with a thread that has not been kept in a disinfecting solution.

(4) Now press the threads into the substance of an agaragar plate, which must be kept at $38.5^{\circ} \mathrm{C}$. Small labels must be fastened on the lid of the Petri's capsule, in order to avoid confusion.

Examine the plate from day to day.

1 Anthrax silk threads are prepared by making, under strictly aseptic precautions, a suspension in broth or 6 per cent saline solution of virulent agar-agar or potato cultures of anthrax bacilli, grown at $38.5^{\circ} \mathrm{C}$. for several days, and known to consist almost entirely of spores.

Sterilised silk threads, abont an inch in length, are allowed to soak in this suspension for half an hour to one hour, are then collected in a sterile capsule, and dried in the warm incubator. 
It will be found that mercuric iodide is more germicidal than mercuric chloride, and that absolute alcohol and Condy's fluid have but little or no effect on the anthrax spores.

(b) Test the following solutions of carbolic acid:

in alcohol, $1: 20$;

in glycerine, $1: 20$;

in water, $1: 20$;

in water, $1: 25$, with 2 to 4 per cent of hydrochloric acid.

Proceed exactly as above, allowing the threads to soak in the solutions for at least four hours.

It will be found that a solution of carbolic acid in weak hydrochloric acid is very efficacious, while solutions in glycerine and alcohol are of little use.

(c) Mercuric chloride $\frac{1}{1000}$.

(1) Keep anthrax threads in this solution for two to four hours.

(2) Now wash some of them in sterile water; wash the others first in a solution of ammonium sulphide and then in water.

(3) Fix the threads on an agar-agar plate, and place the latter in an incubator at $38.5^{\circ} \mathrm{C}$.

Examine the threads from day to day.

Growth will be observed around the threads washed with ammonium sulphide, while around the others there is either no growth at all or very limited growth. 


\section{(ii.) Sternberg's method.}

This method is more convenient than Koch's method for all practical purposes, and the results are more useful, because the conditions of the experiment resemble more closely those of practice.

(1) Take five tubes containing $5 \mathrm{cc}$. of broth each, and inoculate them with the bacillus of typhoid fever.

(2) After twenty-four hours add to four of the tubes respectively 5 cc. of carbolic acid solutions, of the following strengths, $1: 200,1: 100,1: 50,1: 20$. The fifth tube must be left for the purpose of control.

(3) Place the tubes in the warm incubator for two to four hours, or other periods of time.

(4) Then make five subcultures in broth from these five tubes (one or two platinum-loopfuls).

(5) Keep them in the incubator at $38.5^{\circ} \mathrm{C}$. for several days.

Examine the tubes for growth from day to day.

(The carbolic acid solutions must be prepared with sterilised water.)

(C) Disinfectant Action of Gases.

(a) Sulphur dioxide.

(1) Dip strips of sterilised cloth in a fresh broth culture of Staphylococcus pyogenes aureus, place them in a sterile Petri's capsule, and allow them to dry in the warm incubator.

(2) Fix up an apparatus in the fume cupboard to prepare sulphur dioxide, as shown in the diagram (Fig. 14). 
(3) Allow the gas to pass over one of the strips of cloth for one to two hours.

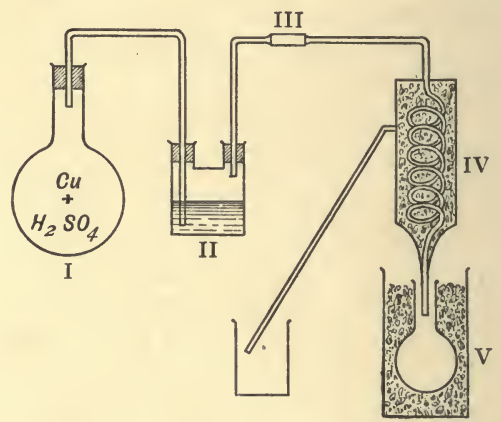

Fia. 14. - Flask I contains some copper and sulphuric acid, which are heated over the flame to prepare the sulphur dioxide; II, Wash bottle containing water; III, Wide glass tube in which the infected strip of cloth is placed; IV, Spiral glass tube surrounded by a freezing mixture of salt and pounded ice; V, Small flask, placed in a beaker containing ice and salt.

(4) Then remove it and drop it into a broth tube, which is to be kept at $38.5^{\circ} \mathrm{C}$. for several days.

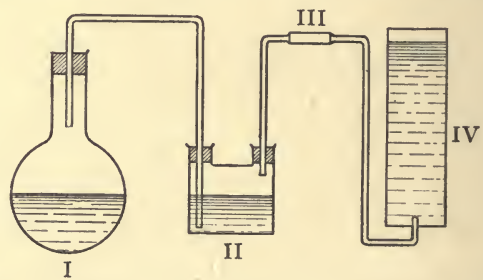

Fig. 15.-I, Flask containing sodium chloride (one part by weight), manganese dioxide (one part by weight), and sulphuric acid and water (two parts by weight of each), which must be very gently heated; II, Wash bottle containing water; III, Wide glass tube containing the infected strip of cloth; IV, Collecting cylinder filled with water.

Examine the contents of the tube for growth from day to day, both microscopically and by means of plates. 
(b) Chlorine.

(1) An apparatus similar to the one just described may be fixed up, as shown in diagram (Fig. 15).

(2) Allow the chlorine to pass over one of the strips of cloth for half an hour to one hour.

(3) Then remove it, and place it in a broth tube to be kept at $38.5^{\circ} \mathrm{C}$.

Examine it for growth from day to day as before.

(c) Ammonia.

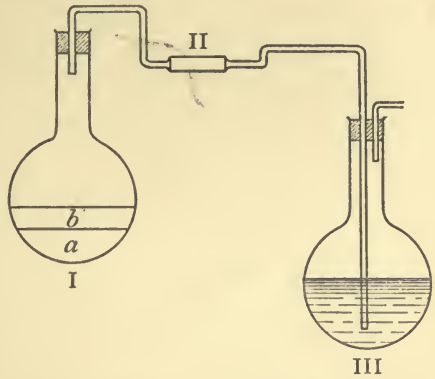

Fig. 16.-I, Flask containing, $a$, mixture of sal-ammoniac (one part by weight) and quicklime (two parts by weight); $b$, a layer of powdered quicklime; the flask with its contents is heated over a Bunsen flame; II, Wide glass tube containing the infected strip; III, Flask containing water to receive the ammonia.

(1) Fix up an apparatus as shown in the accompanying diagram (Fig. 16).

(2) Allow the ammonia to pass over one of the strips of cloth for half an hour to one hour, and proceed as before.

Examine the broth tube for growth from day to day.

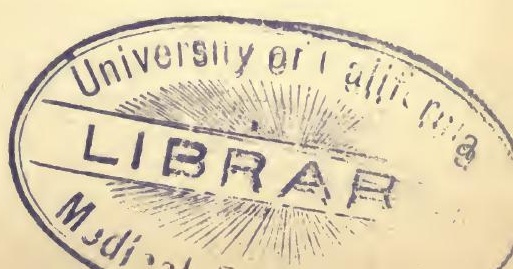




\section{LESSON XI}

Examination of an Animal dead of a Bacterial Disease-Anthrax-

Pyocyaneus Septicæmia-Cholera Asiatica-Rapid Method of Embedding Tissues in Paraffin.

\section{How to Examine an Animal dead of Bacterial Disease}

Three animals are supplied :

(a) mouse inoculated with Bacillus anthracis;

(b) guinea-pig inoculated intraperitoneally with Bacillus pyocyaneus ;

(c) guinea pig-inoculated intraperitoneally with Vibrio choleræ Asiaticæ.

(1) Nail the animal out on a wooden board, thoroughly washed with mercuric chloride $1: 1000$.

(2) Moisten the hairy surface of the animal's abdomen with spirit, and then wash it with mercuric chloride $1: 1000$.

- (3) With a pair of sterilised forceps and a pair of sterilised scissors carefully reflect the skin from the abdomen and chest.

(4) Thoroughly cauterise the exposed surface with a redhot glass rod, and then open the chest and abdominal cavity with sterile instruments. 
(a) Prepare three gelatine plates severally from the spleen, peritoneal fluid, and heart's blood in the ordinary manner, and place them in the cool incubator.

(b) Also make agar-agar streak cultures from the spleen of the mouse, and from the peritoneal fluid of the guineapigs (vide p. 106).

Place these in the warm incubator.

The plates and agar-agar tubes should be worked up in the usual manner.

(c) Prepare also cover-glass specimens of the spleen and peritoneal fluid, and stain them with Löffler's methyleneblue, and examine them with $\frac{1}{12}$ in. oil immersion.

\section{Quick Method of Hardening and of Embedding in Paraffin}

(1) Place small pieces of the mouse's spleen, kidney, liver, and lung in absolute alcohol.

(2) Change the alcohol every half-hour, using always an excess.

(3) After one and a half to two hours place the small pieces of tissue in a small corked bottle containing benzol, till they are transparent (a few minutes).

(4) Now pour off the excess of benzol, so that the pieces are only just covered by it, add a few shavings of paraffin $\left(56^{\circ}\right.$ to $60^{\circ} \mathrm{C}$. melting point), and place the bottle on the paraffin stove, till the paraffin liquefies. 
(5) Transfer the specimens to liquid hard paraffin $\left(56^{\circ}\right.$ to $60^{\circ} \mathrm{C}$.), and allow them to soak for five minutes.

(6) Pour the paraffin and the tissues into a small paper box, and when the paraffin is set, cut out the pieces of tissue, fix them on the rocking microtome, and cut a number of sections, which must be received in warm water at $60^{\circ}$ to $65^{\circ} \mathrm{C}$.

(7) Fix the sections on cover-glasses and dissolve off the paraffin in the usual manner (vide p. 64).

(8) Now stain the specimens fixed on the cover-glasses:

(a) according to Gram's method, as described on pp. 70 and 71 ;

(b) with methylene-blue for five minutes; wash in water for half a minute, then in water acidulated with acetic acid for half a minute, and again in water for half a minute; dehydrate in alcohol, clear and mount (vide p. 35).

Examine the sections under the microscope.

In this manner good specimens can be obtained in less than three hours.

\section{Slow Method of Hardening Tissues}

(1) Pieces of tissues from the mouse also should be placed for a few weeks in Müller's fluid, which must be changed from time to time.

(2) Then wash the specimens in running water, to remove the Müller's fluid. 
(3) Keep the washed tissues in methylated spirit for a week, and then place them in absolute alcohol.

(4) Now embed in paraffin $\left(56^{\circ}\right.$ to $60^{\circ} \mathrm{C}$.) in the ordinary and slower manner, cut and stain.

Examine the sections, and compare them with the others. 


\section{LESSON XII}

Testing of Filters-Filtering through Paper-Filtering through a Berkefeld Filter-The Effect of Use on a Berkefeld Filter.

\section{Testing of Filters}

A. Filtering through Paper.

(1) Take 500 to 1000 cc. of sterile water, and add three broth cultures of the Bacillus prodigiosus (four days old).

(2) Prepare three gelatine plates from this water, before filtering it.

(3) Now filter the water through sterile paper, using a sterile funnel and observing aseptic precautions as strictly as possible.

(4) Prepare three gelatine plates from the filtrate.

Place the two sets of plates in the cool incubator and compare them from day to day, counting the colonies at the same time.

Filter paper arrests only a limited number of organisms.

B. Filtering through a Berkefeld Filter.

(1) Take 1000 cc. of sterile water, and add three broth cultures of the Bacillus prodigiosus as before. 
(2) Filter through a sterile Berkefeld filter into a sterile flask.

(3) Prepare three gelatine plates from the filtrate, and place them in the cool incubator.

Examine them from day to day.

The plates should remain sterile.

C. Effect of Use on a Berkefeld Filter.

(1) Take 1000 cc. of sterile water, and add three broth cultures of the Bacillus prodigiosus as above.

(2) Filter through a sterile Berkefeld filter into a sterile flask.

(3) Pour the filtrate back into the filtering cylinder and filter again.

(4) Repeat this process six times.

(5) Eventually pour the filtrate back into the cylinder and allow the filter to stand for a day.

(6) Repeat the whole process with the same filter on three successive days, without cleaning it out in the meantime.

(7) On the last day fix a fresh sterile flask to the filter, and exhaust it with the air-pump.

(8) From the final filtrate three gelatine plates should be prepared in the usual manner.

Examine them from day to day.

The plates will show colonies of the Bacillus prodigiosus. 
(9) Now clean the "candle" of the filter thoroughly, scrubbing it with a brush, and sterilise the whole apparatus in the autoclave.

(10) When it has cooled down, filter through it $1000 \mathrm{cc}$. of sterile water, to which three broth cultures of the Bacillus prodigiosus have been added.

(11) From the filtrate prepare three gelatine plates, which should be kept in the cool incubator.

Examine them from day to day.

The plates should remain sterile.

The experiment proves that filters at best can do only a limited amount of work, and must be cleansed or sterilised from time to time.

Other filters may be tested in the same manner. 


\title{
PART III
}

\section{BACTERIOLOGICAL CHEMISTRY}

\author{
LESSONS I-X
}





\section{LESSON I}

Preparation of Metabolic Products of Micro-organisms-Heat-Filtration-Combined Heat and Filtration- "Intracellular" PoisonsNitrous Acid in Cholera Cultures.

\section{Preparation of Metabolic Products of Micro-organisms}

(a) Sterilisation by heat.

(1) Inoculate twelve broth tubes with the Bacillus pyocyaneus, and keep them at $38.5^{\circ} \mathrm{C}$. for a week.

(2) Place them in the water bath at $70^{\circ} \mathrm{C}$. for ten to fifteen minutes.

(b) Sterilisation by filtration.

(1) Inoculate several small flasks of broth (containing $50 \mathrm{cc}$ each) with the Bacillus pyocyaneus, and keep them at $38.5^{\circ} \mathrm{C}$. for a week.

(2) Filter the broth cultures through a sterile Berkefeld filter into a sterile flask.

(c) Combined sterilisation by heat and filtration.

(1) Inoculate several small flasks of broth (containing 50 cc. each) with the Bacillus pyocyaneus, and keep them at $38.5^{\circ} \mathrm{C}$. for a week. 
(2) Place them in the water bath at $70^{\circ} \mathrm{C}$. for fifteen minutes.

(3) Filter the sterilised broth cultures through a sterile Berkefeld filter into a sterile flask.

(d) "Intracellular" poisons.

(1) Prepare ten agar-agar streak cultures of the Bacillus prodigiosus, and keep them at $22^{\circ} \mathrm{C}$. for twenty-four to forty-eight hours. The whole surface of the agar-agar should be uniformly inoculated.

(2) To each tube add $10 \mathrm{cc}$. of sterile 6 per cent saline solution, and with a stout platinum needle carefully scrape the culture off the agar-agar into the salt solution, so as to prepare suspensions of the bacilli.

(3) Pour the liquid contents of the tubes into a small flask.

(4) Heat the flask and its contents in a water bath at $65^{\circ}$ to $70^{\circ} \mathrm{C}$. for fifteen minutes.

(e) Filtered intracellular poisons.

Proceed as above, but conclude by filtering the sterilised contents of the flask through a sterile Berkefeld filter into a sterile flask.

In every case the solution, after sterilisation by heat or filtration, must be tested by inoculating from it two agar-agar tubes (streak. cultures). ${ }^{1}$

1 If the agar-agar tubes show any growth, the solutions must be sterilised again, by heat or filtration, as the case may be. 


\section{Test for Nitrous Acid in Cultures}

(1) Filter six broth cultures of the Vibrio choleræ Asiaticæ (two days old) through a small, sterile Berkefeld filter into a sterile flask.

(2) To a portion of the filtrate add a drop or two of hydrochloric acid, and then a solution of the hydrochloric acid salt of meta-phenylenediamine.

The solution will become yellowish red or deep red, according to the amount of nitrous acid (nitrites) present (Griess's reaction).

The red colour is due to the formation of phenylene brown or Bismarck brown.

Test broth cultures of the Proteus vulgaris in the same manner. 


\section{LESSON II}

Proteïnes-Precipitation of Proteïnes by Alcohol-Bacterial Extracts.

\section{Proteïnes (Nencki, Buchner, Römer)}

(a) Bacillus pyocyaneus.

(1) Inoculate twenty agar-agar tubes with the Bacillus pyocyaneus, and keep them at $38.5^{\circ} \mathrm{C}$., until there is a copious growth over the whole surface of the agar-agar.

(2) Into each tube pour 5 cc. of a 5 per cent solution of caustic potash.

(3) With a stout platinum needle carefully scrape the culture off the surface of the agar-agar into the caustic potash.

(4) Collect the caustic potash emulsions thus made in a glass mortar, and gently, but thoroughly, rub the mass up.

(5) Pour the emulsion into a beaker, and digest it in a water bath at $45^{\circ} \mathrm{C}$., till it is liquid.

(6) Filter this through a small Berkefeld filter.

(7) To the filtrate add dilute hydrochloric acid, as long as a precipitate appears. 
(8) Filter through paper, and wash the residue on the filter with the dilute hydrochloric acid, and then with distilled water.

(9) Dissolve the washed residue in the smallest possible quantity of a sterile 5 per cent solution of caustic soda.

Result : alkaline solution of mycoproteïne.

Tests : (1) On neutralising, no precipitate.

(2) Make the solution slightly acid, and add a little salt : a precipitate appears.

(b) Bacillus prodigiosus.

(1) Inoculate twenty potato tubes with the Bacillus prodigiosus, spreading the material thoroughly over the surface of the potatoes; and keep them at $22^{\circ} \mathrm{C}$.

(2) When there is a copious growth, treat the cultures with 5 per cent solution of caustic potash, as described above.

(3) Collect the caustic potash emulsions, and proceed exactly as before.

(c) Precipitation by alcohol.

(1) Inoculate twenty potato tubes with the Bacillus prodigiosus, and keep them at $22^{\circ} \mathrm{C}$.

(2) When there is a copious growth, with a blunt scalpel scrape the cultures off the surface of the potatoes.

(3) Spread the scrapings, in a thin layer, over a plate of glass, and exsiccate rapidly by means of dry heat (up to $100^{\circ} \mathrm{C}$.)

(4) Scrape the dried culture mass off the glass plate, 
and having placed it in a flask, extract it with distilled water by means of shaking.

(5) Filter, and pour the filtrate, drop by drop, into an excess of absolute alcohol : a precipitate appears.

(6) Allow the precipitate to settle. Separate off the alcohol, and dry the residue at $45^{\circ} \mathrm{C}$., to drive off the alcohol.

(d) Bacterial extract.

(1) Inoculate twenty agar-agar tubes with the Bacillus pyocyaneus (streak cultures), and when copious growth has appeared, pour 2 to 3 cc. of a sterile 5 per cent caustic potash solution, or of distilled water, into each tube.

(2) Scrape the cultures off the surface of the agar-agar into the alkaline solution or into the water.

(3) Collect the various emulsions in a flask, and heat for ten minutes in a water bath at $80^{\circ}$ to $100^{\circ} \mathrm{C}$.

(4) Filter through a small Berkefeld filter into a sterilised flask. 


\section{LESSON III}

Bacterial Colouring Matters-Bacillus Pyocyaneus - Bacillus Prodigiosus.

Separation of Bacterial Colouring Matters (Gessard, Andrewes, Fordos)

(a) Bacillus pyocyaneus (Klein-Andrewes).

(1) Inoculate twenty agar-agar tubes (streak cultures) from an actively chromogenic culture, and keep them at $22^{\circ}$ to $30^{\circ} \mathrm{C}$.

(2) When the agar-agar has become dark green, add to each tube 5 to $8 \mathrm{cc}$. of pure chloroform, break up the agaragar with a glass rod, and shake each tube vigorously, till all the blue pigment is dissolved out by the chloroform.

(3) Collect the chloroform extracts in a small flask, and filter through filter-paper moistened with chloroform.

A clear blue solution of pyocyanine is thus obtained, which may be concentrated by slow evaporation in the dark at $38.5^{\circ} \mathrm{C}$.

\section{Reactions}

(1) Evaporate a little of the chloroform solution in a 
porcelain dish at $38.5^{\circ} \mathrm{C}$. in the dark : a crystalline residue of pyocyanine is obtained.

(2) To the blue chloroform solution add dilute sulphuric or hydrochloric acid, drop by drop, and shake.

When thoroughly acidified, the solution turns red.

Allow to settle: the chloroform which sinks to the bottom of the tube is clear and colourless, the supernatant watery solution red.

(3) To this upper red layer add, drop by drop, 10 per cent caustic soda solution and shake.

The blue colour reappears.

Allow to settle: the chloroform which again sinks to the bottom of the tube takes up the blue pyocyanine, while the supernatant watery liquid becomes colourless.

(4) Expose the blue chloroform extract to sunlight.

It soon loses its blue tint and becomes yellowish.

Now add caustic soda solution: the solution assumes a violet tint.

(5) Place four tubes, containing each 5 cc. of the blue chloroform extract, in boxes behind coloured glass (blue, green, yellow, red) and expose them to the action of direct sunlight.

Keep a fifth tube in the dark at $22^{\circ} \mathrm{C}$.

Blue light discharges the blue colour rapidly, while green light preserves it best.

In the dark also it changes slowly. 
(b) Bacillus prodigiosus.

(1) Prepare twenty potato or agar-agar streak cultures of the Bacillus prodigiosus, which must be kept at $22^{\circ} \mathrm{C}$., till the dark red pigment has been formed copiously.

(2) To each tube add 5 to $10 \mathrm{cc}$. of pure ether and shake vigorously, till all the red pigment has been dissolved out.

(3) Collect the ethereal extracts and pour them into a separating funnel : the red ethereal extract will rise to the surface.

(4) Allow it to stand in the dark for twenty-four hours, and then separate the coloured ethereal extract.

(5) Filter this, if necessary, through paper moistened with ether.

A clear red solution is thus obtained, which may be concentrated by slow evaporation in the dark at $38.5^{\circ} \mathrm{C}$.

\section{Reactions}

(1) Evaporate a little in a porcelain dish at $38.5^{\circ} \mathrm{C}$ : a crystalline residue is obtained.

(2) Add a few drops of hydrochloric acid : no change in colour.

(3) Add a few drops of caustic soda or caustic potash: the red colour is discharged on shaking, the upper or ethereal layer turning yellow.

Now add, drop by drop, hydrochloric acid: the red colour will gradually reappear in the upper ethereal layer.

(4) Allow the ethereal red extract to stand in the light : the red colour disappears. 


\section{LESSON IV}

Peptones and Albumoses-Peptones-Albumoses.

\section{Peptones}

Tests for peptones.

Prepare a solution of peptone. ${ }^{1}$

(1) Boil : no coagulation.

(2) Make strongly alkaline with caustic soda, and add a drop of a dilute solution of copper sulphate : a pink colour is produced (biuret reaction).

(3) Add ammonium sulphate to saturation: no precipitate.

(4) Add nitric acid, drop by drop: no precipitate.

(5) Add nitric acid in the presence of chloride of sodium : no precipitate.

(6) Add an excess of picric acid : no precipitate.

(7) Add absolute alcohol : a white precipitate, readily soluble in water.

(8) Add tannic acid : a precipitate, soluble in excess.

(9) Add Millon's reagent, and boil : a red colour.

${ }^{1}$ Peptone puriss. (Adamkiewicz), obtained from E. Merck of Darmstadt. 


\section{Albumoses}

Tests for albumoses.

\section{Solutions of albumoses are supplied.}

(a) Proto-albumose (concentrated solution).

(1) Boil : no coagulation.

(2) Biuret test : pink colour.

(3) Ammonium sulphate: precipitate.

(4) Alcohol : precipitate.

(5) Equal volume of concentrated solution of sodium chloride in acetic acid : precipitate.

Boil : precipitate disappears.

Allow to cool : precipitate reappears.

(6) Concentrated aqueous solution of sodium chloride: precipitate.

(7) Add nitric acid, drop by drop, keeping the solution cool: precipitate, soluble in excess of nitric acid.

Warm gently : precipitate disappears.

Cool again: precipitate reappears.

(8) Excess of picric acid : precipitate.

(9) Neutral copper sulphate : turbidity or precipitate.

(10) Boil with Millon's reagent : red precipitate or colour. (b) Deutero-albumose (concentrated solution).

(1) Boil : no coagulation.

(2) Biuret test : pink colour.

(3) Ammonium sulphate: precipitate.

(4) Alcohol : precipitate.

(5) Equal volume of concentrated solution of sodium chloride in acetic acid : precipitate.

Boil : precipitate disappears.

Allow to cool : precipitate reappears.

(6) Concentrated aqueous solution of sodium chloride : no precipitate.

Now add also acetic acid saturated with sodium chloride : precipitate.

(7) Add nitric acid, drop by drop, keeping the solution cool : no precipitate.

Add a few crystals of sodium chloride first and then nitric acid, drop by drop, keeping the solution cool: precipitate, which disappears on warming and reappears on cooling.

(8) Excess of picric acid: precipitate.

(9) Neutral copper sulphate: precipitate.

(10) Boil with Millon's reagent: red precipitate or colour. 


\section{LESSON V}

Peptones and Albumoses (concluded)-Separation of Albumoses.

\section{Albumoses (concluded)}

SeParation of DEUTERo-ALBUMOSE FROM PRIMARY ALbUMOSES (proto-albumose and hetero-albumose).

Prepare a neutral solution of Witte's "peptonum siccum"1 in the least possible quantity of distilled water.

(1) Add concentrated salt solution: precipitate (I.) (primary albumoses).

(2) Filter.

(3) To filtrate add acetic acid, saturated with salt, as long as a precipitate falls down : precipitate (II.) (mixture of proto- with a little deutero-albumose).

(4) Filter.

(5) Collect precipitates (I.) and (II.) and set them apart (vide infra (14)).

(6) The filtrate, which contains the deutero-albumose, must be placed in a dialysing membrane and be dialysed against distilled water, to remove the acetic acid and the salt.

1 Obtained from E. Merck of Darmstadt. 
A few crystals of thymol should be added, both to the liquid in the membrane and to the water around.

(7) Concentrate the contents of the membrane in vacuo at $37^{\circ} \mathrm{C}$. to $40^{\circ} \mathrm{C}$., having previously added a few thymol crystals.

(8) The concentrated solution should be poured slowly into five to six times its volume of absolute alcohol: precipitate (III.) (deutero-albumose).

(9) Allow the precipitate to stand under the alcohol for several days.

(10) Syphon off the alcohol, and drive off the remainder of the alcohol by keeping the precipitate at $40^{\circ} \mathrm{C}$.

(11) Dissolve the dry residue in the smallest possible quantity of distilled water.

(12) Pour this solution slowly into five to six times its volume of absolute alcohol, and again allow the precipitate which appears to stand under the alcohol for several days.

(13) Separate the alcohol as before, and dry the precipitate in vacuo at $40^{\circ} \mathrm{C}$.

The dry residue is deutero-albumose.

(14) To obtain the hetero-albumose from precipitate (I.), dissolve the latter in the least possible quantity of distilled water.

(15) Dialyse against running water for twelve hours, and then against distilled water for further two to four hours, till all the salt has been removed. (Add thymol crystals as above.)

(16) Pour the dialysed liquid from the membrane into a beaker : if it contains a precipitate-precipitate (IV.) - this is hetero-albumose.

(17) Filter (vide infra (21)). 
(18) Wash the residue on the filter-paper with distilled water, and then repeatedly with absolute alcohol.

(19) Place it under alcohol for several days, in order to dehydrate it completely.

(20) Separate off the alcohol and dry as above.

The dry residue is hetero-albumose.

(21) The filtrate from (17) should be evaporated to a small bulk in vacuo at $40^{\circ} \mathrm{C}$.

(22) To the concentrated liquid add saturated salt solution : precipitate $(\mathrm{V}$.

(23) Filter.

(24) Dry the residue at $40^{\circ} \mathrm{C}$., and redissolve it in the least possible quantity of distilled water.

(25) Dialyse the solution, adding thymol crystals, as described above. $40^{\circ} \mathrm{C}$.

(26) Concentrate the contents of the membrane in vacuo at

(27) Pour the concentrated solution into an excess of absolute alcohol, and allow the precipitate to stand under the alcohol for several days.

(28) Separate off the alcohol, and dry as above.

The dry residue is proto-albumose.

Apply the tests and reactions described in Lesson IV. to concentrated solutions of the albumoses. 


\section{LESSON VI}

Diphtheria Albumoses-Diphtheria Bacilli-Diphtheria Spleen.

\section{Diphtheria Albumoses (Sidney Martin)}

(a) Diphtheria bacilli.

(1) To $200 \mathrm{cc}$. of sterile ox serum add $2.5 \mathrm{cc}$. of a sterile solution of 10 per cent caustic soda and $60 \mathrm{cc}$. of nonpeptonised sterile broth.

This must be done under strictly aseptic conditions.

(2) Fill twenty-six tubes with this solution, using a sterile 10 cc. pipette, and keep the tubes at $56^{\circ} \mathrm{C}$. for five to six days, for several hours each day.

(3) Select the tubes which remain sterile, and inoculate them with virulent diphtheria bacilli.

(4) Keep them at $38.5^{\circ} \mathrm{C}$. for twenty-four to thirty-two days.

(5) Pour the contents of tubes into a large excess of strong methylated spirit (1000 cc.)

(6) Allow the precipitate to settle, and to stand under the spirit for a week or so.

(7) Filter through paper. 
(8) Extract the residue with cold distilled water by means of shaking, until nothing more dissolves out. $40^{\circ} \mathrm{C}$.

(9) Evaporate the watery extract to a small bulk at

(10) Throw this concentrated extract into an excess of absolute alcohol : precipitate of albumoses.

(11) Allow this to stand under absolute alcohol for several days.

Pour off the alcohol.

(a) Residue $=$ albumoses

(b) Alcoholic liquid:

Evaporate to dryness at $40^{\circ} \mathrm{C}$.

Extract residue several times with absolute alcohol :

Deutero-albumose.

(13) Mix (a) and (b) together, and dissolve in the smallest possible quantity of distilled water.

(14) Pour the watery solution into five to six times its volume of absolute alcohol, and allow it to stand for several days.

(15) Pour off the alcohol and dry at $40^{\circ} \mathrm{C}$.

(16) Again dissolve in distilled water.

(17) Pour this solution once more into alcohol, and allow it to stand for several days.

(18) Pour off the alcohol, dry, and redissolve in water, and again pour into absolute alcohol.

(19) Allow the precipitate to stand under absolute alcohol for five to six weeks.

(20) Pour off the alcohol, and dry the residue in vacuo over sulphuric acid. 
Result : a yellowish brown powder, consisting chiefly of deutero-albumose:

(a) insoluble in chloroform, ether, alcohol, ammonium sulphate, and nitric acid, in the presence of sodium chloride;

(b) soluble in cold or boiling water;

(c) giving a marked biuret reaction.

For the separation of the albumoses see page 152 .

(b) Diphtheria spleen.

(1) Mince the spleen of a child dead of diphtheria.

(2) Throw the finely minced mass into a large excess of strong methylated spirit, and allow it to stand for several weeks.

(3) Separate the spirit by means of filtering.

(4) Then proceed exactly as described above (see $(a)$ ). Albumoses, especially deutero-albumose, will be obtained. 


\section{LESSON VII}

Diphtheria Toxine-Action of Magnesium Sulphate and Ammonium Sulphate on Sulphate of Quinine.

\section{Diphtheria Toxine (Uschinsky)}

(1) Prepare a solution of aspartate of sodium:

Water

Glycerine

Sodium chloride

Calcium chloride

Magnesium sulphate

Bi-potassium phosphate

Lactate of ammonium.

Sodium aspartate
1000 cc.

$\cdot 35$ cc.

6 grammes.

$\cdot 1$ gramme.

- $2 \cdot 4$ grammes.

- 2-2.5 grammes.

- 6-7 cc.

- 3.4 grammes.

(2) Mix thoroughly, and heat for half an hour at about $40^{\circ} \mathrm{C}$.

(3) Neutralise with sodium carbonate, and again heat for half an hour.

(4) Filter.

(5) Pour the filtrate into flasks, and sterilise in the steamer in the ordinary manner. 
(6) Inoculate two flasks, containing 100 cc. each, with Bacillus diphtheriæ.

(7) After twenty-four to thirty-two days filter the cultures through a Berkefeld filter.

(8) Evaporate the filtrate down to a small bulk at $40^{\circ} \mathrm{C}$.

\section{Reactions}

(a) Millon's reaction : more or less typical.

(b) Xanthoproteic reaction : more or less typical.

(c) Alcohol : precipitate.

(d) Acetic acid and ferrocyanide of potassium : turbidity after some time.

(e) Phospho-molybdic acid : slight precipitate.

(f) Ammonium sulphate: no precipitate; hence no albumoses present.

(g) No biuret reaction; hence no albumoses or peptones present.

To obtain the toxine in a dry condition, pour the filtrate into an excess absolute alcohol after it has been evaporated down to a small bulk.

Allow the precipitate to settle and to stand under alcohol for a few days.

Separate off the alcohol, and dry the residue at $40^{\circ} \mathrm{C}$.

The readiest method, for all practical purposes, of obtaining diphtheria toxine in solution is to filter virulent broth cultures through a Beikgf edd filtery
as described on p. 141. Jni a

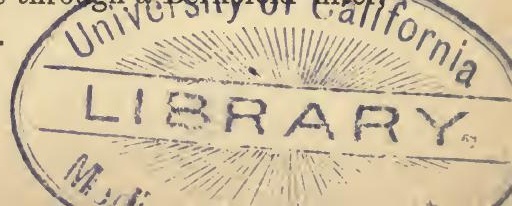




\section{Action of Magnesium Sulphate or Ammonium Sulphate} on Sulphate of Quinine (Duclaux)

(a) Prepare a cold and almost saturated solution of sulphate of quinine.

To it add 10 per cent of finely powdered magnesium sulphate.

A precipitate appears.

(b) Dilute a cold and almost saturated solution of sulphate of quinine with an equal volume of water.

Then add finely powered magnesium sulphate.

A precipitate does not appear until 30 per cent of the salt has been added.

(c) Prepare a cold and saturated solution of sulphate of quinine, and dilute it with one-tenth its volume of water.

(1) Gradually add finely powdered ammonium sulphate, until no more precipitate appears.

Filter.

(2) To the filtrate again add ammonium sulphate, until no more precipitate appears.

Filter.

(3) To the filtrate again add ammonium sulphate, until no more precipitate appears.

The appearance of a precipitate, therefore, depends greatly on the degree of concentration. 


\section{LESSON VIII}

Ferments and Enzymes-Action of Chloroform on Ferments and Enzymes-Action of Heat on Enzymes-Separation of Enzymes (Alcohol Precipitation).

\section{Ferments and Enzymes}

A. Ferment and Enzyme in Yeast.

(1) Prepare a dilute solution of cane sugar.

(2) Test it with Fehling's solution: no reduction of copper.

(3) Pour a little of this sugar solution into two test-tubes, and add a little yeast.

(4) Keep both tubes at $38 \cdot 5^{\circ} \mathrm{C}$.

(5) After a few hours (four to six hours) test one tube with Fehling's solution : marked reduction of copper.

Invertine splits up cane sugar into dextrose and lævulose.

(6) Test the other tube next day with Fehling's solution: no reduction of copper.

Alcohol fermentation has taken place. 
B. Action of Chloroform on Ferments and ENZYMES.

(1) Suspend a little yeast in lukewarm sterile water.

(2) Pour a little of this suspension into two large testtubes.

(3) Shake up the contents of one of these tubes with an equal volume of chloroform for five to six minutes, but leave the other for control purposes (vide infra).

(4) Allow the chloroform to settle to the bottom of the tube.

(5) Add a little of the supernatant liquid to two testtubes, containing a dilute solution of cane sugar.

(6) Keep these tubes at $38.5^{\circ} \mathrm{C}$.

Test one tube with Fehling's solution after four to six hours : marked reduction of copper.

Test the other tube with Fehling's solution after twenty-four hours: marked reduction of copper.

(7) The other tube, containing a little of the yeast suspension, must not be shaken up with chloroform.

(8) Add a little of the original suspension to two tubes, containing a dilute solution of cane sugar.

(9) Keep these tubes at $38.5^{\circ} \mathrm{C}$.

Test one tube with Fehling's solution after four to six hours : marked reduction of copper.

Test the other tube with Fehling's solution after twenty-four hours : no copper reduction. 
Conclusions :

Invertine is an enzyme, and therefore not destroyed by chloroform.

The alcohol-producing ferment is a living ferment, and as such is destroyed by chloroform.

C. Action of Moist Heat on Enzymes.

(1) Prepare a suspension of yeast in lukewarm sterile water.

(2) Shake up a little of it with chloroform as above, and allow the chloroform to settle.

(3) Separate the supernatant liquid, and pour a little of it into two test-tubes.

(4) Heat one of them up to boiling point, leaving the other for control.

(5) Add some of the boiled suspension to two tubes containing a dilute solution of cane sugar, and keep the tubes at $38.5^{\circ} \mathrm{C}$.

Test one tube with Fehling's solution after four to six hours : no reduction of copper and no alcohol.

Test the other tube with Fehling's solution after twenty-four hours : no reduction of copper and no alcohol.

(6) Add some of the suspension in the control tube to two tubes containing a dilute solution of cane sugar, and keep the tubes at $38.5^{\circ} \mathrm{C}$.

Test one tube with Fehling's solution after four to six hours : marked reduction of copper. 
Test the other tube with Fehling's solution after twenty-four hours: marked reduction of copper.

Boiling destroys the enzymes in solution.

D. Separation of Enzymes.

(a) Precipitation by alcohol (Barth).

(1) Fresh yeast is dried at the ordinary temperature in vacuo over sulphuric acid (a fairly large quantity of yeast must be used).

(2) Rub up the dried yeast into a fine powder.

(3) Dry the powder in the hot-air chamber at $100^{\circ} \mathrm{C}$. for six hours.

(4) Allow it to cool, and when it is quite cold add distilled water, so as to convert it into a thin mess.

(5) Let this suspension stand and settle at $40^{\circ} \mathrm{C}$. for twelve hours.

(6) Decant the supernatant water, and filter it till it is clear.

(7) Pour the filtrate into five to six times its volume of 90 per cent spirit : a precipitate appears.

(8) Allow the precipitate to settle, and filter at once.

(9) Wash the residue on the filter-paper with absolute alcohol.

(10) Remove the alcohol by means of pressure, and shake the residue up with water.

(11) Again filter, and precipitate the filtrate with alcohol. 
(12) Filter, and wash the residue on the paper with absolute alcohol as before.

(13) Remove the alcohol by means of pressure and by drying at $40^{\circ} \mathrm{C}$.

(14) Complete the drying in vacuo over sulphuric acid.

The resulting powder is invertine.

Instead of using dried yeast, a suspension of yeast in lukewarm water may be shaken up with an equal volume of chloroform in a shaking machine for twenty to thirty minutes.

The chloroform should be allowed to settle, and the supernatant watery suspension poured into three times its volume of 90 per cent spirit.

The resulting precipitate must be treated as described above under (8) to (14).

\section{Tests}

(1) Add a little of the powder to two test-tubes containing a dilute solution of cane sugar, and keep the tubes at $38.5^{\circ} \mathrm{C}$.

Test one tube with Fehling's solution after four to six hours : reduction of copper, no alcohol.

Test the other tube with Fehling's solution after twenty-four hours : reduction of copper, no alcohol.

(2) Heat some of the dry powder to $100^{\circ} \mathrm{C}$. for a few minutes, and then add a little of it to two test-tubes containing a dilute solution of cane sugar. Keep the tubes at $38 \cdot 5^{\circ}$. 
Test them as before with Fehling's solution, after four to six hours, and again after twenty-four hours : reduction of copper, no alcohol.

(3) Heat some of the dry powder at $130^{\circ}$ or $135^{\circ} \mathrm{C}$. for fifteen minutes, and repeat the previous experiment.

On testing with Fehling's solution, no reduction of copper or alcohol.

Conclusions :

Enzymes in solution are readily rendered inert by heat.

Enzymes in a solid and dry condition are destroyed with difficulty by heat. 


\section{LESSON IX}

Ferments and Enzymes(concluded) - Precipitation by Calcium Phosphate -Proteolytic Enzymes.

\section{Ferments and Enzymes (concluded)}

D. Separation of Enzymes (concluded).

(b) Precipitation by calcium phosphate (Von Brücke).

(1) Prepare a suspension of fresh yeast in dilute phosphoric acid (a fairly large quantity of yeast must be used).

(2) Keep this suspension at $38.5^{\circ} \mathrm{C}$. for five to six days.

(3) Now neutralise it with lime-water: precipitate of calcium phosphate, which carries the enzyme down with it.

(4) Filter, and thoroughly wash the precipitate with distilled water.

(5) Dissolve the precipitate in the least possible amount of water, acidulated with hydrochloric acid.

(6) Dialyse the resulting acid solution, replacing the hydrochloric acid from time to time, so as to keep the solution within the membrane acid.

(7) When all the calcium phosphate, and eventually the hydrochloric acid, have been removed by dialysis, pour the 
contents of the membrane, slowly and gradually, into an excess of alcohol : a precipitate appears.

(8) Filter at once, and collect the residue.

(9) Dry the latter, and remove the alcohol by means of heat at $40^{\circ} \mathrm{C}$., and complete the drying in vacuo over sulphuric acid.

Test the resulting powder (invertine) as described above (vide p. 165).

E. Proteolytic (tryptic) Enzymes of Micro-organisMS Which LIqUefY Gelatine (Fermi).

(1) Prepare thirty roll tubes severally of

(a) Bacillus prodigiosus;

(b) Bacillus pyocyaneus;

(c) Vibrio Finkler-Prior;

(d) Vibrio choleræ Asiaticæ.

(2) When the gelatine is liquefied collect the contents of the tubes in small flasks.

(3) To 200 cc. of liquefied gelatine culture add 200 cc. of dilute alcohol, viz. :

(a) in the case of the Bacillus prodigiosus, 65 per cent alcohol;

(b) in the case of the Bacillus pyocyaneus, 75 per cent alcohol ;

(c) in the case of the Vibrio Finkler-Prior, 70 per cent alcohol ;

$(d)$ in the case of the Vibrio choleræ Asiaticæ, 65 per cent alcohol. 
(4) Allow to stand for twenty-four hours, and then filter through paper.

(5) To each of the four filtrates add absolute alcohol in excess : a precipitate appears.

(6) Filter, and dry the four precipitates at $40^{\circ} \mathrm{C}$. in the ordinary manner.

(7) Dissolve the dry residues severally in $100 \mathrm{cc}$. of saturated thymol water: enzyme solutions.

\section{Tests for tryptic enzymes}

(1) To four tubes of thymol gelatine (7 grammes of gelatine and $100 \mathrm{cc}$. of thymol water) severally add $5 \mathrm{cc}$. of the ferment solutions.

Allow the tubes to stand at $15^{\circ} \mathrm{C}$.

The gelatine will be liquefied after a time.

(2) Place $\cdot 5$ gramme of dried fibrin in two test-tubes.

Add to one of them $5 \mathrm{cc}$. of the enzyme solution of the Bacillus prodigiosus, and to the other $5 \mathrm{cc}$. of the enzyme solution of the Vibrio Finkler-Prior.

Examine the tubes after eight hours: the fibrin is almost entirely liquefied in the case of the former enzyme, and partially so in the case of the latter.

F. Tryptic Enzymes of Bacillus prodigiosus or Bacillus pyocyaneus (Fermi).

(1) Grow the Bacillus prodigiosus or the Bacillus pyocyaneus in the following solution : 
Ammonium phosphate

Bi-potassium phosphate

Magnesium sulphate .

Glycerine.

Distilled water .
10 grammes.

1 gramme.

2 gramme.

40-50 cc.

$1000 \mathrm{cc}$.

(2) Two to three litres should be prepared and distributed in twenty to thirty small flasks.

(3) Allow the inoculated flasks to stand at $30^{\circ} \mathrm{C}$. for a week.

(4) Filter the cultures through a Berkefeld filter.

(5) Evaporate the filtrate to a small bulk in a vacuum pan.

(6) Pour this concentrated solution into eight to ten times its volume of absolute alcohol : a precipitate appears.

(7) Filter through a paper filter, and thoroughly wash the residue on the paper with absolute alcohol.

(8) Redissolve the washed residue in the least possible quantity of distilled water.

(9) Dialyse against distilled water or running water for twelve to twenty-four hours.

(10) Now evaporate the dialysed solution to a small bulk in a vacuum pan.

(11) Pour the concentrated solution into eight to ten times its volume of absolute alcohol as before.

(12) Filter through a paper filter, and wash the residue with absolute alcohol.

(13) Collect the washed residue in a small glass dish, and drive off the alcohol by heating it to $45^{\circ} \mathrm{C}$. 
To test the tryptic action of the dry powder, dissolve a little of it in a small quantity of a 5 to 1 per cent sterilised carbolic acid solution and add it to a tube of carbol gelatine ( 5 to 7 grammes of gelatine to 100 cc. of a 1 to 2 per cent solution of carbolic acid).

The gelatine will be liquefied. 


\title{
LESSON X
}

\author{
Ptomaïnes-Cadaverine-Putrescine
}

\section{Ptomaines}

Cadaverine and Putrescine (Udransky and Baumann).

(1) Allow a solution of white of egg to putrefy for several days.

(2) Distil to a small volume: indol, scatol, and phenol pass over in the distillate, and are disregarded.

(3) Filter the residue.

(4) To the filtrate add an equal volume of a 10 per cent solution of caustic soda.

(5) Shake, and gradually add, drop by drop, a solution of benzoyl chloride : a crystalline precipitate appears.

(6) Allow to stand for several days.

(7)

Filter.

(a) Strongly acidulate the turbid filtrate with sulphuric acid (benzoic acid passes off).

(b) Shake up with three times its volume of ether and (a) The residue must be digested in spirit.

(b) Filter and evaporate the filtrate to a small bulk. 
(c) Separate the ethereal extract.

(d) Repeat this three times and collect the ethereal extracts.

(e) Distil off the ether.

$(f)$ Neutralise the residue, before it sets, with 12 per cent caustic soda : turbid liquid.

(g) Mix with three to four times its volume of 12 per cent caustic soda, and keep in the cold for twelve to twenty-four hours.

(h) Remove the liquid from the crystals which have formed and wash the crystalline residue with cold caustic soda.

(i) Now wash thoroughly with water.

(k) Dissolve the crystals in warm spirit.

(l) Add a large excess of water: the crystals are reprecipitated.

$(m)$ Allow to settle and filter.

(8) Mix the two crystalline precipitates, and dissolve them in the least possible quantity of warm alcohol.

(9) Pour the alcoholic solution into twenty times its volume of ether.

(10) Shake, and allow to crystallise.

(11) Filter : residue consists of the benzoyl compound of tetramethylene-diamine or putrescine.

(12) Filtrate : distil off the alcohol and ether.

(13) Crystals appear: benzoyl compound of pentamethylene diamine or cadaverine. (c) Pour this into thirty times

(d) Allow to stand for several

(e) Filter.

(f) Wash crystalline residue with water, until the washings are quite

(g) Press out as much of the water as possible.

(h) Dissolve the crystalline residue in the least possible quantity of absolute alcohol.

(i) Pour this solution into a

(k) Filter again and redissolve the residue in the least possible quantity of warm alcohol : crystalline precipitate will appear. 
(14) The crystals of these two substances may be purified by dissolving them in spirit and allowing them to crystallise out again.

\section{Tetramethylene-diamine (putrescine).}

(a) Dissolve crystals in a solution of equal volumes of alcohol and concentrated hydrochloric acid.

(b) Heat in water bath at $45^{\circ} \mathrm{C}$. for twelve hours.

(c) Dilute with water, till no more precipitate appears.

(d) Eilter.

(e) Filtrate shake with ether, and separate the ethereal extract.

(f) Slowly evaporate the ethereal extract: crystalline mass, with difficulty soluble in alcohol= hydrochlorate of putrescine.

(g) To a concentrated aqueous solution add an alcoholic solution of platinum chloride: crystalline double salt.

(h) Dissolve in hot water and recrystallise.

\section{Pentamethylene-diamine (cadaverine).}

(a) Dissolve crystals in a solution of equal volumes of alcohol and concentrated hydrochloric acid.

(b) Heat in water bath at $45^{\circ} \mathrm{C}$. for two days.

(c) Dilute with water, till no more precipitate appears.

(d) Filter.

(e) Filtrate shake with ether, and separate the ethereal extract.

$(f)$ Slowly evaporate the ethereal extract : crystalline mass, soluble in water, not readily soluble in alcohol $=$ hydrochlorate of cadaverine.

(g) To a concentrated spirit solution add an alcoholic solution of platinum chloride : crystalline double salt.

(h) Dissolve in hot water and recrystallise.

Cultures of cholera and Finkler-Prior vibrios may be examined for ptomaïnes in the same manner. 


\section{INDEX}

ACETIC acid for clearing, 9 decolourising, 10

Acid alcohol for decolourising, 30, 37

Actinomyces, 45, 46, 69, 70

Actinomycosis, 45, 46, 69, 70 paraffin sections, 69 staining of mycelium, 69

clubs and mycelium, 70

Agar-agar, preparation of nutrient, 90

plates, 103, 104

sterilisation of, 91

to fill tubes with, 90

Air, examination of, 108-115

anaërobic germs, 113-115

Albumoses, 151-155

diphtheria, 155-157

separation of, 152

tests for, 151

Ammonia, disinfectant action of, 131

Anaërobic organisms, 113-119

in air and dust, 113-115

in soil, 115-119

cultivation of, 113-118

separation of, 118, 119

Anaërobic putrefaction, 120, 121

Andrewes, 147

Aniline dyes, 9

fuchsine, 30

gentian violet, 18

water, 18

xylol, 52
Anthrax bacillus, 21-30, 32, 34 $37,71-77$

asporogenous cultivation, 22

attenuation, $23,25,26$

cultivation of, 21

examination of animal dead of, 132

frozen sections, 35

gelatine plates of, 29,34

hanging drop, 22, 24, 25

immunity of frog, 73, 74

impression specimens, 26,34

in tissues, 27,35

litmus agar-agar, 25, 26, 29

phagocytosis, 71-77

production of acid by, 22

silk threads, 127

staining of, 23,24

spores of, 30,32

Antiseptics, method of testing (carbolic acid), 126

Aspartate of sodium, 158

Aspergillus niger, cultivation of, 6 examination of, 13

Aspirator, 110, 111

Avian tubercle, 67

BACILlus anthracis (vide Anthrax) coli communis (vide Bacterium coli commune)

diphtheriæ (vide Diphtheria)

filamentosus, $8,14,17,32$

fluorescens, 6, 21, 97

hay, 33

lepræ (vide Leprosy) 
Bacillus mallei, 68, 69

megatherium, 32

of glanders, 68,69

of tetanus, $69,116-118$

of typhoid fever (vide Typhoid)

prodigiosus, $5,12,21,126,136$ $138,142,145,149,168-$ 170

pyocyaneus, 4, 132, 141, 142, $144,146,147,168-170$

subtilis, 33

tuberculosis (vide Tubercle)

typhosus (vide Typhoid)

Bacterial colouring matter, 147

enzymes, 167

extracts, 146

ferments, 167

metabolic products, 141

poisons, 141

products, 141

toxines, 141 (vide Albumoses, Ptomaïnes, Proteïnes)

Bacterium coli commune, 21, 53$55,59,98-100,106,120$, 124

cultivation of, 53

curdling ferment, 21,55

gas formation by, 54

in ice cream, 124, 125

in meat, 120,121

in milk, 106

in water, $98-100$

shake cultures, 54

staining of, 54

varieties of, 54

Baumann, 172

Beakers, cleaning of, 82 sterilisation of, 83

Beef broth, preparation of, 83 sterilisation of, 85

to fill tubes with, 84

Berkefeld filter (vide Filter)

Blood serum, 153

(Lorrain Smith), preparation of, 92

sterilisation of, 92

Broth (vide Beef broth)

Brücke, Von, 167
Cadaverine, 172-174

hydrochlorate of, 174

Calcium phosphate and ferments, 167

Carbol fuchsine, 30

Carbol gelatine, 88, 171

Carbol gentian-violet, 61

Carbolised sputum, pneumonic, 56 tubercular, 61

Celloidin sections, 45,46

Chemiotaxis, 77

Chlorine, disinfectant action of, 131

Chloroform, action on invertine, 162

enzymes, 162

ferments, 162

Cholera, $37-45$

action of sunlight on, 43

cultivations, 37

enzymes, 168

examination of animal dead of, $41,42,132$

examination of water, 101-104

flagella, 38,39

hanging drop cultures, 41,45

nitrous acid in cultures, 143

plates, 42,44

red, 143

staining of vibrio, 40

varieties, 44,45

Chromogenic organisms, 3, 147 (vide Bacillus prodigiosus and Bacillus pyocyaneus)

Cladothrix asteroides, 45

nivea, 45

Cleaning of beakers, 82

flasks, 82

new test-tubes, 81

used test-tubes, 82

Clearing of films with acetic acid, 9

tissues, 35

frozen sections, 35

with aniline xylol, 52

Colonies, counting of, 95,96

Colouring matters, bacterial, 147

Counting of colonies, 95,96 
Cover-glass specimens (vide Films) cleaning of, 38

Cream, ice, 124, 125

Cultivations, agar-agar, 3,7

broth, 8

gelatine, 5,7

potato, 6

in hanging drops, 14, 17

in plates, 29, 103

Curdling ferment, 21, 45

Cysticercus, 123

Czinzinski's solution, 35

Dedtero-albumose, 152 (vide Albumoses)

Diphtheria albumoses, 155-157 separation from cultures, 155 , 157

separation from spleen, 157

cultivations, 57

films, 59

hanging drop, 57

in frozen sections, 57

in milk, 106

in paraffin, 65,66

meinbrane, 65,66

staining of, 57

toxines, 158

Disinfectant action of gases, 129-131

sulphur dioxide, 129

ammonia, 131

chlorine, 131

Disinfectants, method of testing, 127-131

Koch's method, 127

Sternberg's method, 128

Distilled water, examination of, 96

Double staining, with eosine and methylene-blue, 28, 36, 72, 73,75

with Czinzinski's fluid, 35

with methylene-blue and picrocarmine, 36

(vide also Gram's method)

Duclaux, 160

Dust (vide Air) -

EMBEDDING in paraffin, 133,135
Endocarditis, ulcerative, 57

Enzymes, 161-171

action of chloroform on, 162

action of moist heat on, 163

precipitation by calcium phosphate, 167

proteolytic, 168

separation of, 164

tests for, 165

tryptic, $169-171$

Eosine, 28

as counterstain, 20

as double stain, 35

Eosinophile granules, 28

leucocytes, 28, 73-77

Ermengem, Van, 38

Erysipelas, 51

Examination of air, 109-115 (vide Air)

anaërobic germs, 113-115

animal (anthrax), 27

(cholera), 44

dead of a bacterial disease, 132-135

antiseptics, 126

decomposing meat, 120-124

diseased meat, 120-124

disinfectant gases, 129.131

disinfectant solutions, 127

dust (vide Air)

filters (vide Filters)

fresh tissues (films), 27, 28

frozen sections, 35

gelatine plates, 34,44

ice cream, 124, 125

milk, 105-108

soil, 115, 118

stained films, 9-10

tubercular sputum, 59-61

water, 93-104

Ferment, 161-166 (vide Enzyme)

action of chloroform on, 162

bacterial, 169

curdling , 21, 45

Fermi, 168, 169

Fibrin, staining of, 51, 52

Films, preparation of, 8,19 
Films, preparation of, from anthrax tissues, 27, 28

Filter, Berkefeld, 98, 106, 125, 136, 141, 142

effect of use on, 137

testing of, 136

sugar, 110-112

Flagella, staining of, 38,39

Flasks, cleaning of, 82 sterilisation of, 83

\section{Fordos, 147}

Frozen sections, 35, 50-52, 62, 63 anthrax, 35

tubercle, 62,63

Fuchsine, aqueous solution, 9

aniline, 30

carbol, 31

Ehrlich's, 30

Löffler's, 13

Ziehl's, 31

Gelatine, carbol, 171

thymol, 169

plates, examination of, 34

preparation of nutrient, 86

sterilisation of, 87

to fill tubes with, 87

Gentian-violet, aqueous solution, 9 aniline water, 18

carbol, 61

Gessard, 147

Glanders, staining of paraffin sections, 68, 69

Glycerine broth, preparation of, 85

agar-agar, preparation of, 91

Gram's iodine solution, 18

Gram's method of staining, anthrax, 27

celloidin sections, 45,46

films, 17, 18, 19

frozen sections, 36,37

paraffin sections, 70,71

pus, 20

tubercle, 63

Grape-sugar agar-agar, preparation of, 91

to fill tubes with, 91
Grape-sugar broth, preparation of, 85

gelatine, preparation of, 87

Griess's reaction, 143

Gruber's method, 103

HangING drop cultures, 14, 17

examination of, 15

staining of, 17-19

phagocytosis in, $71,72,76,77$

Hardening of tissues, 133, 134

Hetero-albumoses, 152, 153, 154

ICE cream, examination of, 124,125

Immunity, experiments on frog, 73,74

Impression specimens-

gelatine plates, 34

sloped gelatine, 26

staining of, 26

"Intracellular" poisons, 142

Invertine, 161-168

action of chloroform on, 162

separation of, 164

test for, 165

Iodine, Gram's solution, 18

Weigert's solution, 52

\section{KLEIN, 147}

LEPROSY bacillus, staining of frozen sections, 57

Leucocytes, staining of, 28 eosinophile, 73-77

Madura disease, 46

Malignant œdema, 116-118

Mammalian tubercle, 67

Martin, 155

Meat infusion, preparation of, 86

Metabolic products of bacteria, 141 (vide Toxines, Albumoses, Proteïnes, Ptomaines)

Method, Gram's, 17

Gruber's (cholera), 103

peptone (cholera), 101, 102

Van Ermengem's (Hagella), 33 
Method, Van Ketel's, 61, 107 Weigert's, 51, 52, 66, 69

Methylene-blue, aqueous solution, 9 and eosine, 28,36 and picrocarmine, 36

Micrococci : pneumoniæ (see Pneumonia)

pyogenic, 50-52

Staphylococcus cereus flavus, 7, 47,48

Staphylococcus pyogenes albus, 47,48

Staphylococcus pyogenes aureus, $7,8,47,48$

Streptococcus erysipelatos (vide Streptococcus)

Streptccoccus pyogenes (vide Streptococcus)

tetragonus, 51

Milk tubes, preparation of, 89 sterilisation of, 89

Mycetoma, 46

Mycoproteïne, 145

NeNCKI, 144

Nitrous acid in cultures, 142

Nutrient media: carbol gelatine, 171

Fernii's solution, 170

preparation of, $83-92$

thymol gelatine, 169

OXYaEN, action on bacteria, 5

PARAFFin sections, 64

artinomycosis, 69,70

diphtheritic membrane, 65,66

glanders, 68,69

pyæmic spleen, 70,71

tubercle, 64,65

embedding, 133, 135

Peptone method (cholera), 101, 102

Peptones, 150

tests for, 150

Peptone solution, preparation of, 88 sterilisation of, 89

Phagocytes, 72-77 staining of, 72,73
Phagocytosis, 71, 77

effect of anæsthesia on, 76

effect of heat on, 74-76

examination in hanging drop, 71 , $72,76,77$

examination in stained specimens, 72,73

Picrocarmine, 36

Plates, agar-agar, 103, 104

gelatine, 29

anthrax, 34

cholera, 42, 44, 104

quantitative examination of water, 93-95

air and dust, 109

Pneumococcus (vide Pneumonia)

Pneumonia, cultivations, 47

coccus of, 50

fibrin staining, 51,52

tissues, 51, 52

Pneumonic sputum, staining of, 56 double staining of, 56

Poisons, bacterial, 141, 144, 155, $158,161,167,172$

intracellular, 142

Potato tubes, 89 sterilisation of, 89

Preparation of nutrient media, 83-92 serum tubes, 155

Products, bacterial, 141, 144, 147, $155,158,161,167,172$

Proteïnes, 144-146

Proteolytic ferments and enzymes, 168-171

Proto-albumose (vide Albumoses)

Psorosperms, 124

Ptomaines, 172-174

Pus, staining of, 19, 20, 48, 49 with methylene-blue, 20

by Gram's method, 20

Putrefaction, 120, 122

Putrescine, 172-174

hydrochlorate of, 174

Pyæmic abscess, 51

spleen, 51, 70, 71

Pyocyanine, 147

Pyogenic cocci, 47, 50, 52

Pyrogallic acid, 114 
QUinine, sulphate of, 160

Rolu tubes, 95, 96, 114

Sarcina lutea, 6,8 staining of, 10, 11

Sections, frozen, $35-37,51,57,62$ (vide Staining)

actinomycosis, 45,69

anthrax, 35, 37

celloidin, 45,46

diphtheria, 57, 65, 66

erysipelas, 51

glanders, 68

leprosy, 57

Madura disease, 46

micrococcus tetragonus, 51

mycetoma, 46

paraffin (vide Paraffin), 64

pneumonia, 51, 52

pyæmia, 51,70

tubercle, $62-65$

typhoid spleen, 55

Separation of hay bacillus, 33

Serum tubes, preparation of, 155

Shake cultures, 99

Silk threads, anthrax, 127

Soil, examination of, 115-118

for malignant œedema bacilli, 116

for tetanus bacilli, 116

Spores, tetanus, 69,118

staining of, $30-32$

Sputum, pneumonic, 56

tubercular, 59, 61

carbolised, 61

fresh, 59

watery, 62

Staining methods, Gram's, 17-19, 20

Van Ermengem's, 38, 39

Weigert's fibrin staining, 51, 52

Van Ketel's, 61, 107

Staining of anthrax tissues, 27, 28

celloidin sections, $35-37$

flagella, 38,39

frozen sections, 35,37

hanging drop cultures, 17-19

impression specimens, 23
Staining of micro-organisms with simple aniline dyes, 9-11

paraffin sections, 64-66, 68-71

psorosperms, 124

pus, 19,20

sputum (tubercle), 59-62

Staining solutions, aniline fuchsine, 30

aniline gentian-violet, 18

carbol fuchsine, 31

gentian-violet, 61

methylene-blue, 68

Czinzinski's, 35, 36

Ehrlich's fuchsine, 30

eosine, $28,71,72$

eosine and methylene-blue, 36

for flagella staining, 38

Gram's method, 18

pneumococcus capsule, 55

spore staining, 30,32

Weigert's method, 52

fuchsine, 9

gentian-violet, 9

Löfller's fuchsine, 13

methylene-blue, 9

preparation of simple, 9

Ziehl's fuchsine, 31

Staining, double, with methyleneblue and eosine, 28,35

with methylene-blue and picrocarmine, 36

Staphylococcus cereus flavus, 8, 47

Staphylococcus pyogenes aureus, $8,19,47,48$

albus, 47

Sterilisation and putrefaction, 121, 122

Sterilisation by heat, 141

by filtration, 141

of beakers, 83

flasks, 83

pipette, 93, 94

test-tubes, 83

Streptococcus erysipelatos, 47

cultivations, 47

in hanging drop, 48

in tissues, 51 
Streptococcus pneumoniæ (vide Pneumonia)

pyogenes, $8,14,17$

cultures, 47

Gram's staining, 17

hanging drop, 50

in milk, 106

in sections, 51

Sugar filter, vide Filter

Sulphur dioxide, disinfectant action of, 129

Sunlight, action on bacteria, 4 cholera, 43

water, 97

TANk water examination, 96

Tap water, examination of, 93-96

Temperature, action of bacteria, 3

Testing of filters, 136-138

Test-tubes, cleaning of new, 81

used, 82

sterilisation, 83

Tetanus bacillus, 69

fractional separation of, 118

growth in exhausted flask, 116

growth in hydrogen, 116

separation from soil, 116-118

staining of, 69

spores, 69

Torula alba, 6, 8, 13

Toxine, diphtheria, 158, 159

Trichina spiralis, 123

Tryptic enzymes, 168-171

ferments, $168-171$

Tubercle, avian, 67

bacilli, 67

frozen sections, 62,63

Gram's method, 63

mammalian, 67

milk, 107

paraffin sections, 64,65

sputum, fresh, 59

carbolised, 61

watery, 62

urine, 62

Van Ketel's method, 61, 107

Typhoid, action of carbolic acid on, 126
Typhoid bacilli, 38, 39, 53, 54, 59

cultivations, 53

examination of water, 98-100

flagella, 38, 39

in milk, 106

in spleen, 55

in water, 98

shake cultures, 54,99

staining of, 54

25 per cent gelatine, 99

UDRANSKY, 172

Ulcerative endocarditis, 51

Urine, examination for tubercle bacilli, 62

Uschinsky, 158

Van Ermengem, 38, 39

Van Ketel, 61, 107

Vibrio choleræ Asiaticæ (vide Cholera)

Finkler-Prior, 37, 40, 168

Metchincovi, 37, 40

WATER, 93-104

bacillus of typhoid fever, $98-100$

bacterium coli commune, 98-100

cholera, 101-104

effect of sunlight on, 97

filtration, 136-138

qualitative examination, 98-104

quantitative examination of, $93-97$ distilled, 96

tank, 96

tap, 93-98

Weigert's iodine solution, 52

Weigert's method, actinomycosis, 69,70

croupous pneumonia, 51,52

diphtheritic membrane, 66

fibrin staining, $51,52,66,69,70$

YEAST, 161-167

enzyme, 167, 168

ferment, 161,168

invertine, 161,168 
Printed by R. \& R. CLARK, Edinburgh. 


\section{MACMILLAN AND CO.'S PUBLICATIONS.}

\section{BY E. KLEIN, M.D.}

THE BACTERIA IN ASIATIC CHOLERA. By E. Klein, M.D., F.R.S., Lecturer on General Anatomy and Physiology in the Medical School of St. Bartholomew's Hospital, Professor of Bacteriology at the College of State Medicine, London. Crown 8vo. 5s.

MICRO-ORGANISMS AND DISEASE : an introduction into the study of specific micro-organisms. With ro8 Engravings. Second Edition. Crown 8vo. 6s.

THE ETIOLOGY AND PATHOLOGY OF GROUSE DISEASE, FOWL ENTERITIS, AND SOME OTHER DISEASES AFFECTING BIRDS. With 6o Illustrations. $8 \mathrm{vo}$. 7 s. net.

METHODS OF PATHOLOGICAL HISTOLOGY. By C. VoN KAHLDEN, Assistant Professor in the University of Freiburg. Translated and Edited by H. Morley Fletcher, M.A., M.D. With an Introduction by G. Sims Woodhead, M.D. 8 vo. $6 \mathrm{~s}$.

$$
\text { ** A Companion Volume to Ziegler's "Pathological Anatomy." }
$$

AERO-THERAPEUTICS : OR, TIIE TREATMENT OF LUNG DISEASE BY CLIMATE. Being the Lumleian Lectures for $\mathrm{r} 893$, delivered before the Royal College of Physicians. With an Address on the High Altitudes of Colorado. By Charles Theodore Williams, M.A., M.D., Oxon., F.R.C.P. Senior Physician to the Hospital for Consumption and Diseases of the Chest, Brompton, late President of the Royal Meteorological Society. 8vo. 6s. net.

MATERIALS FOR THE STUDY OF VARIATION. Treated with Especial Regard to Discontinuity in the Origin of Species. By Wrlliam Bateson, M.A. Fellow of St. John's College, Cambridge. 8vo. 2Is, net.

PRACTICAL BOTANY FOR BEGINNERS. By F. O. BOWER, D.Sc., F.R.S., Regius Professor of Botany in the University of Glasgow, Author of "A Course of Practical Instruction in Botany." Globe 8vo. 3s. 6d.

THE STUDY OF THE BIOLOGY OF FERNS BY THE COLLODION METHOD. For Advanced and Collegiate Students. By George F. Atrinson, Ph.B., Associate Professor of Cryptogamic Botany in Cornell University. 8vo. 8s. 6 d. net.

$$
\text { MACMILLAN AND CO., LONDON. }
$$




\section{MACMILLAN AND CO.'S PUBLICATIONS.}

A TEXT-BOOK OF PATHOLOGY : SYSTEMATIC AND PRACTICAL. By Professor D. J. Hamilton. Copiously Illustrated. Vol. II., Parts I. and II. Medium 8vo. I5s. net each Part. (Vol. I. 21S. net.)

A TEXT-BOOK OF COMPARATIVE ANATOMY. By Dr. Arnold Lang, Professor of Zoology in the University of Zurich. With Preface to the English Translation by Dr. ERnst HaEckel. Translated by Henry M. Bernard, M.A., Cantab., and Matildd Bernard. Vol. I. 8vo. i7s. net.

[Vol. II. in the Press.

LESSONS IN ELEMENTARY ANATOMY. By St. George Mivart, F.R.S., Author of "The Genesis of Species." Fcap. 8vo. 6s. 6d.

ELEMENTS OF THE COMPARATIVE ANATOMY OF VERTEBRATES. Adapted from the German of RoBert Wiedersheim, Professor of Anatomy, and Director of the Institute of Human and Comparative Anatomy in the University of Freiburg, in Baden. By W. Newton PARker, Professor of Biology in the University College of South Wales and Monmouthshire. With additions by the Author and Translator. 270 Woodcuts. Medium 8vo. 12s. 6d.

A COURSE OF INSTRUCTION IN ZOOTOMY. Vertebrata. By T. JefFery PARker, F.R.S., Professor of Biology in the University of Otago, N.Z. With Illustrations. Crown 8vo. 8s. 6d.

AN INTRODUCTION TO THE OSTEOLOGY OF THE MAMMALIA. By Sir William HenRy Flower, F.R.S., F.R.C.S., Director of the Natural History Department of the British Museum. Illustrated. Third Edition. Revised with the assistance of Hans Gadow, Ph.D., Lecturer on the Advanced Morphology of Vertebrates in the University of Cambridge. Crown 8vo. Ios. 6d.

THE MYOLOGY OF THE RAVEN (Corvus corax Sinuatus). A Guide to the Study of the Muscular System in Birds. By R. W. ShureldT, of the Smithsonian Institute, Washington, U.S.A. With Illustrations. 8 vo. I3s. net.

A COURSE OF ELEMENTARY PRACTICAL HISTOLOGY. By William Fearnley. Crown 8vo. 7s. 6d.

A COURSE OF ELEMENTARY PRACTICAL PHYSIOLOGY AND Histology. By Prof. Michael Foster, M.D., F.R.S., and J. N. LANGLey, F.R.S., Fellow of Trinity College, Cambridge. Sixth Edition. Crown 8vo. 7s. 6d.

LESSONS IN ELEMENTARY PHYSIOLOGY. By T. H. HUXLEY, F.R.S. With numerous Illustrations. Fourth Edition. Pot 8vo. 4s. 6d.

QUESTIONS ON HUXLEY'S LESSONS IN ELEMENTARY PHYSIOLOGY. For the Use of Schools. By Thomas Alcock, M.D. Fifth Edition. Pot 8vo. Is. 6d. 

UNIVERSITY OF CALIFORNIA MEDICAL SCHOOL LIBRARY

THIS BOOK IS DUE ON THE LAST DATE STAMPED BELOW

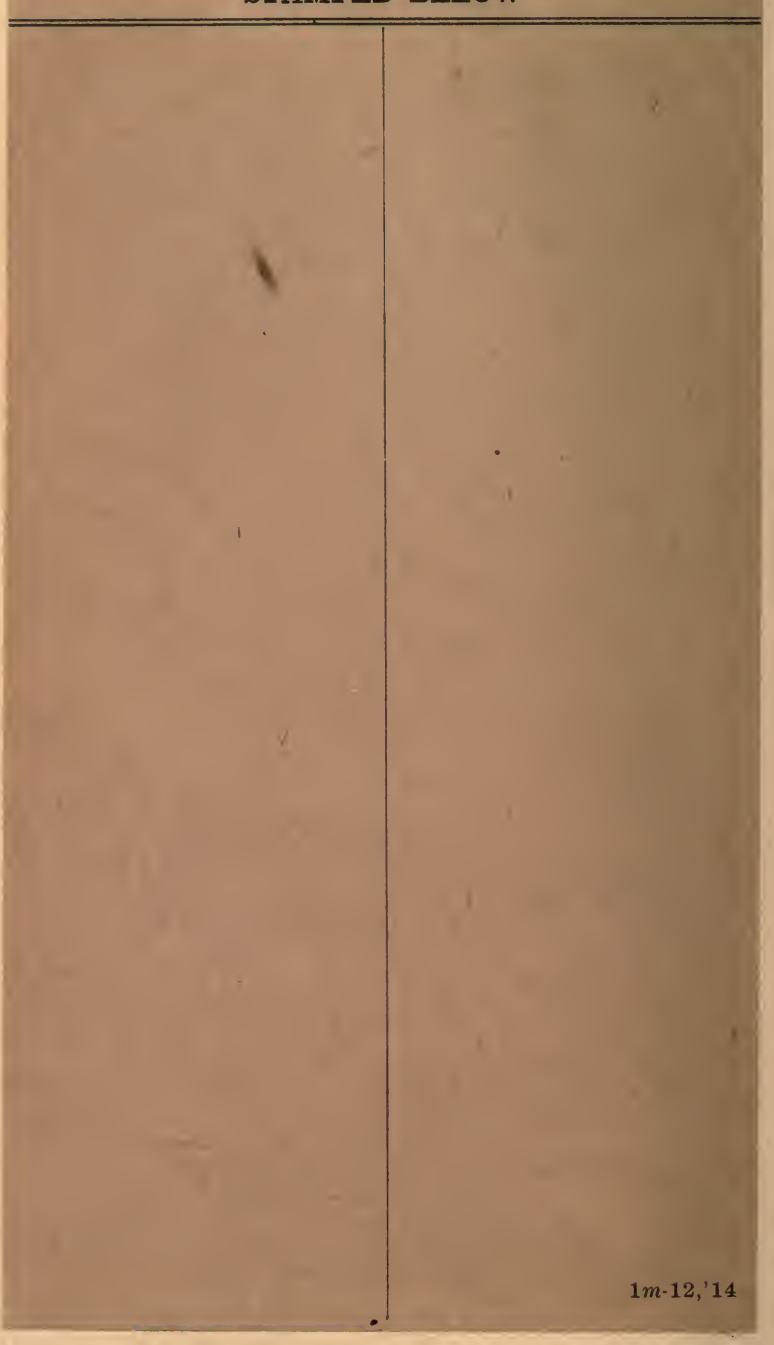


LeS Kanthack, A.A. 423

KI6 A course of elementary 1895 practical bacteriologJ by A.A.Kanthack and J.H.Drysale. 


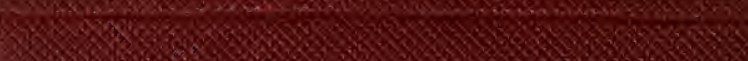

$3 \times 2 \times 5$

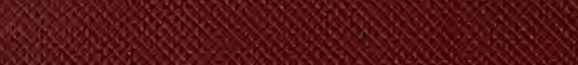

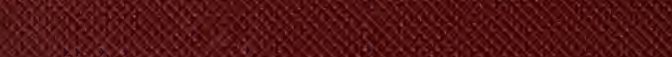

2.

2000

$8000 \%$.

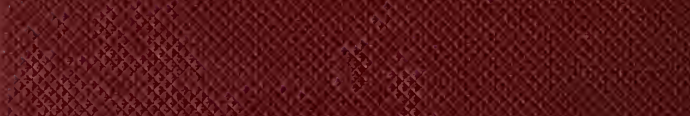

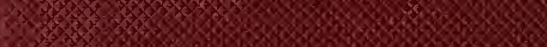

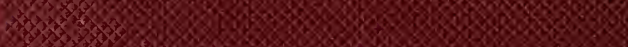

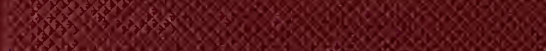

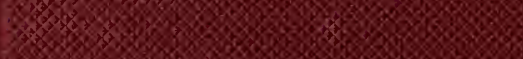

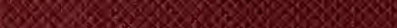

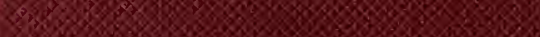

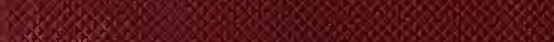

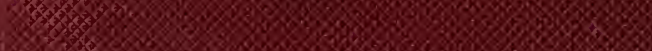

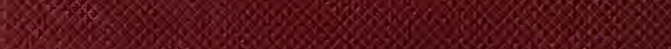

20083

tors 2008

$00 \% 30$

300638

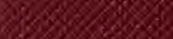

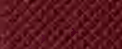

3028

10980

$3083 \%$

65003835

$3 \%$

$398 \% 8$

3898968

38989

3098 